\title{
Impact of size-dependent grain temperature on gas-grain chemistry in protoplanetary disks: The case of low-mass star disks
}

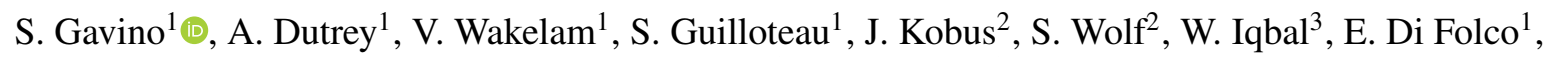 \\ E. Chapillon ${ }^{1,4}$, and V. Piétu ${ }^{4}$
}

\author{
${ }^{1}$ Laboratoire d'Astrophysique de Bordeaux, Université de Bordeaux, CNRS, B18N, Allée Geoffroy Saint-Hilaire, 33615 Pessac, \\ France \\ e-mail: sacha.gavino@nbi.ku.dk \\ ${ }^{2}$ University of Kiel, Institute of Theoretical Physics and Astrophysics, Leibnizstrasse 15, 24118 Kiel, Germany \\ ${ }^{3}$ South-Western Institute for Astronomy Research (SWIFAR), Yunnan University (YNU), Kunming 650500, PR China \\ ${ }^{4}$ IRAM, 300 rue de la piscine, 38406 Saint Martin d'Hères Cedex, France
}

Received 29 June 2020 / Accepted 28 May 2021

\begin{abstract}
Context. Grain surface chemistry is fundamental to the composition of protoplanetary disks around young stars.

Aims. The temperature of grains depends on their size. We evaluate the impact of this temperature dependence on the disk chemistry. Methods. We modeled a moderately massive disk with 16 different grain sizes. We used the 3D Monte Carlo POLARIS code to calculate the dust grain temperatures and the local $u v$ flux. We modeled the chemistry using the three-phase astrochemical code NAUTILUS. Photo processes were handled using frequency-dependent cross sections and a new method to account for self and mutual shielding. The multi-grain model outputs are compared to those of single-grain size models $(0.1 \mu \mathrm{m})$; there are two different assumptions for their equivalent temperature.

Results. We find that the Langmuir-Hinshelwood mechanism at equilibrium temperature is not efficient to form $\mathrm{H}_{2}$ at 3-4 scale heights $(H)$, and we adopt a parametric fit to a stochastic method to model $\mathrm{H}_{2}$ formation instead. We find the molecular layer composition $(1-3 H)$ to depend on the amount of remaining $\mathrm{H}$ atoms. Differences in molecular surface densities between single and multi-grain models are mostly due to what occurs above $1.5 \mathrm{H}$. At 100 au, models with colder grains produce $\mathrm{H}_{2} \mathrm{O}_{\text {and }} \mathrm{CH}_{4}$ ices in the midplane, and those with warmer grains produce more $\mathrm{CO}_{2}$ ices; both of these allow for an efficient depletion of $\mathrm{C}$ and $\mathrm{O}$ as soon as $\mathrm{CO}$ sticks on grain surfaces. Complex organic molecules production is enhanced by the presence of warmer grains in the multi-grain models. Using a single-grain model mimicking grain growth and dust settling fails to reproduce the complexity of gas-grain chemistry.

Conclusions. Chemical models with a single-grain size are sensitive to the adopted grain temperature and cannot account for all expected effects. A spatial spread of the snowlines is expected to result from the ranges in grain temperature. The amplitude of the effects depends on the dust disk mass.
\end{abstract}

Key words. circumstellar matter - protoplanetary disks - astrochemistry - stars: pre-main sequence - radio lines: stars radiative transfer

\section{Introduction}

As planets form in protoplanetary disks, studying the physical and chemical evolution of their gas and dust content along the whole disk lifetime is important in order to investigate how planetary formation proceeds. In the early stages of disks (the so-called protostellar phase) small dust grains are essentially dynamically well coupled to the gas. When grain growth occurs (up to at least centimeter-sized particles) larger particles, which dynamically decouple from the gas phase, settle toward the disk midplane. This vertically changes the gas-to-dust ratio in the disk. Moreover, grain growth also allows $u v$ to penetrate deeper in the disk, changing its chemistry.

As a consequence of the complex interplay of physical and chemical processes, it is now demonstrated that the disk structure can be separated vertically in three distinct regions: from top to bottom, the irradiated tenuous disk atmosphere (above $3-$ $4 H$ or scale heights), where the gas can be hot and is ionized or in atomic form; the warm denser molecular layer (1-3 $H)$, where most molecules reside; and below $1 H$, the midplane. Beyond the $\mathrm{CO}$ snowline, which is typically located at a radius about $20 \mathrm{au}$ in a T Tauri disk, the midplane is very dense and cold $(\leq 20 \mathrm{~K})$. This area is essentially shielded from the stellar radiation, with low ionization and turbulence levels. In this cold region, most molecules are stuck onto dust grains, which exhibit icy mantles that can still be processed by cosmic rays.

Thanks to The Atacama Large Millimeter/submillimeter Array (ALMA), several high angular resolution and sensitive observations of disks orbiting either low- or intermediate-mass young stars ( $\mathrm{T}$ Tauri and Herbig Ae stars) have quantitatively confirmed this general scheme. CO observations of HD 163296 have clearly shown the $\mathrm{CO}$ gas-phase depletion in the dark, cold disk midplane (de Gregorio-Monsalvo et al. 2013), also revealing that the CO gas is located in the intermediate warm molecular layer. More recently, the ALMA observations of the edge-on T Tauri disk called the Flying Saucer (Dutrey et al. 2017) directly show the molecular layer at intermediate scales $(1-2 H)$ with $\mathrm{CS}$; the denser gas tracer is located at about $1 \mathrm{H}$ below the $\mathrm{CO}$ 
emission (1-3 H), which better traces the whole envelope of the molecular layer.

In the last ten years, several astrochemical models have emerged that attempt to incorporate the observed complexity of disk physics to provide more accurate molecular abundance and surface density predictions. While the very first models included only the gas-phase chemistry, gas-grain coupling was added to take into account firstly adsorption and desorption for a few molecules (e.g., Woitke et al. 2009) and then reactions on the grain surface (e.g., Semenov \& Wiebe 2011; Walsh et al. 2014). Another step was finally achieved with models. This step includes three material phases: the gas-phase and two phases for the gas-grain chemistry with grains modeled by an icy surface layer and an active mantle (Ruaud \& Gorti 2019; Wakelam et al. 2019). Thermochemical models that calculate the density and thermal structures in a self-consistent manner have also been developed (e.g. Hollenbach \& Gorti 2009). Recently, Ruaud \& Gorti (2019) have also coupled such a thermochemical model (Gorti et al. 2011) to a three-phase chemical model.

An important improvement in chemical models was to introduce more realistic dust disk structures by taking into account grain growth and dust settling. Grain growth reduces the dust cross section and therefore increases the $u v$ penetration, while dust settling locally changes the gas-to-dust ratio. Grain growth was first introduced in disk chemistry by Aikawa \& Nomura (2006). Dust settling is often mimicked by adding larger grains in the midplane (e.g., Wakelam et al. 2019).

Several authors have already studied the effect of multiple grain sizes on chemistry. As an example, Acharyya et al. (2011) used five different grain sizes in their models but the grain temperature was the same for all grains. Following this work, Pauly \& Garrod (2016) tested the impact of a simple temperature distribution, where $T(a) \propto a^{-(1 / 6)}, a$ being the grain size, while Ge et al. (2016) explored a small range of temperatures (14.9-17.9) for the cold neutral medium. More recently, Iqbal \& Wakelam (2018) also developed a model handling different grain sizes.

On another hand, Akimkin et al. (2013) developed a disk model in which they couple the dust grain time evolution (grain growth and settling) to chemistry. Another improvement was achieved with the development of grids of thermochemical models allowing multiwavelength fitting of dust and molecular lines such as DIANA (Woitke et al. 2019). However, these elaborate approaches use a single-grain temperature that is either fixed or derived from a dust radiative transfer simulation, while the temperature of a grain depends on its size (e.g., Wolf 2003), larger grains being colder. Chapillon et al. (2008) found an important CO depletion in the disk of CQ Tau, whose temperature is well above the $\mathrm{CO}$ snowline temperature. These authors suggested that $\mathrm{CO}$ may remain trapped onto larger grains that are cold enough to prevent thermal $\mathrm{CO}$ desorption. Heese et al. (2017) investigated the dust temperature variations with grain size and position (radius and altitude above the midplane) in a typical $\mathrm{T}$ Tauri disk using the 3D dust radiative transfer code Mol3D (Ober et al. 2015). The dust temperature variations in the molecular layer appear to be significant enough to affect the disk chemistry. This dependence questions the applicability of elaborate, self-consistent, and thermochemical models that rely on a single-grain size and temperature.

We explore in this work the impact of the variations of the grain temperature with their size onto the chemistry of a representative T Tauri disk. Our goal is not to build a complete chemical model that incorporates the best of all previous studies, neither to make grid models that could provide relevant comparisons with the observations. Instead, we only intend to check the limits of an assumption that is used in most previous models, and we explore the main consequences of dust grain temperature dependence with size.

For this purpose, we coupled the 3D Monte Carlo continuum radiative transfer code POLARIS (Reissl et al. 2016) to the NAUTILUS Multi Grain Code (Hersant et al. 2009; Ruaud et al. 2016) using the three-phase version, which also takes into account a grain size distribution (Iqbal \& Wakelam 2018). We present the two codes and how we parameterize the gas and dust disk in Sect. 2. Section 3 deals with the disk model description and global results. We discuss in more detail the results in Sect. 4 and we state our conclusions in Sect. 5.

\section{Model description}

To estimate the impact of the grain temperature onto the chemistry, we couple the radiative 3D Monte Carlo code POLARIS with the astrochemistry code NAUTILUS. This requires that we independently assume a gas and dust disk structure, a gas temperature, a dust distribution, and settling. Moreover, the coupling between the two codes requires a new method to calculate the dust extinction for NAUTILUS, the self and mutual shielding using the $u v$ flux calculated by POLARIS in each disk cell. Figure 1 is a scheme summarizing how we proceed.

\subsection{Disk model}

Our disk structure is derived from a simple disk model in which a truncated power laws describe most of the quantities: surface density, midplane temperature, velocity field, with sharp inner and outer radii. A detailed model description is given in Appendix A.

We adopt a power-law grain size distribution $\mathrm{d} n(a)=C a^{-d} \mathrm{~d} a$ with $\mathrm{d} n(a)$, the number of grains of size $a$, and $C$ a normalization constant that can be derived from the dust mass (see Appendix A.2). The resulting distribution is discretized into 16 logarithmically distributed intervals that have grain sizes ranging from $a_{\min }=5 \mathrm{~nm}$ to $a_{\max }=1 \mathrm{~mm}$. The fraction of mass and area per bin are given in Table A.1.

Because our goal is to evaluate the impact of dust grain temperatures, we assume a common gas temperature structure for all our simulations (including single and multi-grain models) to avoid chemical effects that could be due to gas alone. We selected values derived from the observations of the Flying Saucer T Tauri disk (see Dutrey et al. 2017), whose edge-on orientation facilitates a direct measure of the 2D gas temperature distribution from ALMA observations of the optically thick CO $J=2-1$ line. Values are given in Table 1 .

The vertical distribution of gas is self-consistently derived from the imposed temperature law. However, to account for dust settling, we assume grains of a given size follow a simple Gaussian vertical profile, whose scale height is simply related to the gas hydrostatic scale at the disk midplane temperature according to a simple prescription (see Appendix A.2). Our model is therefore not fully consistent since very small grains should follow the vertical profile of the gas, but should be adequate for the larger grains observed in disks. To compute the settling, we assume an $\alpha$ viscosity of 0.01 and a Schmidt number of $S_{\mathrm{c}}=1$. Although often assumed in disk modeling, these values are somewhat arbitrary and observations with ALMA are not yet accurate enough to allow for a quantitative description of dust settling.

Figure 2 is a 2D representation of the disk structure in density and in temperature for the gas and for the dust (multi-grain model with settling). 


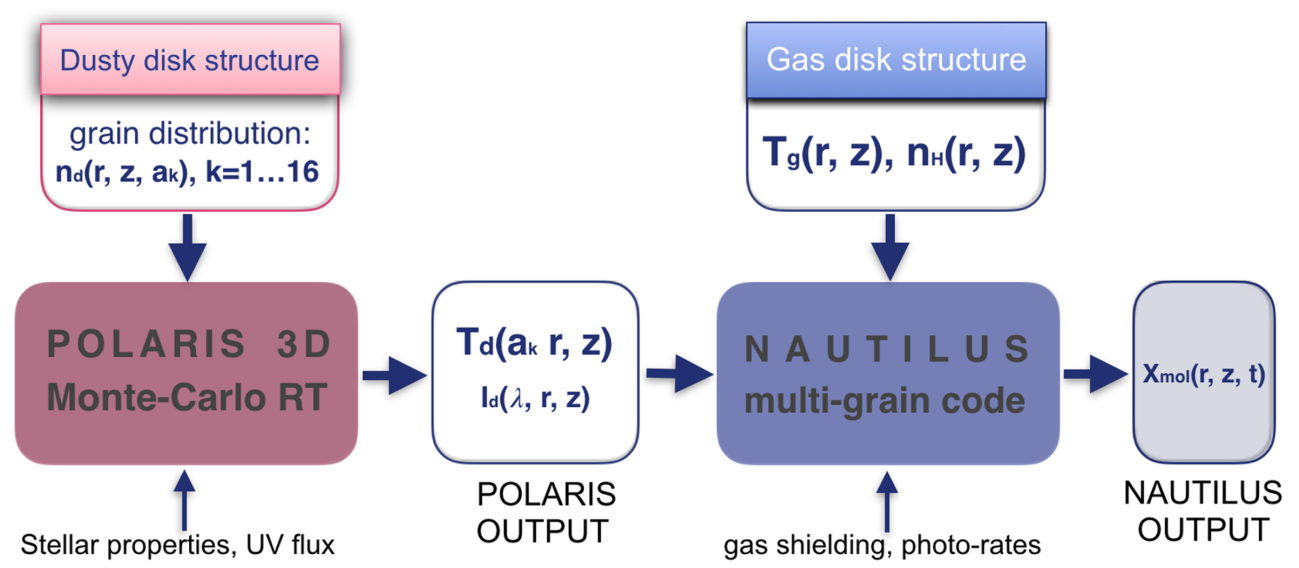

Fig. 1. Multi-grain model: coupling scheme between NAUTILUS and POLARIS.

Table 1. Overview of the disk model parameters.

\begin{tabular}{|c|c|}
\hline \multicolumn{2}{|l|}{ Disk parameters } \\
\hline$T_{\star \text {,eff }}($ star temperature $)$ & $3900 \mathrm{~K}$ \\
\hline$L_{\star}^{\star}($ star luminosity $)$ & $0.75 L_{\odot}$ \\
\hline$M_{\star}($ star mass $)$ & $0.58 M_{\odot}$ \\
\hline ISRF & DRAINE \\
\hline $\boldsymbol{\Sigma}_{\mathrm{g}, 0}\left(\right.$ gas $\left(\mathrm{H}+\mathrm{H}_{2}\right)$ surface density at $\left.R_{0}\right)$ & $0.335 \mathrm{~g} \mathrm{~cm}^{-2}$ \\
\hline $\boldsymbol{p}$ (surface density exponent) & 1.5 \\
\hline$R_{\text {in }}$ (innermost radius) & $1 \mathrm{au}$ \\
\hline$R_{\text {out }}$ (outermost radius) & $250 \mathrm{au}$ \\
\hline$R_{0}$ (reference radius) & $100 \mathrm{au}$ \\
\hline$\zeta$ (dust to gas mass ratio) & 0.01 \\
\hline$T_{\text {mid, } 0}$ (observed $T_{\text {mid }}$ at $\left.R_{0}\right)$ & $10 \mathrm{~K}$ \\
\hline$T_{\mathrm{atm}, 0}\left(\right.$ observed $T_{\mathrm{atm}}$ at $\left.R_{0}\right)$ & $50 \mathrm{~K}$ \\
\hline$\sigma$ (stiffness of the vertical $T$ profile) & 2 \\
\hline$q$ (radial $T$ profile exponent) & 0.4 \\
\hline$S_{\mathrm{c}}$ (Schmidt number) & 1 \\
\hline$\alpha$ (viscosity coefficient) & 0.01 \\
\hline$d$ (grain size distribution exponent) & 3.5 \\
\hline$a_{\min }$ (smallest grain radius) & $5 \mathrm{~nm}$ \\
\hline$a_{\max }$ (biggest grain radius) & $1 \mathrm{~mm}$ \\
\hline Number of bins & 16 \\
\hline$\rho_{\text {grain }}$ (material density of dust) & $2.5 \mathrm{~g} \mathrm{~cm}^{-3}$ \\
\hline$M_{\text {disk }}($ total disk mass $)$ & $7.5 \times 10^{-3} M_{\odot}$ \\
\hline
\end{tabular}

\subsection{Radiation fields and dust temperature}

In this new approach, we use the dust radiative transfer code to calculate the $u v$ field and dust temperature for each of the 16 grain sizes, in each cell of the disk (in radius and altitude), throughout the disk. The dust temperatures are then used to compute the chemistry with NAUTILUS.

However, the $u v$ field derived above only accounts for dust extinction and scattering. A proper accounting for self and mutual shielding of molecules and atoms is required to handle the photo-chemical processes.

\subsubsection{Radiation sources}

As the central heating source we assume a low-mass, pre-mainsequence star with a mass of $M_{\star}=0.58 M_{\odot}$, which radiates as a blackbody with an effective temperature of $T_{\star \text {,eff }}=3900 \mathrm{~K}$ and a luminosity $L_{\star}=0.75 L_{\odot}$. Additionally, we consider the interstellar radiation field (ISRF) with a spectral energy distribution (SED) from Draine (1978) between $91.2 \mathrm{~nm}$ and $200 \mathrm{~nm}$ and the extension of van Dishoeck \& Black (1982) for longer wavelengths. Besides the stellar radiation field, T Tauri stars exhibit a significant $u v$ excess coming from the inner disk boundary and accretion shocks on the stellar surface. This contribution is highly wavelength-dependent; spectral lines such as Lyman $\alpha$ have substantial intensities (France et al. 2014). As concerns the thermal balance of dust, the frequency dependence is largely irrelevant and can be absorbed by ensuring that the luminosity used in the model contains the $u v$ excess contribution. However, because photo processes are wavelength dependent, the details of the $u v$ spectrum shape strongly affect the chemistry. For this reason, we take as stellar input spectrum for our radiative transfer and chemical model the sum of the stellar blackbody spectrum and the $u v$ excess typically found in T Tauri stars. Further details about the radiation fields are given in Sect. 3.1.

\subsubsection{Radiative transfer simulations}

The radiation field is computed using the 3D Monte Carlo continuum and line radiative transfer code POLARIS (Reissl et al. 2016). As in Heese et al. (2017), we calculate the dust temperature distribution of 16 grain size intervals (see Fig. 3). We assume spherical grains with a size independent composition consisting of a homogeneous mixture of $62.5 \%$ silicate and $37.5 \%$ graphite. Optical properties from Draine \& Lee (1984) and Laor \& Draine (1993) are used to calculate the scattering and absorption coefficients based on Mie scattering (Mie 1908) using the MIEX code (Wolf \& Voshchinnikov 2004). The radiative transfer is solved using 100 wavelengths logarithmically spaced between $50 \mathrm{~nm}$ and $2 \mathrm{~mm}$. The spatial grid involves 300 radii, logarithmically spaced by a factor 1.05 between 1 and $300 \mathrm{au}$, and 181 angles with the same factor between 0 and $\pi$.

The temperature in the upper optically thin disk layers strongly depends on the grain size (Fig. 3, middle panel). The dust temperature increases with grain size for sizes in the range $0.007 \mu \mathrm{m}-0.070 \mu \mathrm{m}$, then decreases for sizes in the range $0.32-$ $15 \mu \mathrm{m}$, and increases again, although only slightly, with larger grain sizes. As explained in Heese et al. (2017), this can be understood by looking at the ratios of the absorption cross section for wavelengths at which the star radiates $(\approx 1 \mu \mathrm{m})$ and the absorption cross section for wavelengths at which the dust emits $(\approx 20 \mu \mathrm{m}$, Fig. 4). The ratio increases up to grain sizes of 

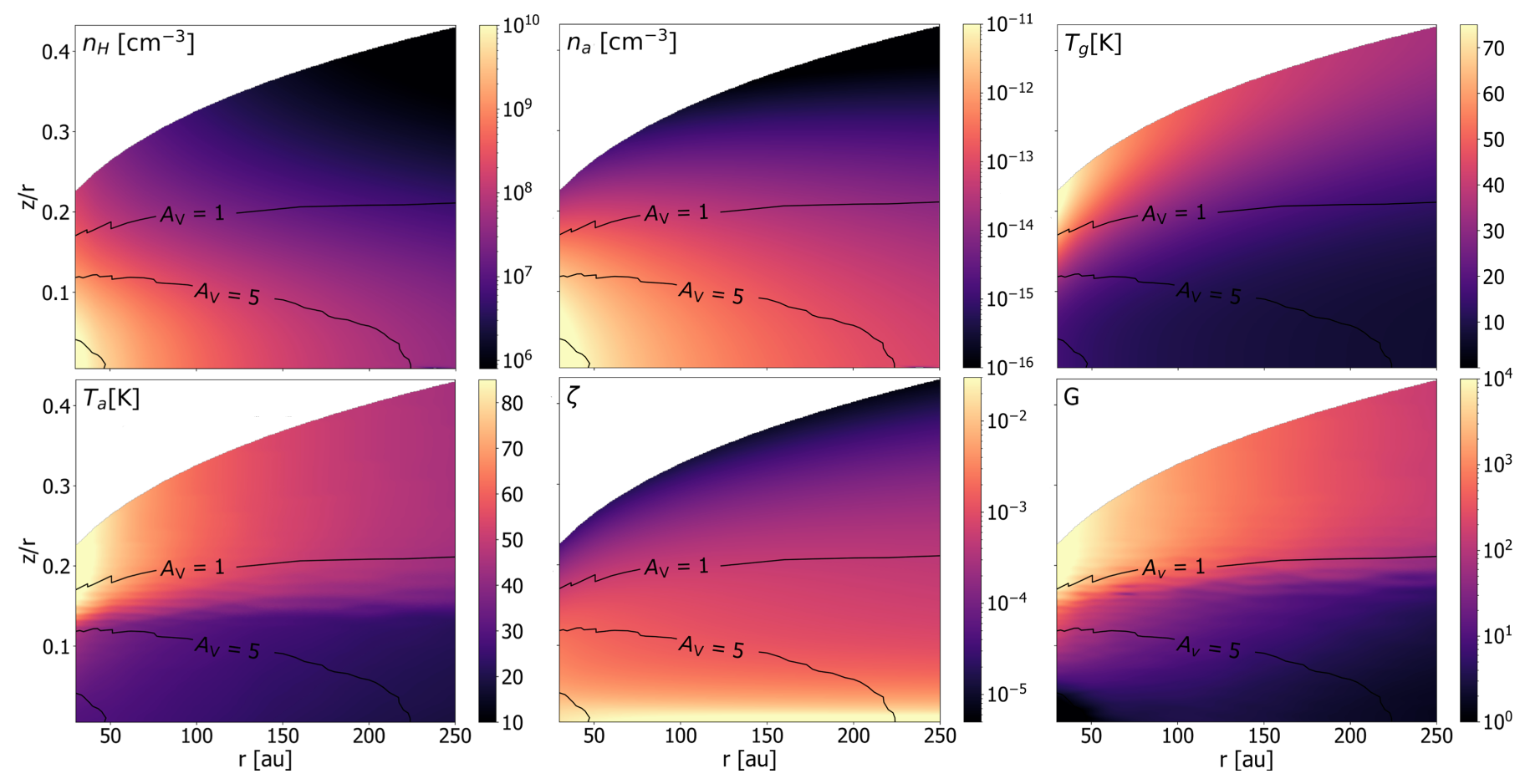

Fig. 2. Physical structure of the multi-grain models incorporating a full dust distribution. The quantity $T_{\mathrm{g}}$ is the gas temperature, $n_{\mathrm{H}}$ is the gas number density, $G$ is the local stellar and interstellar field in the case of the HUV models (see Appendix A), $\zeta$ is the dust to gas mass ratio, $T_{\text {a }}$ (Eq. (1)) is the area-weighted dust temperature, and $n_{\mathrm{a}}$ is the area-weighted grain number density. The quantity $A_{\mathrm{V}}$ is the visual extinction counted from disk surface toward the disk midplane.
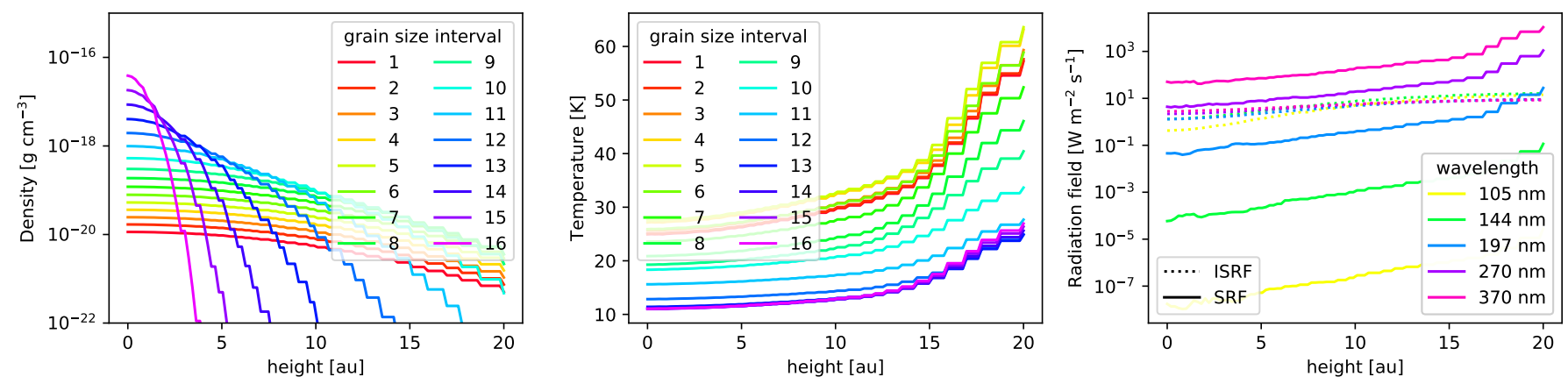

Fig. 3. Results of the radiative transfer simulation in vertical direction at a disk radius of $r=100$ au. Left: dust density distribution for the 16 grain size intervals as described in Appendices A.2.2 and A.2.3. Middle: dust temperature of the 16 grain size intervals. Right: stellar (solid lines) and ISRF (dotted lines).

$0.07 \mu \mathrm{m}$, meaning that the ratio of absorbed to emitted radiation increases, leading to higher grain temperatures. For larger grain sizes, the behavior is reversed until grains become larger than about $15 \mu \mathrm{m}$. Using these radiative transfer simulations we can obtain a distribution of dust temperatures $T_{\mathrm{d}}\left(a_{i}, r, z\right)$ that fully depend on the size and location of the grains. To compare our set of models with those consisting of a single-grain size (see Sect. 3.1.1), it is also convenient to introduce an area-weighted temperature defined as

$T_{\mathrm{a}}(r, z)=\frac{\sum_{i} a_{i}^{2} T_{\mathrm{d}}\left(a_{i}, r, z\right) n_{\mathrm{d}}\left(a_{i}, r, z\right)}{\sum_{i} a_{i}^{2} n_{\mathrm{d}}\left(a_{i}, r, z\right)}$.

We note that the area-weighted dust density $n_{\mathrm{a}}(r, z)$ (Fig. 2) is defined by an equation of the same form. Figure 5 shows the dust temperature profiles at 100 au used by all our different models.
Our disk model is less massive than that used by Heese et al. (2017), leading to smaller opacities, and thus the temperature differences between grains of different sizes is much larger in Figs. 3 and 5 than in that previous work.

\subsubsection{Dust extinction, self and mutual shielding}

The radiation field is a contribution of both the ISRF and the stellar radiation field (SRF). Radiation from these two sources encounters molecular or atomic gas and dust grains that affects its propagation through extinction and scattering. The effect of the dust is computed by the 3D Monte-Carlo continuum and line radiative transfer code POLARIS as described in Sect. 2.2.2 (Fig. 6 shows the sampling in the $90-400 \mathrm{~nm}$ wavelengths range relevant for photo processes). Figure 3 shows results at a disk radius of $r=100 \mathrm{au}$. 


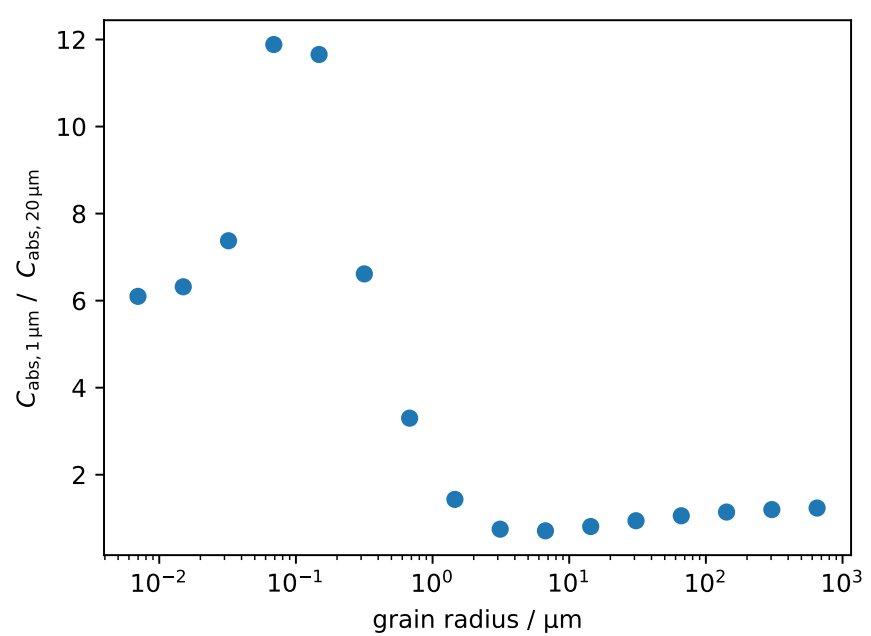

Fig. 4. Ratio of the absorption cross sections for wavelengths characteristic for stellar radiation $\left(C_{\mathrm{abs}, 1 \mu \mathrm{m}}\right)$ and dust emission $\left(C_{\mathrm{abs}, 20 \mu \mathrm{m}}\right)$.

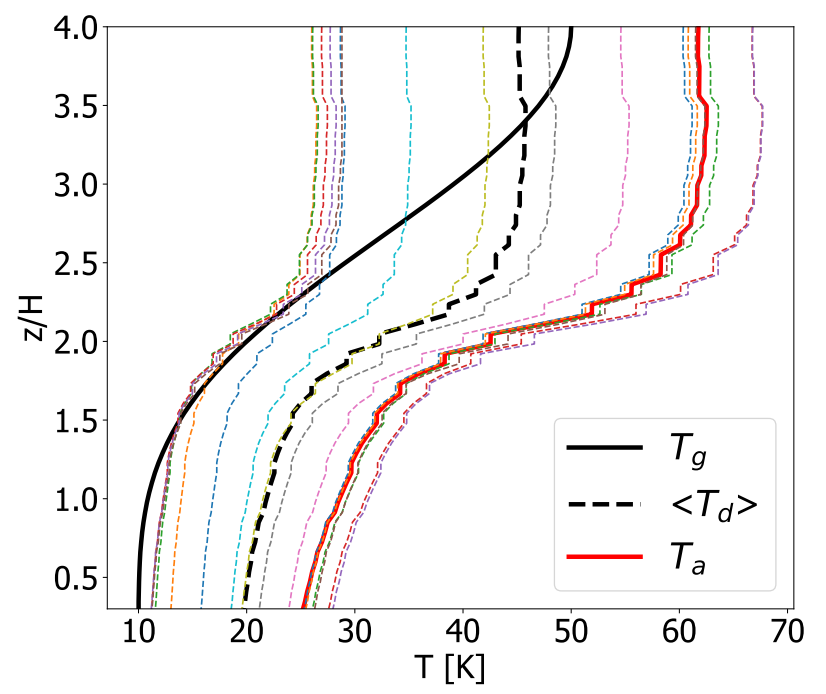

Fig. 5. Vertical profiles of temperatures at $100 \mathrm{au}$. The thick black line is the gas vertical temperature profile in Kelvin. The dotted colored lines are temperatures of each grain population and the thick red line is the area-weighted temperature $T_{\mathrm{a}}$. We see that temperatures are roughly constant for $z / H<1.5$ and for $z / H>2.5$. Between these two altitudes the temperatures exhibit a strong transition.

In chemical codes, the effect of extinction due to gas, known as self and mutual shielding, is often estimated using empirical attenuation factors as a function of line-of-sight visual extinction (e.g., Lee et al. 1996, for a discussion). The situation for disks is more complex owing to the two sources of $u v$ radiation (stellar neighborhood and ISRF) and varying dust properties. Empirical attenuation factors have been derived for disks (see, e.g., Visser et al. 2009; Heays et al. 2017). These factors are in general used in a $1+1 \mathrm{D}$ approach, where the two sources of radiation are treated independently and scattering is neglected. Furthermore, they implicitly depend on the dust settling that was assumed during their derivation, as well as on the shape of the incident $u v$ field.

For better consistency, in this work we use a procedure that builds on our knowledge of the dust-attenuated $u v$ field. The extinction produced by the gas requires knowledge of the opacity

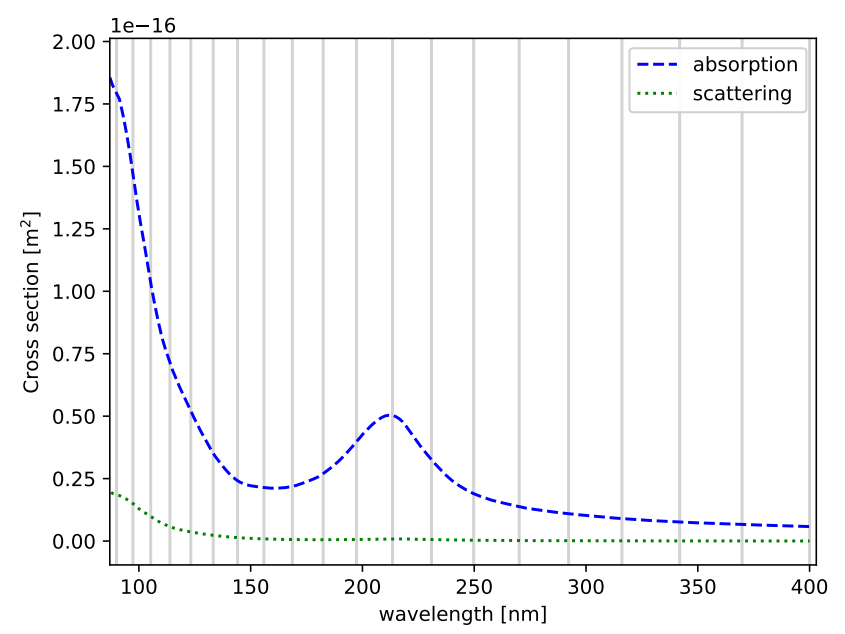

Fig. 6. Absorption (blue dashed line) and scattering cross sections (green dotted line) for the first grain size bin $(7 \mathrm{~nm})$. The vertical gray lines denote the wavelengths for which the radiation fields are simulated.

generated by the molecules through which the radiation travels from outside the disk to the local cell.

We show in Appendix B that a first order approximation for self and mutual shielding is obtained by applying the attenuation of the vertical opacity due to molecules to the radiation field computed with dust only by the POLARIS code, that is,

$I_{L}(\lambda, r, z)=\mathrm{e}^{-\tau_{\mathrm{m}}^{V}} I_{\mathrm{d}}(\lambda, r, z)$

where $I_{\mathrm{d}}(\lambda, r, z)$ is the radiation field given by POLARIS, which explicitly includes the impact of dust scattering, and $\tau_{\mathrm{m}}^{V}$ is the total opacity due to molecules from the $(r, z)$ point to $(r,+\infty)$.

\subsection{Chemistry simulations}

The NAUTILUS gas-grain code (Ruaud et al. 2016) is used to perform chemistry simulations. The NAUTILUS code is dedicated to computing chemical evolution in different interstellar environments and uses the rate equation approach (Hasegawa et al. 1992; Hasegawa \& Herbst 1993) to calculate the abundance as a function of time. The NAUTILUS gas-grain code is a three-phase model (Ruaud et al. 2016) that includes gas-phase chemistry and chemically active grain surfaces and mantles. Thus, aside from the gas-phase chemistry, NAUTILUS covers the physisorption of neutral species on the surface, diffusion of these species and their reactions, desorption of species from the surface, and repopulation of the surface by species from the mantle as the species on the surface evaporate. The latest version of NAUTILUS, the Nautilus Multi Grain Code (NMGC) OD model (Iqbal \& Wakelam 2018), can perform simulations using a full grain distribution in size. Each grain size bin is treated independently of the others and only interacts with gas. Compared to the single-grain model (model using a single-grain size), a multigrain model changes chemical rates in two ways. First, accretion rates of species depend on grain population. In a multi-grain model, accretion rates vary in different grain sizes according to their total surface area; Table A.1 shows the relative surface areas as function of grain sizes. Second, dust temperature depends on its size (see Fig. 3). Species have higher reaction rates on hotter grains as a result of higher hopping and desorption rates. In general, hotter grains have lower abundances of lighter species 
such as $\mathrm{CN}, \mathrm{CH}_{2}$, and $\mathrm{CO}$ and have higher abundances of heavier species such as $\mathrm{H}_{2} \mathrm{O}$ and $\mathrm{CH}_{3} \mathrm{OH}$ (see Iqbal \& Wakelam 2018, for details). In our current work, we generalize this multi-grain capability to a 1D situation to represent a protoplanetary disk as described in previous sections. NMGC computes chemistry in all cells given in the cylindrical coordinates $(r, z)$ for which we provide as input the gas temperature (Eq. (A.5)), local radiation field $I_{\mathrm{d}}(\lambda, r, z)$ and dust temperatures $T_{\mathrm{d}}\left(a_{i}, r, z\right)$, given by the radiative transfer simulations and number density of each dust population $n_{\mathrm{d}}\left(a_{i}, r, z\right)$ to compute the local grain abundances relative to the number density of hydrogen nuclei $n_{\mathrm{H}}(r, z)$ :

$A B_{\mathrm{d}}\left(a_{i}, r, z\right)=\frac{n_{\mathrm{d}}\left(a_{i}, r, z\right)}{n_{\mathrm{H}}(r, z)}$.

The $z$ direction is treated as in Hersant et al. (2009), but using 64 points.

\subsection{Photorates}

Radiations in the $u v$ range have a critical impact on the disk chemistry since $u v$ photons have the necessary energy to photoionize or photodissociate molecules in the disk. For this reason, characterizing the photorates $\left[\mathrm{s}^{-1}\right]$ at which molecules are dissociated or ionized is of high importance in chemistry models and necessary for a full interpretation of observations.

We evaluate the photo process rate as in Heays et al. (2017) as follows:

$k_{\mathrm{p}}(X, r, z)=\int_{\lambda} \sigma_{\mathrm{p}}(X, \lambda) I_{L}(\lambda, r, z) \mathrm{d} \lambda$,

where $\sigma_{\mathrm{p}}(X, \lambda)$ is the photo process cross section of species $X$ at wavelength $\lambda$ and $I_{L}(\lambda, r, z)$ is the local $u v$ radiation. The index $p$ equals $i$ when it stands for ionization and equal $d$ when it stands for dissociation. We perform our integration over the $91.2-400 \mathrm{~nm}$ wavelength range. To evaluate the rate at each point in the disk, we thus need the local $u v$ radiation field and the (space-independent) cross sections. Following Sect. 2.2.3, the $u v$ field is derived using Eq. (2).

The molecular cross sections used in this study are extracted from the Leiden University website ${ }^{1}$ (Heays et al. 2017). These cross sections are collected from experimental and theoretical studies. Eighty molecules are treated in this way. The photorates are recomputed at each explicit time step of the chemical evolution. For all other gas constituents (atoms or molecules unavailable in Heays et al. 2017), we use approximate shielding factors from Visser et al. (2009).

\subsection{Molecular hydrogen formation at the disk surface}

\subsubsection{The atomic $\mathrm{H}$ problem}

The NAUTILUS code initially implemented the $\mathrm{H}_{2}$ formation through the Langmuir-Hinshelwood (LH) mechanism that considers physisorption on grain surfaces. The LH mechanism is only efficient over a relatively narrow temperature range (5-15 K on flat surfaces Katz et al. 1999; Vidali et al. 2004, 2005).

In the case of multiple grain sizes, dust settling implies that only the small grains remain in the PDR regions of the disk. These small grains illuminated by the $u v$ field from the star can get significantly warmer at the disk surface, and the $\mathrm{H}_{2}$ formation

1 https://home.strw. leidenuniv.nl/ ewine/photo/cross_ sections.html via the $\mathrm{LH}$ mechanism as treated in most astrochemistry codes becomes inefficient. This leads to much smaller $\mathrm{H}_{2}$ formation rate than in the case of a single, larger, equivalent-area grain size with lower surface temperature. The low formation rate of $\mathrm{H}_{2}$ leaves a significant amount of atomic hydrogen that can severely affect the chemical balance in the disk upper layer (see Sect. 4 for a more detailed discussion).

Observations of $\mathrm{H}_{2}$ in unshielded regions (Habart et al. 2004, 2011), where dust can reach high temperatures $(\geq 20 \mathrm{~K})$, show that the column density can be significantly higher than what is expected by simple equilibrium rate equation and PDR model predictions. This implies that the formation mechanisms of $\mathrm{H}_{2}$ can be efficient in a wider domain than predicted by the $\mathrm{LH}$ mechanism at equilibrium temperature.

To evaluate the $\mathrm{H}_{2}$ formation rate, many studies adopt a canonical value for interstellar clouds derived by Jura (1975), which is defined by taking half the rate at which hydrogen atoms stick to the grain surface (e.g., Walsh et al. 2014; Agúndez et al. 2018).

Several other studies proposed more sophisticated mechanisms that theoretically produce comparable $\mathrm{H}_{2}$ column densities to those derived by observations (e.g., Duley 1996; Cazaux \& Tielens 2004; Cuppen \& Herbst 2005; Iqbal et al. 2012, 2014; Thi et al. 2020). We follow the approach of Bron et al. (2014), hereafter B14, which considers the stochastic fluctuations of the grain temperature and the $\mathrm{H}$-atom surface population.

\subsubsection{Effects of temperature fluctuations}

Small grains $(\leq 0.01 \mu \mathrm{m})$, given their very small heat capacity, have their temperature fluctuating widely even when a relatively small quantity of energy is absorbed. In typical unshielded regions, a small grain can absorb $u v$ photons originated from the interstellar field with a rather high probability, thereby causing a sudden spike in temperature. If the interval of time between $u v$ photon absorptions is sufficiently long, the grain that has undergone transient heating has the time to cool down to temperatures smaller than the equilibrium temperature, thereby giving the opportunity for $\mathrm{H}_{2}$ to form on the surface. Then, the rapid heating of the grain by the absorption of a $u v$ photon thermally desorbs most species on the surface, releasing $\mathrm{H}_{2}$ molecules to the gas phase.

Bron et al. (2014) studied the effect of these temperature fluctuations on the formation of $\mathrm{H}_{2}$ in PDRs and high $u v$ radiation regions using a statistical approach. By numerically solving the master equation (Le Bourlot et al. 2012), they show (Bron et al. 2014, see their Fig. 6) that the LH mechanism alone is sufficient to reach the typical $\mathrm{H}_{2}$ formation rates observed in PDRs when the temperature fluctuations of small grains are accounted for.

\subsubsection{Implementing the $\mathrm{H}_{2}$ formation rates}

The investigation of B14 lies in a domain of gas temperature $(100 \mathrm{~K})$ and gas density $\left(10^{0}\right.$ to $\left.10^{6} \mathrm{~cm}^{-3}\right)$ distinct from our study but this difference can easily be corrected because temperature and gas density only affect the results through the collision rate of atomic hydrogen with the grains, which scales as $n_{\mathrm{H}} \sqrt{T_{\mathrm{g}}}$. Thus, we can estimate the rate for a given density $n_{\mathrm{H}}$ and a given temperature $T_{\mathrm{g}}$ by extracting or interpolating from B 14 data the value corresponding to an equivalent gas density $n_{\text {eq }}$ equal to

$n_{\mathrm{eq}}=n_{\mathrm{H}} \sqrt{\frac{T_{\mathrm{g}}}{100 \mathrm{~K}}} \frac{s\left(T_{\mathrm{g}}\right)}{s(100 \mathrm{~K})}$, 


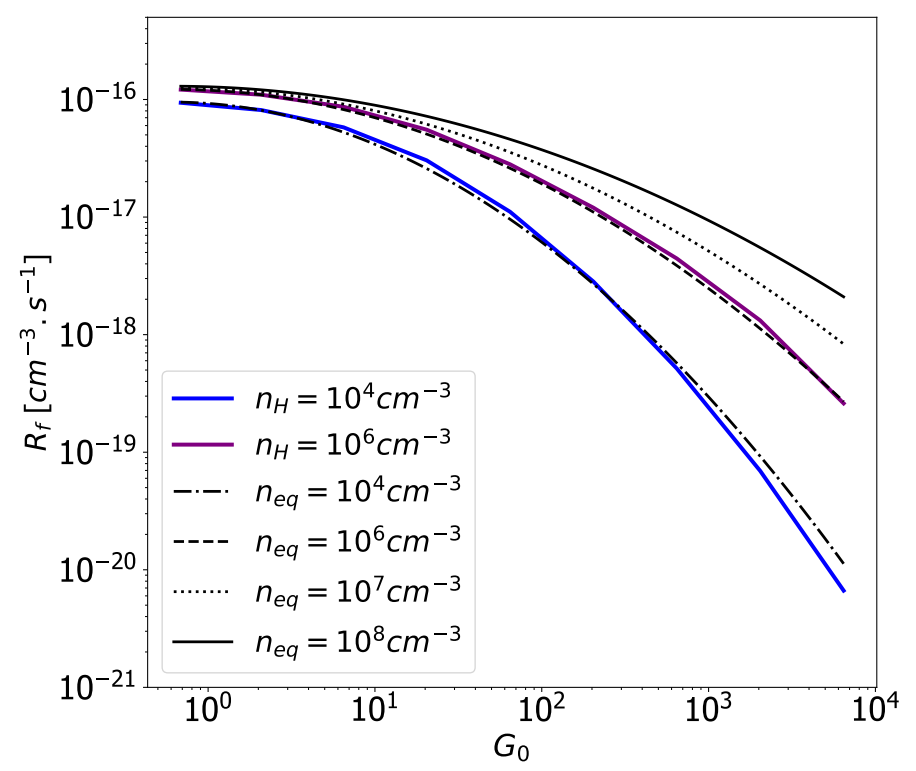

Fig. 7. $\mathrm{H}_{2}$ formation rate as a function of the $u v$ field intensity (in units of the Habing interstellar field) for various proton number density. Data adapted from Fig. 6 of Bron et al. (2014) are shown as the blue $\left(10^{4} \mathrm{~cm}^{-3}\right)$ and purple $\left(10^{6} \mathrm{~cm}^{-3}\right)$ solid lines, while black lines correspond to our analytic fit for four different number densities $\left(10^{4}, 10^{6}\right.$, $\left.10^{7}, 10^{8} \mathrm{~cm}^{-3}\right)$.

where $\mathrm{s}\left(T_{\mathrm{g}}\right)$ is the sticking coefficient at temperature $T_{\mathrm{g}}$ as defined by Le Bourlot et al. (2012),

$s(T)=\left(1+\left(\frac{T}{T_{2}}\right)^{\beta}\right)^{-1}$,

where $T_{2}=464 \mathrm{~K}$ and $\beta=1.5$, which approximately gives the same result as in Sternberg \& Dalgarno (1995).

We interpolated B14 data as a function of proton density and $u v$ field intensity using an analytic polylogarithmic function. The interpolation results are shown in Fig. 7. The PDR layers of protoplanetary disks are dense, typically above $10^{6}-10^{8} \mathrm{~cm}^{-3}$ so that essentially the upper curves in Fig. 7 apply to our case.

It should be noted that the B14 calculations assume a powerlaw size distribution from $1 \mathrm{~nm}$ to $300 \mathrm{~nm}$ composed of only carbonaceous grains. Changing the composition and/or size distribution would affect the precise values of the rates and fitted coefficients. However, the asymptotic behaviors of the rates should remain similar. The rate is proportional to the density at low densities and progressively saturates for larger densities as the fraction of atomic $\mathrm{H}$ decreases because the $u v$ flux is insufficient to dissociate $\mathrm{H}_{2}$. As a function of the $u v$ field intensity, the (unsaturated) rate is expected to decrease as $1 / G$ since the time spent by any grain in the relevant temperature range at which the LH formation is efficient is inversely proportional to the $u v$ photon flux.

\section{Model parameters and results}

\subsection{Detailed description of models}

We implement a set of 12 different models (see Table 3). All models share a common set of disk structure and physical parameters (see Fig. 2) as described in Table 1, but differ for a limited number of assumptions on grain sizes and temperatures, $u v$ field, or $\mathrm{H}_{2}$ formation mechanism.
The set is divided into three groups, that is, the single-grain models, multi-grain models, and an intermediate category of models with single grains locally varying to mimic the grain distribution used in the multi-grain model. We describe their specific characteristics in detail in Sects. 3.1.1, 3.1.2, and 3.1.3. As mentioned in Sect. 2.2.1, the excess of $u v$ radiation exhibited by typical T Tauri stars, given the strong wavelength-dependence of photo processes, inevitably impacts the chemistry of the disk. In order to have a broad view on how our chemical models react to different intensities of $u v$ flux, we adopt two regimes of radiation. One regime uses the spectrum of a relatively high- $u v$ emitting $\mathrm{T}$ Tauri star and a second regime uses the spectrum of a relatively low $u v$ emitting T Tauri star, hereafter called HUV and LUV, respectively. For the HUV regime we use the spectrum of TW Hya (Heays et al. 2017) while for the LUV we use that of DM Tau. Both far-ultraviolet (FUV) spectra are provided in the CTTS FUV spectra database ${ }^{2}$ (France et al. 2014). The blue solid line in Fig. B. 3 exhibits the spectrum of TW Hya as provided by France et al. (2014) before it is attenuated by the disk.

The chemical abundances in the disk are calculated using the time-dependent equation embedded in NAUTILUS. We use atomic initial abundances described in Table B.1 and all the chemical output are extracted after an evolution time of $5.10^{6} \mathrm{yr}$. We note that using molecular initial abundances would give almost the same final results for disks of this age (see Wakelam et al. 2019). All models incorporate the same chemical network and chemical processes described in Sect. 2.3. We ignore X-rays because of the large cumulative column densities found around the midplane, which is the main focus of this paper. X-rays would mostly affect the disk surface layers. We run all models using a standard cosmic-ray ionization rate of $1.3 \times 10^{-17} \mathrm{~s}^{-1}$ in the entire disk (see Cleeves et al. 2015, for a detailed discussion on the ionization rate).

\subsubsection{Single-grain models}

We use single-grain models as a comparison to our more sophisticated multi-grain models described in Sect. 3.1.2. These models are treated as in Wakelam et al. (2016), in particular with respect to the $u v$ field penetration and photo processes (see Hersant et al. 2009). We use the same gas density distribution and same overall gas-to-dust ratio (and hence the same dust mass) as in the multi-grain models. All single-grain models have a single dust size of $0.1 \mu \mathrm{m}$. The grains are settled toward the midplane assuming a dust scale height as defined in Eq. (A.20). However, $0.1 \mu \mathrm{m}$ grains exhibit little settling, as shown in Fig. A.1, and such models are virtually equivalent to non-settled dust models (Wakelam et al. 2016). Abundance maps of simple molecules are presented in Figs. 8 and D.2.

Model 1: $x U V-L H-T_{g}$. The dust temperature $\left(T_{\mathrm{d}}\right)$ profile follows that of the gas $\left(T_{\mathrm{g}}\right)$ (given by the solid black line in Fig. 5); $T_{\mathrm{d}}$ is equal to $T_{\mathrm{g}}$ everywhere in the disk. The quantity $x$ means either $\mathrm{H}$ for high or $\mathrm{L}$ for low, hence Model 1 is either HUVLH- $T_{\mathrm{g}}$ or LUV-LH- $T_{\mathrm{g}}$, depending on whether the stellar $u v$ field is high (HUV) or low (LUV). The differences are detailed in Sect. 3.1.

Model 2: $x U V-L H-T_{a}$. Model 2 is similar to Model 1, except for the dust temperature. In Model 2, the grain temperature is given by Eq. (1) and shown in Fig. 5 (red solid line). The temperature $T_{\mathrm{d}}$ is hence the area-weighted temperature from the grain size distribution of the multi-grain models and it is called $T_{\mathrm{a}}$. As

2 https://cos.colorado.edu/ kevinf/ctts_fuvfield.html 
Table 2. Figures.

\begin{tabular}{lc}
\hline \hline Models & Figures \\
\hline Single, HUV & $10,11,12,13,15,16$, C.1 \\
Single, LUV & D.1, D.2, D.3 \\
Multi, HUV & $9,10,11,12,14,15,17,18,20,19$, C.2 \\
Multi, LUV & D.1, D.4 \\
Set, HUV & $9,12,15$, C.3, C.4 \\
\hline
\end{tabular}

$T_{\mathrm{a}}$ is significantly higher than $T_{\mathrm{g}}$, we expect depletion to occur at lower elevations than in Model 1.

Model 3: $x U V-B 14-T_{a}$. In Model 3, the prescription of Bron et al. (2014) as described in Sect. 2.5 is used to form $\mathrm{H}_{2}$. The expected effect is a higher production of $\mathrm{H}_{2}$ in the PDRs region of the disk than in the other models.

\subsubsection{Multi-grain models}

Multi-grain models, as opposed to single-grain models, include the full grain distribution detailed in Table A.1, where each grain population has a specific temperature (Fig. 5), a specific settling factor, and is chemically active. For all multi-grain models, we use a size range of [5 $\mathrm{mm}, 1 \mathrm{~mm}$ ] with a size exponent $d=-3.5$ (Eq. (A.16)) and divide the grain size interval into 16 logarithmically distributed subintervals using Eq. (A.19). An overview of the grain size interval with their respective relative dust mass and surface area is given in Table A.1 while Fig. A.1 shows the scale height of each 16 grain size population as a function of the radius. The abundance maps of simple molecules are presented in Fig. 9. Multi-grain models are composed of two categories.

Model 4: M-xUV-LH. Either called M-HUV-LH or M-LUVLH depending on the flux, they use the classical LH mechanisms for $\mathrm{H}_{2}$ formation without considering small grains temperature fluctuation.

Model 5: M-xUV-B14. We add the $\mathrm{H}_{2}$ formation mechanisms prescribed in B14 (see Sect. 2.5). These models, with grain growth, dust settling, one temperature per grain size, and $\mathrm{H}_{2}$ formation derived from Bron et al. (2014), are the most complex models we compute.

\subsubsection{Intermediate models}

These models use locally a single-grain size mimicking the grain growth and dust settling of the multi-grain models (see Appendix C.1 for details). At each point, the single-grain size, which is chosen to obtain the same grain mass and area, are summed up over the grain size distribution of the multi-grain models. The purpose of these models is to better illustrate the impact of a dust temperature spread onto the chemistry; therefore we only run these models at radii 50, 100, and $200 \mathrm{au}$.

Model 6: Set-HUV-y-Ta. There are two models. The dust temperature is $T_{\mathrm{a}}$ while the $\mathrm{H}_{2}$ formation (y) either follows the LH mechanism or the B14 approximation.

Table 3 summarizes the various models. Table 2 lists the different figures in the text in relation with their respective models. A few figures relevant for the HUV cases are presented in Appendix C, while all figures for the LUV cases appear in Appendix D.

A65, page 8 of 39

\subsection{Results}

In this section, we present some general issues about the $u v$ field, dust temperature, and $\mathrm{H}_{2}$ formation. The next section has a more thorough discussion of the chemistry.

\subsubsection{Impact of $u v$ field}

A comparison between Tables model, the integrated column densities (or molecular surface densities) have about the same order of magnitude whether the flux is high (HUV) or low (LUV). Unlike in the PDR layer, the midplane chemistry is not dependent on the $u v$ flux regime. The dust opacity is such that the $u v$ flux is sufficiently attenuated in the HUV and LUV models for the chemistry to be similar in both cases.

Figures C.1, C.2, D.3, and D.4 show that the chemistry is nearly the same for all models from the midplane to roughly 1.5 $\mathrm{H}$. We observe more $\mathrm{H}_{2}, \mathrm{CO}, \mathrm{CS}$, and $\mathrm{CN}$ above three scale heights in the LUV models than in the HUV models because a high $u v$ flux implies more efficient photodissociation. Near the midplane, the $u v$ field is similarly attenuated and the radiation field intensities are about the same in all models. As a consequence, the only parameter that impacts the chemistry is the dust temperature $T_{\mathrm{d}}$ and this applies both to the single-grain models and the multi-grain models.

\subsubsection{Impact of dust temperature on $\mathrm{H}_{2}$ formation and chemistry}

A first effect of the adopted grain temperature is related to the formation of $\mathrm{H}_{2}$ and the remaining amount of $\mathrm{H}$, which is very important for the gas-phase chemistry. Adopting the formalism of Bron et al. (2014) to form $\mathrm{H}_{2}$ produces, as expected, more $\mathrm{H}_{2}$. In these cases, Figs. C. 1 and C. 2 show a shift in the $\mathrm{H} / \mathrm{H}_{2}$ transition toward the disk surface, which implies less gas-phase $\mathrm{H}$ and more $\mathrm{H}_{2}$ in the upper layers.

For single-grain models, the gas temperature we take is relatively low. Table 4 shows that when $T_{\mathrm{d}}=T_{\mathrm{g}}$, the remaining amount of $\mathrm{H}$ is still reasonably low (intermediate), contrary to the case in which $T_{\mathrm{d}}=T_{\mathrm{a}}$ (HUV-LH- $T_{\mathrm{a}}$ ), which exhibits the highest amount of $\mathrm{H}$, together with the multi-grain model $\mathrm{M}$ HUV-LH. Using only the $\mathrm{LH}$ mechanism to form $\mathrm{H}_{2}$ on grains with high temperatures significantly reduces the amount of $\mathrm{H}_{2}$ above two scale heights. This is particularly true for the M-HUVLH model, where the grain temperature depends on the grain size. The reservoir of "cold" grains is not large enough to allow for an efficient $\mathrm{H}_{2}$ formation on grains in the upper disk layer. On the contrary, near the midplane, in most models the grain temperature is always low enough for the LH mechanism to efficiently form $\mathrm{H}_{2}$ and regulate the atomic $\mathrm{H}$ abundance.

\section{Discussion}

We first discuss (Sect. 4.1) the vertical variations of abundances for $\mathrm{CO}, \mathrm{CN}$, and $\mathrm{CS}$. We then investigate the $\mathrm{C}, \mathrm{N}$, and $\mathrm{O}$ reservoirs and surface chemistry (Sects. 4.2-4.3) before discussing the formation of water and complex organic molecules (Sects. 4.4-4.5). We also discuss some possible implications of our results on planet formation and embryos compositions. We focus the discussion that follows (except for Sect. 4.1) on the HUV case (corresponding to the $u v$ flux received by the TW Hya disk) because we have seen that most of the analysis of HUV models can be considered valid for LUV models. Figures relevant to the LUV case (similar to what is expected for the DM Tau disk) are presented in Appendix D. All results are presented at the final stage of time evolution (i.e., $5 \times 10^{6} \mathrm{yr}$ ). 
S. Gavino et al.: Impact of size-dependent grain temperature on chemistry in protoplanetary disks
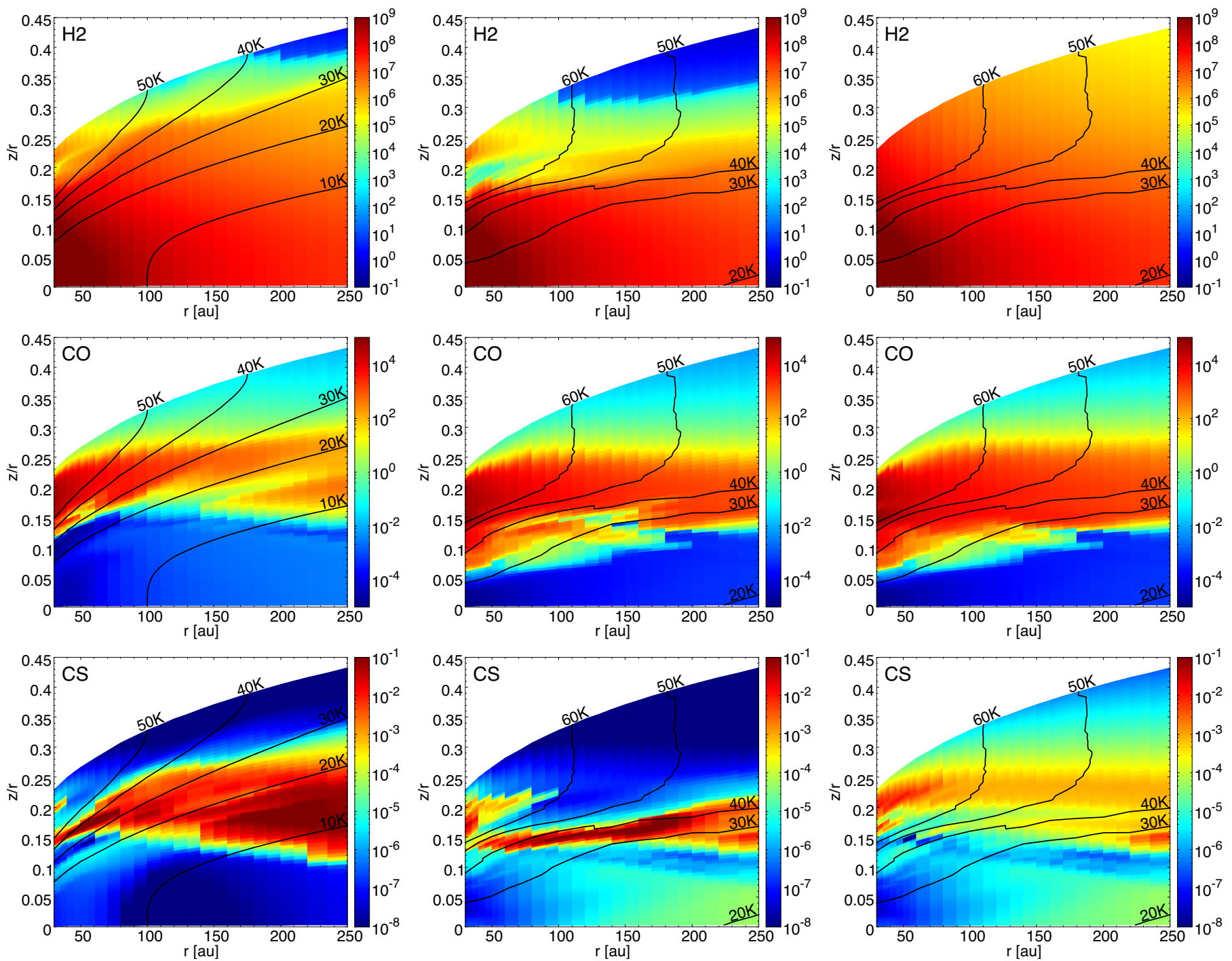

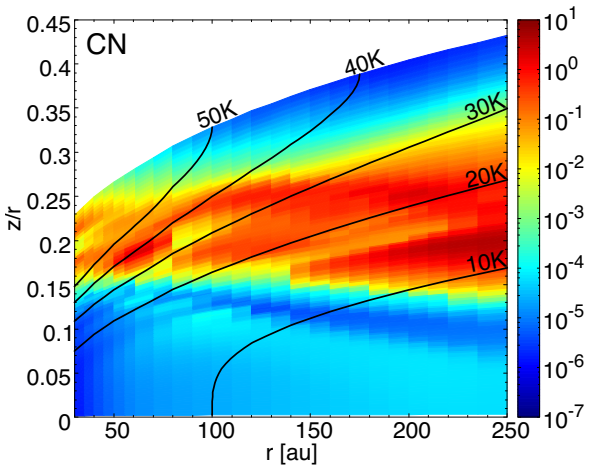

(a) HUV-LH-T

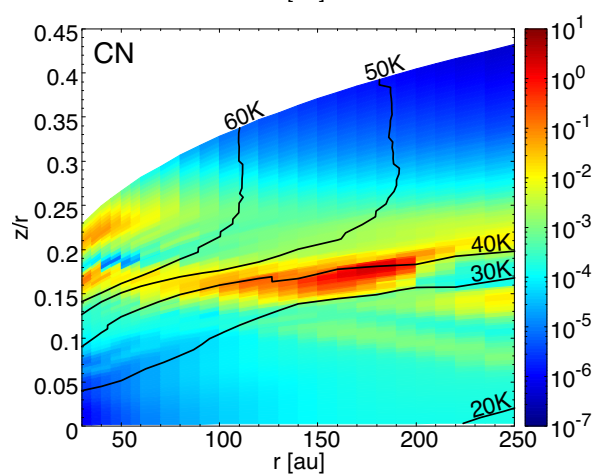

(b) HUV-LH-T

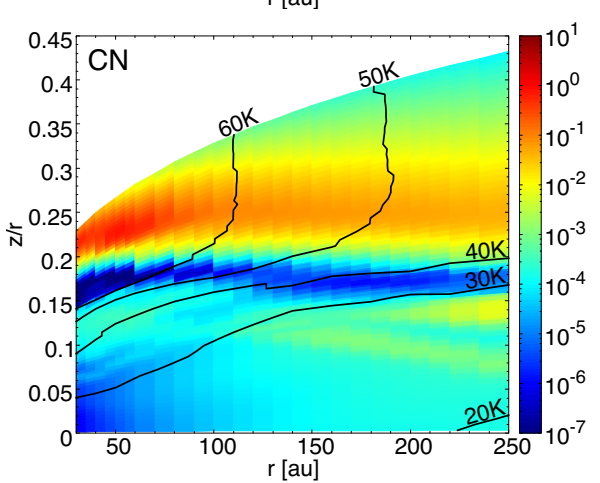

(c) HUV-B14-T

Fig. 8. Density $\left(\mathrm{cm}^{-3}\right)$ of $\mathrm{H}_{2}, \mathrm{CO}, \mathrm{CS}$ and $\mathrm{CN}$ in the gas phase of the single-grain models in HUV regime. Left column is the HUV-LH- $T_{\mathrm{g}}$ model, the middle column is HUV-LH- $T_{\mathrm{a}}$, and the right column is HUV-B14- $T_{\mathrm{a}}$. The black contours represent the dust temperature $\left(T_{\mathrm{d}}=T_{\mathrm{g}}\right.$ in the left column and $T_{\mathrm{d}}=T_{\mathrm{a}}$ in the middle and right columns).

\subsection{Vertical distribution of abundant (easily observed) molecules}

\subsubsection{Column densities}

Figure 10 shows the molecular surface densities of a few popular species detected in T Tauri disks. Tables 4 and D.1 present the column densities at a radius of 100 au for the most abundant species such as $\mathrm{CO}, \mathrm{CN}$, and $\mathrm{CS}$. These tables reveal that there is no specific trend for a given model, although there can be variations of up to 2.5 orders of magnitude in the column densities. An analysis of Figs. 11, C.1, and C.2, which provide the vertical profile of molecular densities at $100 \mathrm{au}$, is required to understand the origin of these variations.

For the column density of $\mathrm{CO}$ it shall be noted that $\mathrm{CO}$ is formed in the gas phase and is sensitive to photodissociation. The 

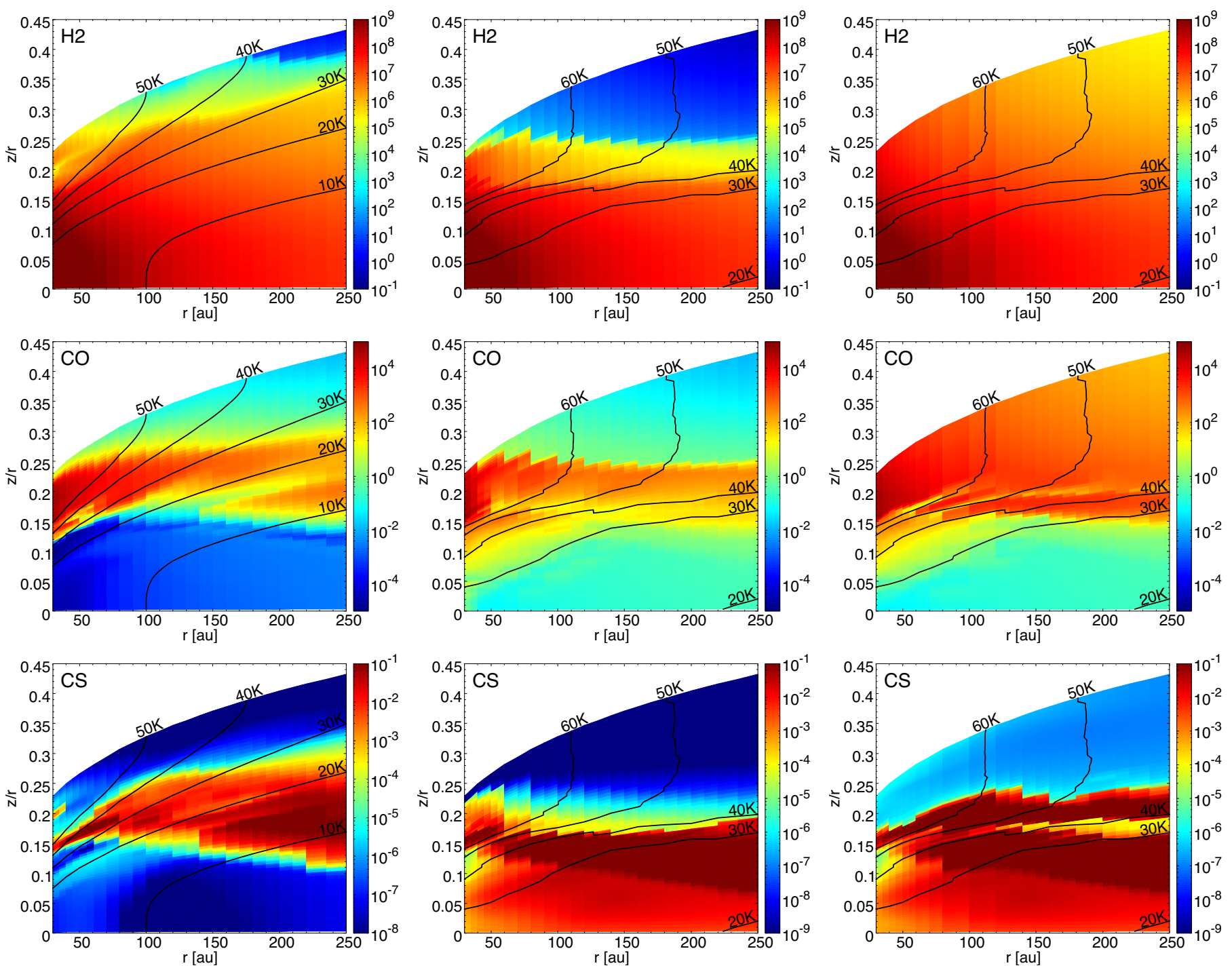

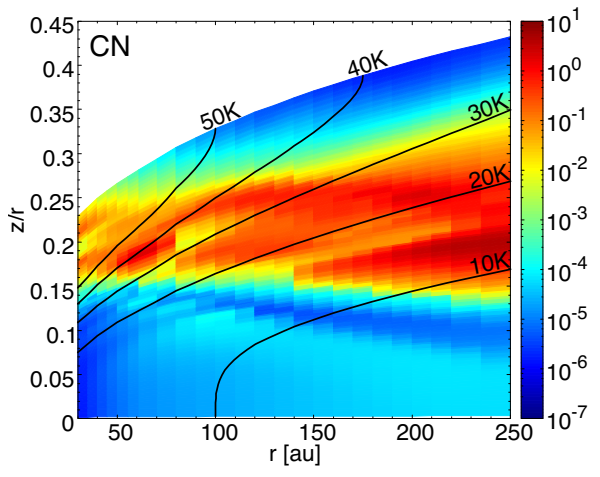

(a) HUV-LH-T

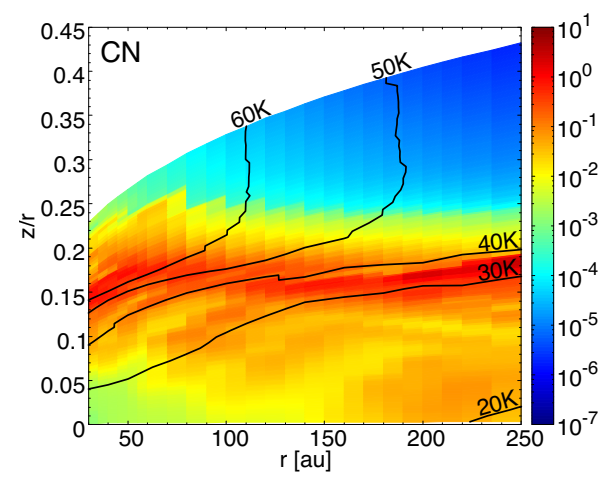

(b) M-HUV-LH

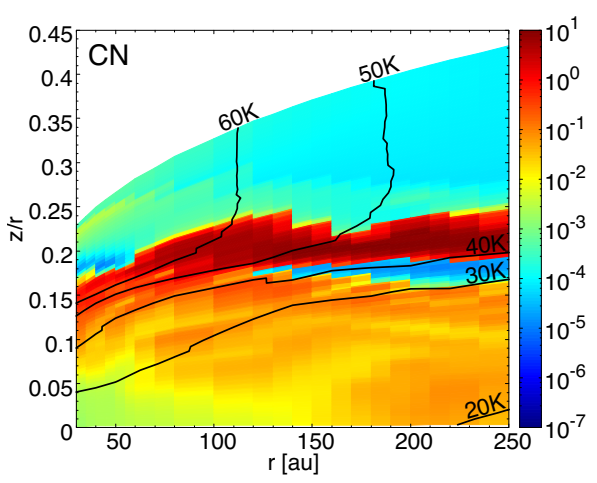

(c) M-HUV-B14

Fig. 9. Density $\left(\mathrm{cm}^{-3}\right)$ of $\mathrm{H}_{2}, \mathrm{CO}, \mathrm{CS}$, and $\mathrm{CN}$ in the gas phase of HUV-LH- $T_{\mathrm{g}}$ (left column) and of the multi-grain models in HUV regime. The black contours represent the dust temperature $\left(T_{\mathrm{d}}=T_{\mathrm{g}}\right.$ in the left column and $T_{\mathrm{d}}=T_{\mathrm{a}}$ in the middle and right columns).

model that produces the most CO is thus LUV-B14- $T_{\mathrm{a}}$ because this model combines both a low $u v$ flux, a large column density of $\mathrm{H}_{2}$ that shields $\mathrm{CO}$ from the $u v$, and a high grain temperature that prevents $\mathrm{CO}$ from being adsorbed. The second model producing the most $\mathrm{CO}$ is M-LUV-B14: the CO column density is slightly lower than in LUV-B $14-T_{\mathrm{a}}$ because in multi-grain models a fraction of the grains is significantly settled, involving a more effective $u v$ penetration. In that sense, it is not surprising that the M-HUV-LH model produces the smallest column density: this model combines a high $u v$ flux, more penetration from settling, and a small production of $\mathrm{H}_{2}$, which decreases the shielding.

For the column densities of $\mathrm{CS}$ and $\mathrm{CN}$, Table 4 shows that using predicted molecular surface densities is not enough to 
Table 3. Description of models.

\begin{tabular}{lcccccc}
\hline \hline & Bins & Sizes $(\mu \mathrm{m})$ & $T_{\mathrm{d}}$ & HUV & LUV & $\mathrm{H}_{2}$ formation \\
\hline HUV-LH- $T_{\mathrm{g}}$ & 1 & 0.1 & $T_{\mathrm{g}}$ & $\checkmark$ & & LH \\
HUV-LH- $T_{\mathrm{a}}$ & 1 & 0.1 & $T_{\mathrm{a}}$ & $\checkmark$ & & LH \\
HUV-B14- $T_{\mathrm{a}}$ & 1 & 0.1 & $T_{\mathrm{a}}$ & $\checkmark$ & & B14 \\
${\text { LUV-LH- } \mathrm{a}_{\mathrm{g}}}$ & 1 & 0.1 & $T_{\mathrm{g}}$ & & $\checkmark$ & LH \\
LUV-LH- $T_{\mathrm{a}}$ & 1 & 0.1 & $T_{\mathrm{a}}$ & & $\checkmark$ & LH \\
LUV-B14- $T_{\mathrm{a}}$ & 1 & 0.1 & $T_{\mathrm{a}}$ & & $\checkmark$ & B14 \\
M-HUV-LH & 16 & {$\left[5.10^{-3}-10^{3}\right]$} & $T_{i\{i=1 \ldots 16\}}$ & $\checkmark$ & & LH \\
M-HUV-B14 & 16 & {$\left[5.10^{-3}-10^{3}\right]$} & $T_{i\{i=1 \ldots 16\}}$ & $\checkmark$ & & B14 \\
M-LUV-LH & 16 & {$\left[5.10^{-3}-10^{3}\right]$} & $T_{i\{i=1 \ldots 16\}}$ & & $\checkmark$ & LH \\
M-LUV-B14 & 16 & {$\left[5.10^{-3}-10^{3}\right]$} & $T_{i\{i=1 \ldots 16\}}$ & & $\checkmark$ & B14 \\
Set-HUV-LH- $T_{\mathrm{a}}$ & 1 & See Appendix C & $T_{\mathrm{a}}$ & $\checkmark$ & & LH \\
Set-HUV-B14- $T_{\mathrm{a}}$ & 1 & See Appendix C & $T_{\mathrm{a}}$ & $\checkmark$ & & B14 \\
\hline
\end{tabular}

Notes. HUV stands for high $u v$ field and LUV for low $u v$ field. LH is for the classical treatment of the Langmuir-Hinshelwood mechanism and B14 when the prescription of Bron et al. (2014) is used instead. $T_{\mathrm{g}}$ means that the dust temperature equals to that of the gas, $T_{\mathrm{a}}$ is the weighted-area dust temperature as defined by Eq. (1). The parameter $T_{i}$ is the temperature of ith grain population, $\mathrm{M}$ stands for multi-grain models, and Set for dust models mimicking grain growth and dust settling similar to multi-grain models. As an example, the model M-HUV-B14 is a multi-grain model computed using the B14 method with a high $u v$ field.

Table 4. Column densities $\left(\mathrm{cm}^{-2}\right)$ of main molecules at 100 au for HUV models.

\begin{tabular}{|c|c|c|c|c|c|c|c|c|}
\hline & $\mathrm{H}$ & $\mathrm{H}_{2}$ & $\mathrm{CO}$ & $\mathrm{CS}$ & $\mathrm{CN}$ & $\mathrm{H}_{2}$ formation & $0-1.5 \mathrm{H}$ & $1.5-2.5 H$ \\
\hline HUV-LH- $T_{\mathrm{g}}$ & $1.48 \times 10^{21}$ & $8.19 \times 10^{22}$ & $3.24 \times 10^{17}$ & $4.40 \times 10^{12}$ & $3.79 \times 10^{13}$ & "cold" grains & $0 \%$ & $25 \%$ \\
\hline HUV-LH- $T_{\mathrm{a}}$ & $9.80 \times 10^{21}$ & $7.78 \times 10^{22}$ & $1.01 \times 10^{18}$ & $3.38 \times 10^{12}$ & $3.08 \times 10^{12}$ & More H & $100 \%$ & $100 \%$ \\
\hline HUV-B $14-T_{\mathrm{a}}$ & $3.20 \times 10^{20}$ & $8.25 \times 10^{22}$ & $1.46 \times 10^{18}$ & $1.35 \times 10^{11}$ & $1.34 \times 10^{13}$ & Less H & $100 \%$ & $100 \%$ \\
\hline M-HUV-LH & $8.26 \times 10^{21}$ & $7.86 \times 10^{22}$ & $7.46 \times 10^{16}$ & $6.69 \times 10^{13}$ & $4.62 \times 10^{13}$ & More $\mathrm{H}$ & $96 \%$ & $99 \%$ \\
\hline M-HUV-B14 & $1.31 \times 10^{20}$ & $8.26 \times 10^{22}$ & $9.02 \times 10^{17}$ & $1.92 \times 10^{14}$ & $3.49 \times 10^{14}$ & Less H & $96 \%$ & $99 \%$ \\
\hline Set-HUV-B14- $T_{\mathrm{a}}$ & $3.65 \times 10^{20}$ & $8.25 \times 10^{22}$ & $1.58 \times 10^{18}$ & $3.96 \times 10^{12}$ & $3.96 \times 10^{13}$ & Less $\mathrm{H}$ & $100 \%$ & $100 \%$ \\
\hline
\end{tabular}

Notes. The last three columns summarize the main properties of the model with respect to the $\mathrm{H}_{2}$ formation and grains (\% of grains, in sites, with a temperature above $20 \mathrm{~K}$, between 0 and $1.5 \mathrm{H}$, and between 1.5 and $2.5 \mathrm{H}$ ).

derive general trends. Nevertheless, we can note the M-HUV$\mathrm{B} 14$ model produces the most $\mathrm{CS}$ and $\mathrm{CN}$. On the other hand, HUV-B14- $T_{\text {a }}$ produces the smallest column density of CS while the HUV-LH- $T_{\mathrm{a}}$ model produces the smallest column density of $\mathrm{CN}$. This suggests routes of formation that do not simply depend on the grain temperature.

In the case of the single-grain models (see Figs. C.1 and D.3), all models using the same grain temperature $\left(T_{\mathrm{d}}=T_{\mathrm{g}}\right.$ or $\left.T_{\mathrm{a}}\right)$ have the same vertical profile at altitudes $z<1.5 H$ regardless of the flux regime. Similarly, since all multi-grain models use exactly the same grain temperature and size distribution, they all exhibit the same vertical density profile at altitudes $z<1.5 H$ (for a given species) regardless of the incident flux and of the assumed $\mathrm{H}_{2}$ formation mechanism (see for example, the $\mathrm{CO}$ abundance profiles for the two multi-grain models in Fig. C.2, middle panels of columns b-c). Accordingly, in this relatively high-mass disk, differences in column densities (Table 4) result from what happens above $z=1.5 \mathrm{H}$.

Finally, concerning the intermediate model, Set-HUV-B14$T_{\mathrm{a}}$, Table 4 shows that the column densities are rather similar to those found for model HUV-LH- $T_{\mathrm{a}}$, with the exception of CS where the column density is closer to that found for HUVLH- $T_{\mathrm{g}}$. However, these values are integrated column densities and Figs. C. 3 and C. 4 show that the vertical profiles are more complex than the comparison based on surface densities may suggest.

\subsubsection{Vertical variations}

CO. Gas-phase $\mathrm{CO}$ abundance is influenced by grain temperature and by abundance of $\mathrm{H}_{2}$. For multi-grain models, the flux penetration is larger than in single-grain models, so that CO column densities appear to be globally smaller than in single-grain models (Fig. 10). In M-HUV-LH, the CO abundance remains low above $z \sim 3 H$ (see Fig. C.2). On the other hand, M-HUV-B14 produces more $\mathrm{H}_{2}$ allowing $\mathrm{CO}$ to survive at higher altitudes. The resulting $\mathrm{CO}$ column density is of the same order as in HUV-LH- $T_{\mathrm{a}}$ and HUV-B14- $T_{\mathrm{a}}$. Although this has little impact on the total column density, the abundance of $\mathrm{CO}$ at $z \leq 1 \mathrm{H}$ is much higher in multi-grain models than in 

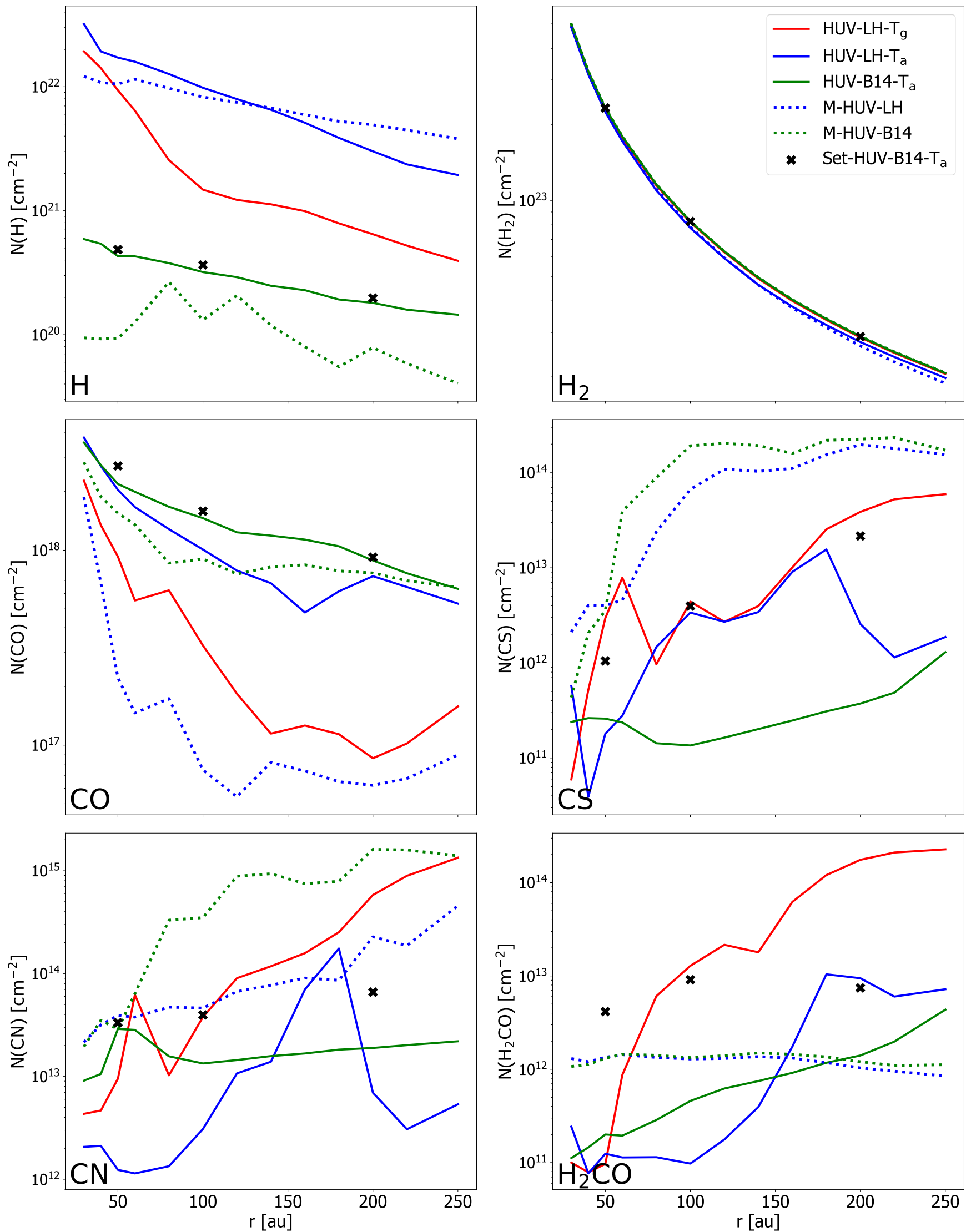

Fig. 10. Surface densities of the single-grain models (solid lines) and multi-grain models (dotted lines) as a function of the radius in high $u v$ flux regime. The crosses are the intermediate model for a selection of radii. 

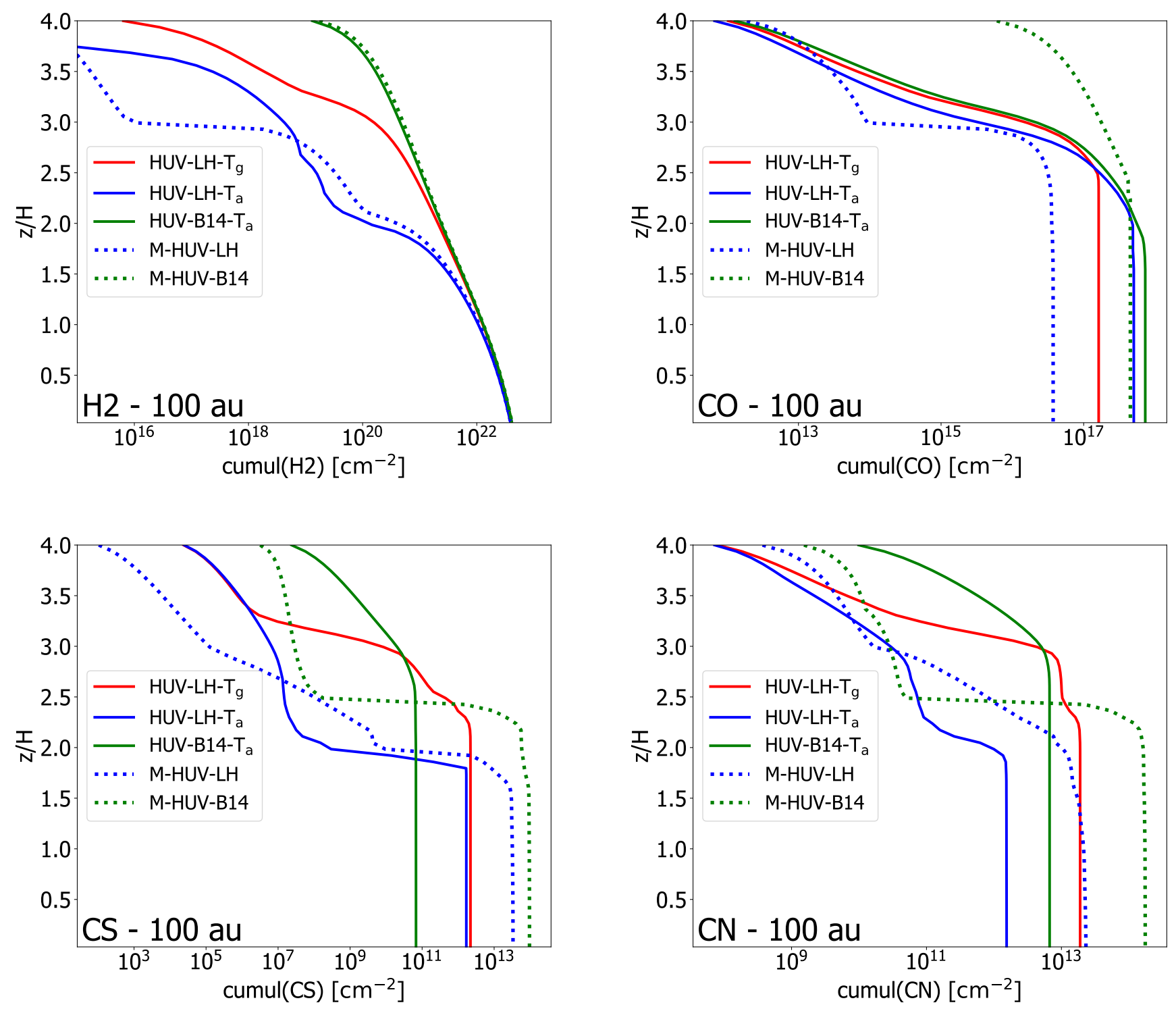

Fig. 11. Vertical cumulative surface density $\left(\mathrm{cm}^{-2}\right)$ of $\mathrm{H}_{2}, \mathrm{CO}, \mathrm{CS}$ and $\mathrm{CN}$ at 100 au from the star in the HUV models.

single-grain ones. In single-grain models, the single-grain temperature becomes low enough for $\mathrm{CO}$ to freeze out efficiently, while in multi-grain models there is always a fraction of abundant small grains whose temperature remains high enough to prevent $\mathrm{CO}$ from being depleted as efficiently as in single-grain models.

$C N$ and nitrogen bearing species. For all models, the main reactions that create $\mathrm{CN}$ in the upper layers at $100 \mathrm{au}$ are bimolecular reactions in the gas phase: $\mathrm{N}+\mathrm{C}_{2} \rightarrow \mathrm{C}+\mathrm{CN}$ $(69 \%)$ and $\mathrm{N}+\mathrm{CH} \rightarrow \mathrm{H}+\mathrm{CN}(22 \%)$. We note that $\mathrm{CH}$ is mostly destroyed by the reaction $\mathrm{H}+\mathrm{CH} \rightarrow \mathrm{C}+\mathrm{H}_{2}$. Therefore, more atomic $\mathrm{H}$ in the upper layers implies less $\mathrm{CH}$ available to form $\mathrm{CN}$. The effect is clearly visible for the single-grain models (Fig. C.1), where HUV-B14- $T_{\text {a }}$ produces more $\mathrm{CN}$ above $3.5 \mathrm{H}$ because $\mathrm{H}_{2}$ is more abundant compared to HUV-LH- $T_{\mathrm{a}}$. The effect is less obvious in the multi-grain models (C.2). Below $z=1.5 \mathrm{H}$, all the single-grain models exhibit roughly the same abundance, including HUV-LH- $T_{\mathrm{g}}$, which suggests a low sensitivity of $\mathrm{CN}$ due to the dust temperature in this range of values.
CS. The main formation process of CS in the upper layers is a sequence of a bimolecular reaction and a dissociative recombination reaction: $\mathrm{H}_{2}+\mathrm{CS}^{+} \rightarrow \mathrm{H}+\mathrm{HCS}^{+}$and $\mathrm{HCS}^{+}+\mathrm{e}^{-} \rightarrow \mathrm{H}+$ $\mathrm{CS}$. The sequence shows that the production of CS is dependent on $\mathrm{H}_{2}$. As a consequence, forcing the formation of $\mathrm{H}_{2}$ leads to the formation of $\mathrm{CS}$, providing that the ionization fraction is the same (as it is the case here).

For single-grain models, the grain temperature is higher in HUV-LH- $T_{\mathrm{a}}$ than in HUV-LH- $T_{\mathrm{g}}$. Because the production of $\mathrm{H}_{2}$ is more efficient in the latter model at high elevation, the peak of CS in HUV-LH- $T_{\mathrm{g}}$ (Fig. C.1) is broader $(\sim 2$ to $3 H$ ). In the meantime, CS also tends to undergo sticking processes on surfaces higher in the disk, competing with its production in the gas phase. This explains the dramatic drop of CS density below $2 H$. As regards HUV-LH- $T_{\mathrm{a}}$, the production of $\mathrm{H}_{2}$ starts to be efficient at a lower scale height $(\sim 2$ versus $3 H)$. Then, $C S$ abundance dramatically increases at $\sim 1.8 H$ (because of the steep temperature slope) before rapidly decreasing as temperature is low enough for CS to deplete on the grains, resulting in a very narrow peak. In HUV-B14- $T_{\mathrm{a}}$, the production of $\mathrm{H}_{2}$ is efficient even in the upper layers and CS starts to form higher in the disk, 
explaining why the CS abundance is two orders of magnitude larger than in HUV-LH- $T_{\mathrm{g}}$ and HUV-LH- $T_{\mathrm{a}}$ at $4 H$ (Fig. C.1).

For multi-grain models, we find a larger CS column density but the location of the peak of abundance is lower in altitude (Fig. 11c) because of the relatively low visual extinction. Just as for the single-grain models, the surface density is larger in the case of M-HUV-B14 because the formation of $\mathrm{H}_{2}$ is more efficient and the quantity of $\mathrm{H}$ lower. Below $z=1.5 \mathrm{H}$, the difference in abundance is strictly dependent on $T_{\mathrm{d}}$ and as expected, the hotgrain models (HUV-LH- $T_{\mathrm{a}}$ and HUV-B14- $T_{\mathrm{a}}$ ) produce more CS in the gas phase than the cold-grain model HUV-LH- $T_{\mathrm{g}}$, whereas all multi-grain models exhibit the same vertical profile.

Concerning the intermediate models (Set-HUV-B14- $T_{\mathrm{a}}$ and Set-HUV-LH- $T_{\mathrm{a}}$ ), Fig. C. 3 and Fig. C. 4 show that above the altitude of $z \sim 2 H$, these models are, as expected, very similar to what is observed for models HUV-LH- $T_{\mathrm{a}}$ because they share the same grain temperature for a number of grain sites, which is of the same order. Beyond $z \sim 2 H$, the local gas densities for $\mathrm{CO}, \mathrm{CN}$, and $\mathrm{CS}$ are closer to what is found for the multi-grain models. This is because in the intermediate models the number of grain sites around the midplane is lower than in the case of HUV-LH- $T_{\mathrm{a}}$ models and equal to those of multi-grain models. Moreover, the amount of $u v$ should be higher locally than in single-grain models because of dust settling. We note that in all cases, at the midplane, the abundance of gas species is always very low, well below observational levels.

\subsection{Element reservoirs: $C, N, O$ in the cold midplane}

Because the relative importance of reservoirs appears roughly constant along the distance from the star, we decided to study the reservoirs at $100 \mathrm{au}$. All species that were investigated represent at least $0.5 \%$ of the total quantity of carbon, nitrogen, or oxygen. As previously seen, the chemistry below $z=1.5 \mathrm{H}$ mostly depends on the grain temperature. Therefore, it turns out to be sufficient to discuss HUV-LH- $T_{\mathrm{g}}$, HUV-LH- $T_{\mathrm{a}}$, M-HUV-LH, and Set-HUV-LH- $T_{\text {a }}$ only.

Carbon bearing species. Figure 12 (top) shows that frozen $\mathrm{CO}_{2}$ is the main carrier of carbon, both in HUV-LH- $T_{\mathrm{a}}$ and M-HUV-LH (and Set-HUV-LH- $T_{\mathrm{a}}$ ) with $58.1 \%$ and $51.3 \%$ of elemental carbon, respectively, while the quantity of $\mathrm{CO}_{2}$ is negligible in comparison to the other species in HUV-LH- $T_{\mathrm{g}}$. The dust temperature appears too high in these two models to allow for a large quantity of hydrogen to successively hydrogenate the frozen atomic oxygen to form water. Instead, the atomic oxygen easily diffuses on the surface and the relatively high temperature allows us to overcome the activation barrier to rapidly form $\mathrm{CO}_{2}$ by reacting with the frozen CO. In HUV-LH$T_{\mathrm{g}}$, on the other hand, Fig. 12 (top) shows that the main carrier of carbon is $\mathrm{CH}_{4}$ with $49.1 \%$. In this colder dust temperature model, $\mathrm{CO}$ is initially formed in the gas before being efficiently and rapidly adsorbed onto the grain surfaces, just like in hotgrain models. However, once on the surface, $\mathrm{CO}$ is channeled to $\mathrm{s}-\mathrm{CH}_{3} \mathrm{OH}$ through hydrogenation sequences. The photodissociation of $\mathrm{CH}_{3} \mathrm{OH}$ then leads to $\mathrm{CH}_{4}$ formation. Hence, in the midplane, disks with colder grains produce more $\mathrm{CH}_{4}$ while warmer disks produce more $\mathrm{CO}_{2}$. The other main carrier is the complex organic species $\mathrm{CH}_{3} \mathrm{OH}$, which holds about $10 \%$ of carbon in M-HUV-LH and HUV-LH- $T_{\mathrm{g}}$. Finally, it is important to mention that $\mathrm{CO}$ contributes as a reservoir of $\mathrm{C}$ for less than $0.5 \%$ in all models.

Oxygen-bearing species. $\mathrm{CO}_{2}$ ice is the main carrier of oxygen in HUV-LH- $T_{\mathrm{a}}$ (and Set-HUV-LH- $T_{\mathrm{a}}$ ) and M-HUV-LH
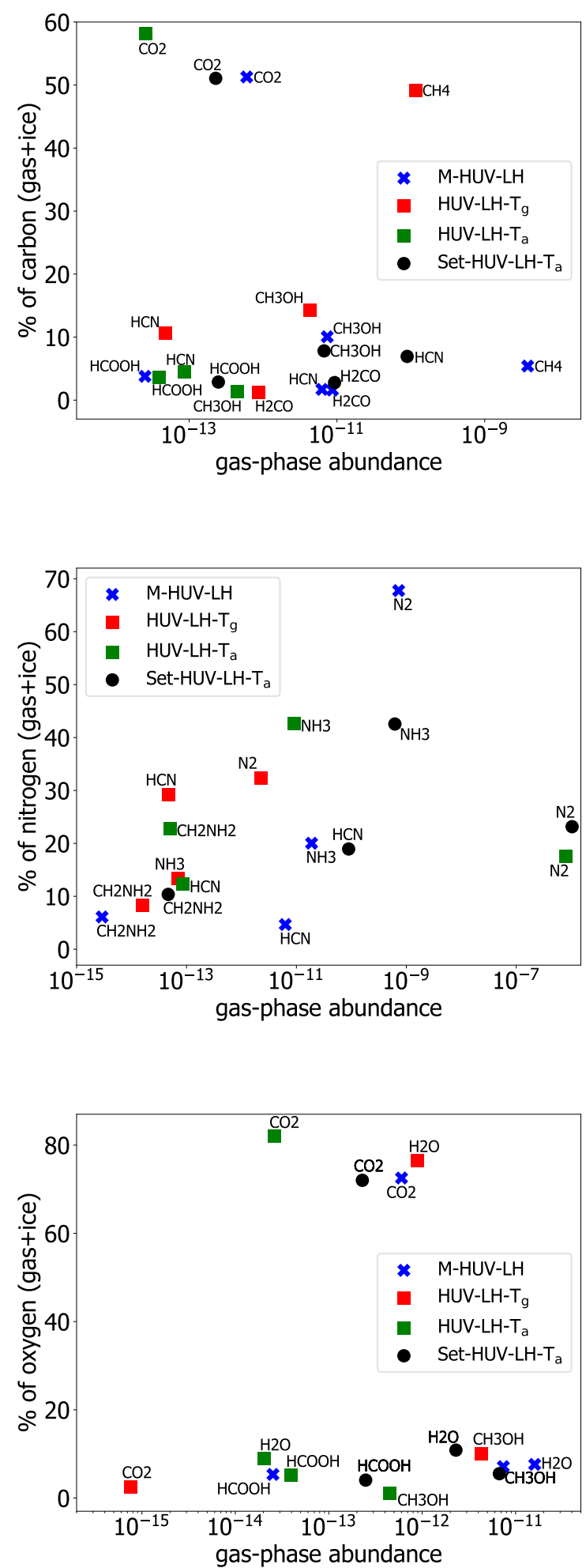

Fig. 12. Species that contain at least $0.5 \%$ of elemental carbon (top), nitrogen (middle), and oxygen (bottom) in the midplane at $100 \mathrm{au}$ : percentage of the carbon/nitrogen/oxygen locked in the species as a function of the gas-phase species abundance.

with $82.0 \%$ and $72.5 \%$, respectively (Fig. 12, bottom). The temperature of the grains in these models is too high $\left(T_{\mathrm{a}}=24.6 \mathrm{~K}\right)$ to allow for a large quantity of atomic hydrogen to remain on the grains and hydrogenate the atomic oxygen to form water. As described above, atomic oxygen easily diffuses and also combines with $\mathrm{CO}$ to form $\mathrm{CO}_{2}$. In HUV-LH- $T_{\mathrm{g}}, \mathrm{H}_{2} \mathrm{O}$ is by 
far the largest carrier of oxygen with $76.4 \%$. Disks with colder grains produce more $\mathrm{H}_{2} \mathrm{O}$ while disks with warmer grains produce more $\mathrm{CO}_{2}$. $\mathrm{CH}_{3} \mathrm{OH}$ is, again, an important carrier and holds about $10 \%$ of oxygen in HUV-LH- $T_{\mathrm{g}}$. As for C, CO holds less than $0.5 \%$ of oxygen in all models.

Nitrogen bearing species. Nitrogen is mostly in the form of $\mathrm{N}_{2}$ in M-HUV-LH (67.8\%) and in HUV-LH-T $T_{\mathrm{g}}(32.4 \%)$ (Fig. 12, middle). Moreover, in almost all models, the gas-phase abundance of $\mathrm{N}_{2}$ is noticeably larger than that of the other species. The reason for that is a combined effect of the faster conversion of atomic nitrogen into $\mathrm{N}_{2}$ in the gas phase compared to the depletion of $\mathrm{N}$ and the high grain temperature that prevents $\mathrm{N}_{2}$ from depleting onto the surfaces. The binding energy of $\mathrm{N}_{2}$ $\left(E_{\mathrm{b}}\left(\mathrm{N}_{2}\right)=1100 \mathrm{~K}\right)$ on amorphous water ice surface is smaller than most of the other molecules and slightly smaller than that of CO (Minissale et al. 2016; Wakelam et al. 2017), therefore $\mathrm{N}_{2}$ is retained more efficiently in the gas. We note that, given the low dust temperature, the gas-phase abundance of $\mathrm{N}_{2}$ in HUV-LH- $T_{\mathrm{g}}$ is the smallest of all models. In HUV-LH- $T_{\mathrm{a}}$ and in Set-HUV$\mathrm{LH}-T_{\mathrm{a}}$, more $\mathrm{N}_{2}$ remains in the gas phase because of the large dust temperatures. On the grains, $\mathrm{s}-\mathrm{NH}_{3}$ is the main carrier of nitrogen with $42.7 \%$ of the elemental nitrogen. Therefore, $\mathrm{N}_{2}$ is expected to be the carrier of nitrogen in the disk midplane but is more present in the gas phase in hot-grain models. The other main carriers of nitrogen are HCN $(29.2 \%$ in HUV-LH$T_{\mathrm{g}}, 12.3 \%$ in HUV-LH- $T_{\mathrm{a}}$ ) and $\mathrm{CH}_{2} \mathrm{NH}_{2}\left(22.9 \%\right.$ in HUV-LH- $T_{\mathrm{a}}$, $8.3 \%$ in HUV-LH- $T_{\mathrm{g}}$ ).

\subsection{Surface chemistry in the midplane}

One important conclusion of Sect. 4.2 is that disks with colder grains produce more $\mathrm{s}-\mathrm{CH}_{4}$ and $\mathrm{s}-\mathrm{H}_{2} \mathrm{O}$ in the midplane while disks with warmer grains produce more $\mathrm{s}-\mathrm{CO}_{2}$. This clearly demonstrates the major role of the grain temperature on the surface chemistry in the plane. We investigate more deeply the midplane surface chemistry of a few key molecules for the same four models (i.e., HUV-LH- $T_{\mathrm{g}}$, HUV-LH- $T_{\mathrm{a}}, \mathrm{M}-\mathrm{HUV}-\mathrm{LH}$, and Set-HUV-LH- $T_{\mathrm{a}}$ ). Another important conclusion is that our intermediate models, Set-HUV-LH- $T_{\mathrm{a}}$ and Set-HUV-B 14- $T_{\mathrm{a}}$, are not able to reproduce the complex behavior of the gas-grain chemistry observed with the multi-grain model, since it happens to be closer to the single-grain model HUV-LH- $T_{\mathrm{a}}$ in terms of main reservoirs in the midplane. Small differences occur for relatively minor constituents (s- $\mathrm{CO}, \mathrm{s}-\mathrm{H}_{2} \mathrm{CO}, \mathrm{s}-\mathrm{CH}_{3} \mathrm{OH}, \mathrm{s}-\mathrm{HCN}$, for example) because of the different effective dust area in the two cases (single vs. set), and hence different timescales for conversion on grain surfaces.

Figures 13 and 14 present maps of number density for s$\mathrm{H}_{2}$, s-CO, s-CS, and s-CN locked on grain surfaces in the case of single-grain and multi-grain models, respectively. Figure 15 presents the surface abundance per total atomic hydrogen for various molecules as a function of the grain radius $a$ and their temperature in the midplane of the disk. The crosses, square markers, and round markers stand for the multi-grain model at $30 \mathrm{au}, 100 \mathrm{au}$, and $200 \mathrm{au}$ respectively. The triangle pointing upward represents the surface abundance in the single-grain model HUV-LH- $T_{\mathrm{g}}$ and the triangle pointing downward represents the abundance in the single-grain model HUV-LH- $T_{\mathrm{a}}$, both at $100 \mathrm{au}$.

\subsubsection{Carbon and oxygen-bearing molecules: $\mathrm{CO}, \mathrm{CO}_{2}$ and $\mathrm{CH}_{4}$}

$\mathrm{CO}$ is formed in the gas phase prior to being accreted on small grains during the first $10^{6} \mathrm{yr}$ of the simulation. However, because of its small binding energy $\left(E_{\mathrm{b}}(\mathrm{CO})=1300 \mathrm{~K}\right.$; Wakelam et al. 2017), CO is rapidly thermally desorbed from the hot $\left(T_{\mathrm{g}}>\right.$ $20 \mathrm{~K}$ ) smaller grains and gets accreted by large cold grains $\left(T_{\mathrm{g}}<20 \mathrm{~K}\right)$, where it remains locked until the simulation is stopped. Considering this mechanism, $\mathrm{CO}$ is distributed according to the grain population and Fig. 15 (top left panel) shows two regimes of abundance, accounting for the desorption barrier, where $\mathrm{CO}$ ice tends to stay locked on grains of size $\gtrsim 1 \mu \mathrm{m}$ at all radii.

For the single-grain models, the same mechanism is at play. However, rather than involving a distribution of s-CO on various grain populations, this simply results in a small abundance in the hot-grain model HUV-LH- $T_{\mathrm{a}}$ and a high abundance in the cold-grain model HUV-LH- $T_{\mathrm{g}}$.

As for $\mathrm{CO}_{2}$, its binding energy is relatively high $\left(E_{\mathrm{b}}\left(\mathrm{CO}_{2}\right)=2600 \mathrm{~K}\right.$; Wakelam et al. 2017). As a consequence the abundance of s- $\mathrm{CO}_{2}$ depends very little on the dust temperature and simply depends on the dust surface area instead. $\mathrm{s}-\mathrm{CO}_{2}$ is efficiently formed through

$$
\begin{aligned}
& \mathrm{s}-\mathrm{O}+\mathrm{s}-\mathrm{CO} \rightarrow \mathrm{s}-\mathrm{CO}_{2}\left(\mathrm{E}_{\mathrm{A}}=1000 \mathrm{~K}\right) \\
& \mathrm{s}-\mathrm{OH}+\mathrm{s}-\mathrm{CO} \rightarrow \mathrm{s}-\mathrm{CO}_{2}+\mathrm{s}-\mathrm{H} .
\end{aligned}
$$

Therefore, $\mathrm{s}-\mathrm{CO}_{2}$ is more abundant in the smaller grains because their temperature allows these grains to overcome the activation barrier of reaction 7 and the drop in abundance in large grains at $200 \mathrm{au}$ (and further) originates from the grain temperature getting too low for reaction 7 to be activated. Therefore, the outer disk forms less $\mathrm{CO}_{2}$ ice.

In cold-grain models, $\mathrm{s}-\mathrm{CH}_{4}$ is the largest carrier of carbon (see Sect. 4.2). As expected, Fig. 15 (Bottom-left) shows that $\mathrm{s}-\mathrm{CH}_{4}$ is more abundant in cold grains $\left(T_{\mathrm{g}}<20 \mathrm{~K}\right) . \mathrm{s}-\mathrm{CH}_{4}$ is formed via the hydrogenation of $\mathrm{s}-\mathrm{CH}_{3}$. The latter is formed through the following two sequences:

$$
\begin{aligned}
& \mathrm{s}-\mathrm{CO}+\mathrm{h} v \rightarrow \mathrm{s}-\mathrm{C}+\mathrm{s}-\mathrm{O} \\
& \mathrm{s}-\mathrm{H}_{2}+\mathrm{s}-\mathrm{C} \rightarrow \mathrm{s}-\mathrm{CH}_{2} \\
& \mathrm{~s}-\mathrm{H}_{2}+\mathrm{s}-\mathrm{CH}_{2} \rightarrow \mathrm{s}-\mathrm{CH}_{3}+\mathrm{s}-\mathrm{H}
\end{aligned}
$$

and

$$
\mathrm{s}-\mathrm{CH}_{3} \mathrm{OH}+\mathrm{h} v \rightarrow \mathrm{s}-\mathrm{OH}+\mathrm{s}-\mathrm{CH}_{3} .
$$

In view of the above, $\mathrm{s}-\mathrm{CH}_{3} \mathrm{OH}$ is formed on the grains prior to being photodissociated (if the computation domain is sufficiently extended). Then the released atomic carbon (Reaction 9) and s$\mathrm{CH}_{3}$ (Reaction 12) lead to $\mathrm{s}-\mathrm{CH}_{4}$ formation. Consequently, the formation of $\mathrm{s}-\mathrm{CH}_{4}$ is more efficient on large cold grains since (1) $\mathrm{CO}$ must be adsorbed and (2) hydrogenation is needed to create s- $\mathrm{CH}_{3} \mathrm{OH}$. s- $\mathrm{CH}_{4}$ ice is thus found to be more abundant on large grains and in the outer disk.

\subsubsection{Sulfur-bearing species: $\mathrm{H}_{2} \mathrm{~S}$}

In the gas phase, $\mathrm{H}_{2} \mathrm{~S}$ has been identified in dense cloud cores, cometary comae and Phuong et al. (2018) reported the first detection of $\mathrm{H}_{2} \mathrm{~S}$ in the cold and dense ring about the TTauri star GG Tau A. The low $\mathrm{H}_{2} \mathrm{~S}$ column densities observed in GG Tau A and in dense molecular clouds are assumed to be the result of a strong sulfur depletion (Hudson \& Gerakines 2018; Phuong et al. 2018).

We investigate here the formation routes of $\mathrm{S}-\mathrm{H}$ bonds and more specifically the simple S-bearing molecule hydrogen sulfide, $\mathrm{H}_{2} \mathrm{~S}$, in the midplane icy mantles of our multi-grain 

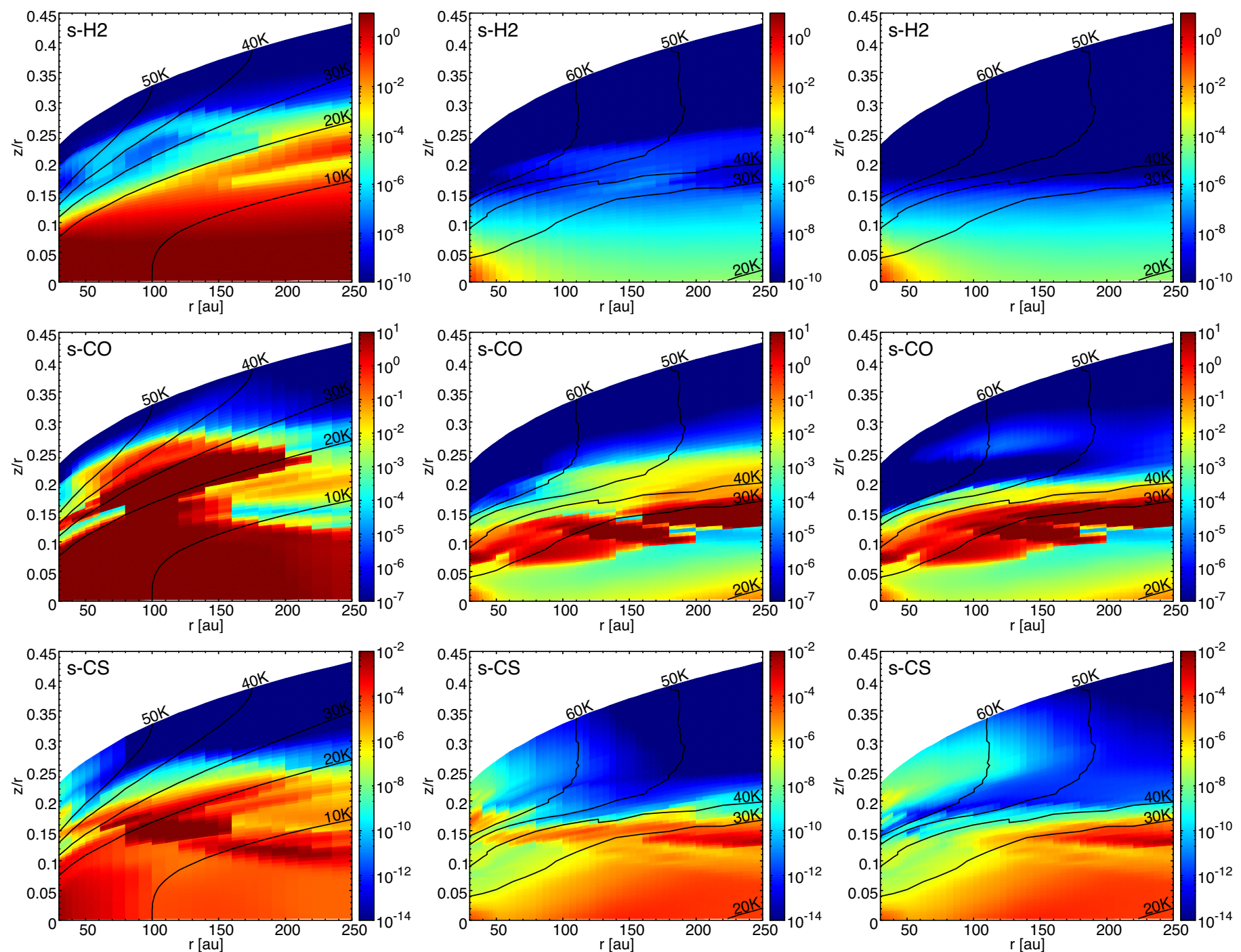

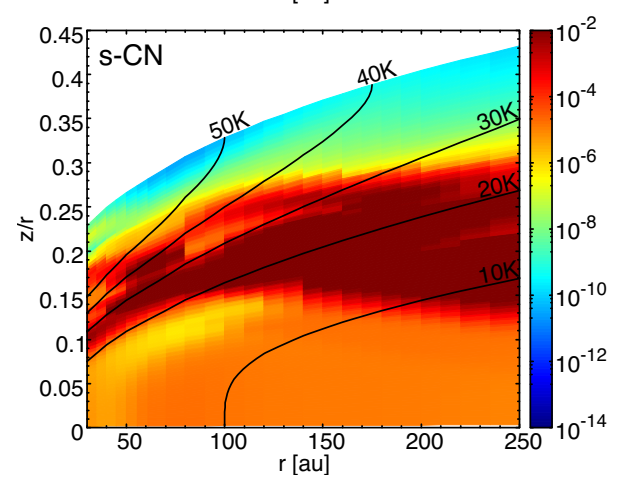

(a) HUV-LH-T

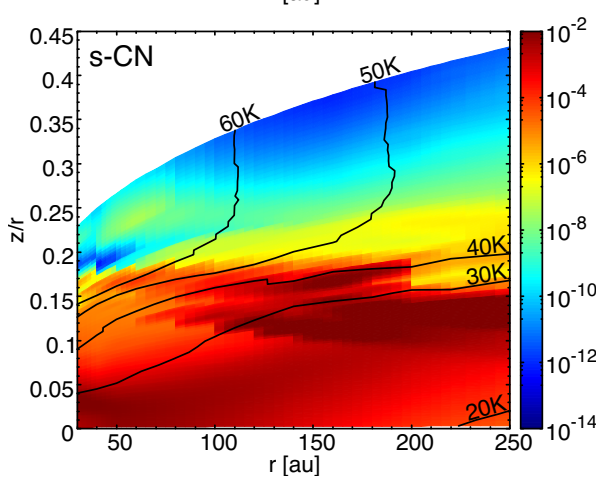

(b) HUV-LH-T

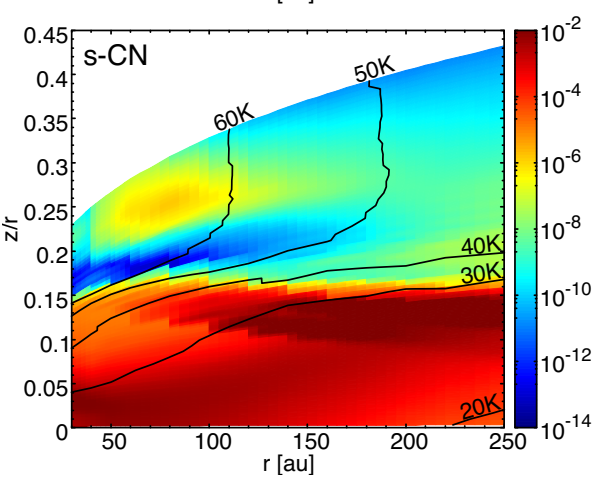

(c) HUV-B14-T

Fig. 13. Density $\left(\mathrm{cm}^{-3}\right)$ of $\mathrm{H}_{2}, \mathrm{CO}, \mathrm{CS}$, and $\mathrm{CN}$ in the gas phase of the single-grain models in the HUV regime. The left column shows the HUVLH- $T_{\mathrm{g}}$ model, middle column shows HUV-LH- $T_{\mathrm{a}}$ and right shows HUV-B14- $T_{\mathrm{a}}$. The black contours represent the dust temperature $\left(T_{\mathrm{d}}=T_{\mathrm{g}}\right.$ in the left column and $T_{\mathrm{d}}=T_{\mathrm{a}}$ in the middle and right columns).

models. Figure 15 (second row, right column) shows the abundance of $\mathrm{s}-\mathrm{H}_{2} \mathrm{~S}$ as a function of the grain size. There is a strong anti-correlation with grain temperatures. The smallest s$\mathrm{H}_{2} \mathrm{~S}$ abundances are located on the hottest grains $\left(T_{\mathrm{d}} \sim 27 \mathrm{~K}\right)$ while the largest abundances are found on large colder grains $\left(T_{\mathrm{d}} \lesssim 15 \mathrm{~K}\right)$. This anti-correlation is observed at all radii. The abundances on the large grains $(>10 \mu \mathrm{m})$ are independent of the radius. For the small grains, however, the inner disk exhibits larger abundances than the outer disk because of higher collision rates in the inner regions. The strong anti-correlation with temperature is directly related to the main formation pathways of $\mathrm{H}_{2} \mathrm{~S}$ that implies hydrogenation sequences:

$$
\begin{aligned}
& \mathrm{s}-\mathrm{S} \stackrel{\mathrm{s}-\mathrm{H}}{\longrightarrow} \mathrm{s}-\mathrm{HS} \stackrel{\mathrm{s}-\mathrm{H}}{\longrightarrow} \mathrm{s}-\mathrm{H}_{2} \mathrm{~S} \\
& \mathrm{~s}-\mathrm{CH}_{3} \mathrm{SH} \stackrel{\mathrm{s}-\mathrm{H}}{\longrightarrow} \mathrm{s}-\mathrm{CH}_{3}+\mathrm{s}-\mathrm{H}_{2} \mathrm{~S} .
\end{aligned}
$$


S. Gavino et al.: Impact of size-dependent grain temperature on chemistry in protoplanetary disks
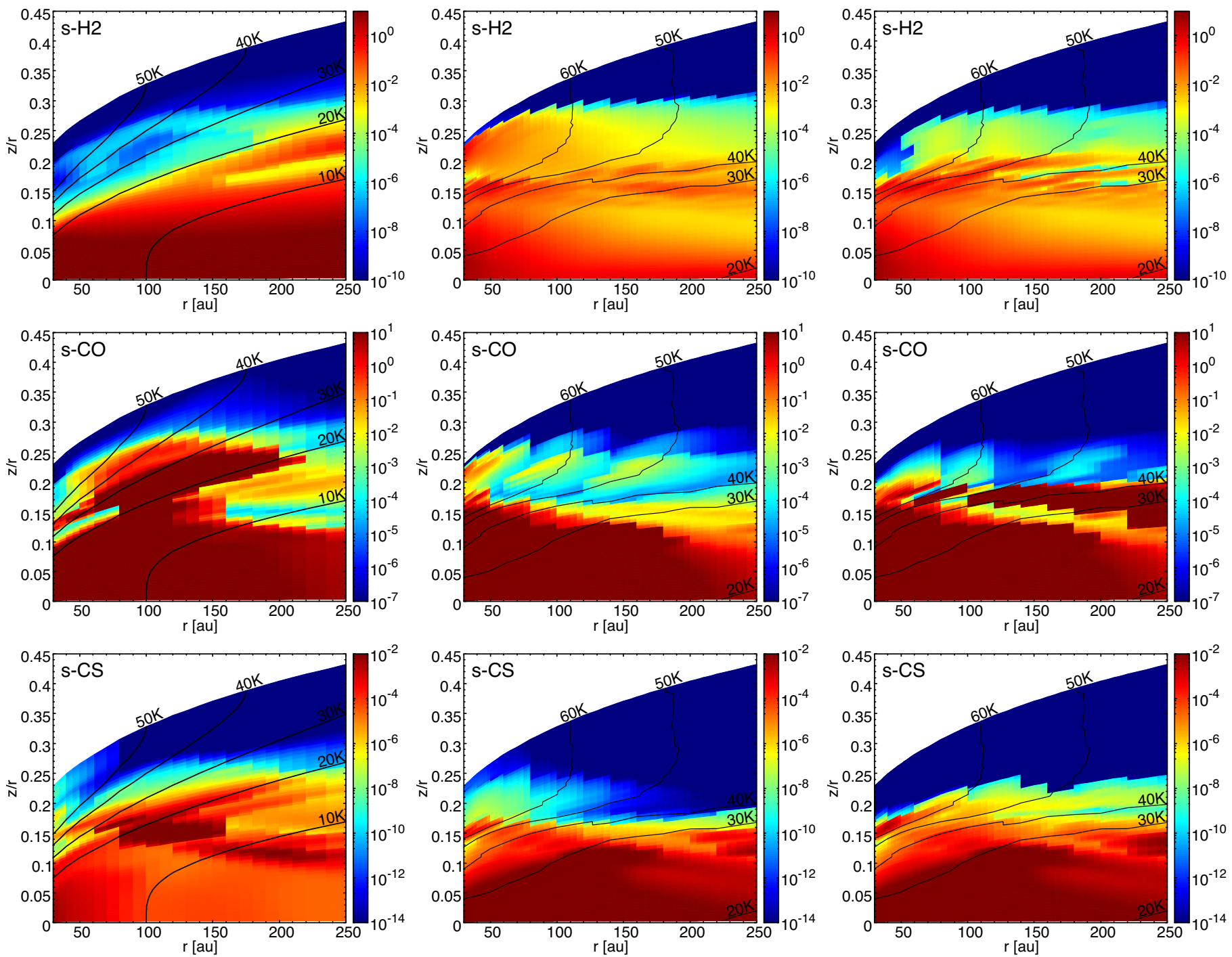

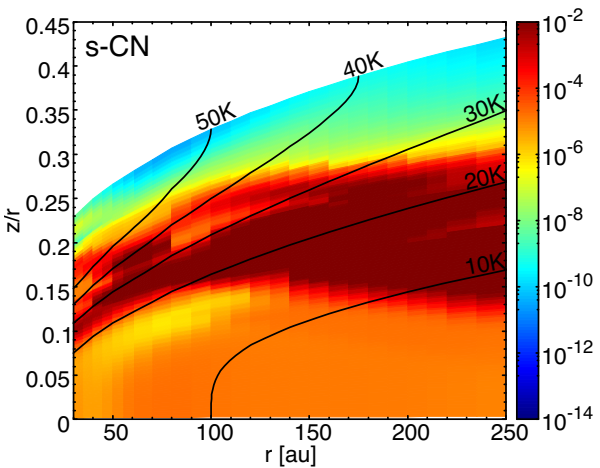

(a) HUV-LH-T $g$

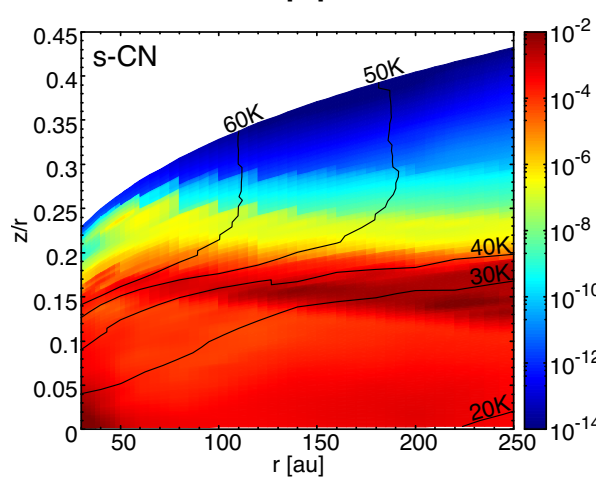

(b) M-HUV-LH

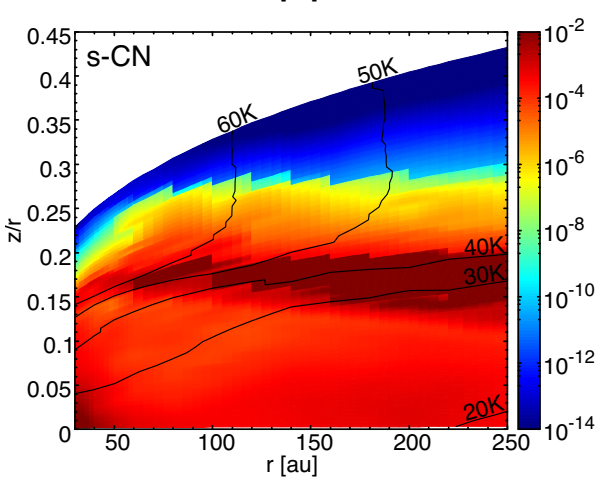

(c) M-HUV-B14

Fig. 14. Density $\left(\mathrm{cm}^{-3}\right)$ of $\mathrm{H}_{2}, \mathrm{CO}, \mathrm{CS}$ and $\mathrm{CN}$ in the gas phase of HUV-LH- $T_{\mathrm{g}}$ (left column) and of the multi-grain models in HUV regime. The black contours represent the dust temperature $\left(T_{\mathrm{d}}=T_{\mathrm{g}}\right.$ in the left column and $T_{\mathrm{d}}=T_{\mathrm{a}}$ in the middle and right columns).

We note that the channels to $\mathrm{s}-\mathrm{CH}_{3} \mathrm{SH}$ also involve successive hydrogenations (of s-CS, mainly). Reaction 13 occurs on grains of all sizes but is more effective on the large cold grains $\left(T_{\mathrm{d}} \lesssim\right.$ $15 \mathrm{~K}$ ) and Reaction 14 is only found to be effective on the colder grains.

\subsubsection{Nitrogen-bearing species: $\mathrm{NH}_{3}$}

The main nitrogen-bearing icy species in the midplane are s$\mathrm{NH}_{3}, \mathrm{~s}-\mathrm{HCN}, \mathrm{s}-\mathrm{N}_{2}$, and $\mathrm{s}-\mathrm{CN}$ at all radii. In the multi-grain models, as seen in Fig. 15, the abundance of s- $\mathrm{NH}_{3}$ is inversely proportional to the grain size until $\approx 1 \mu \mathrm{m}$, where the abundance 

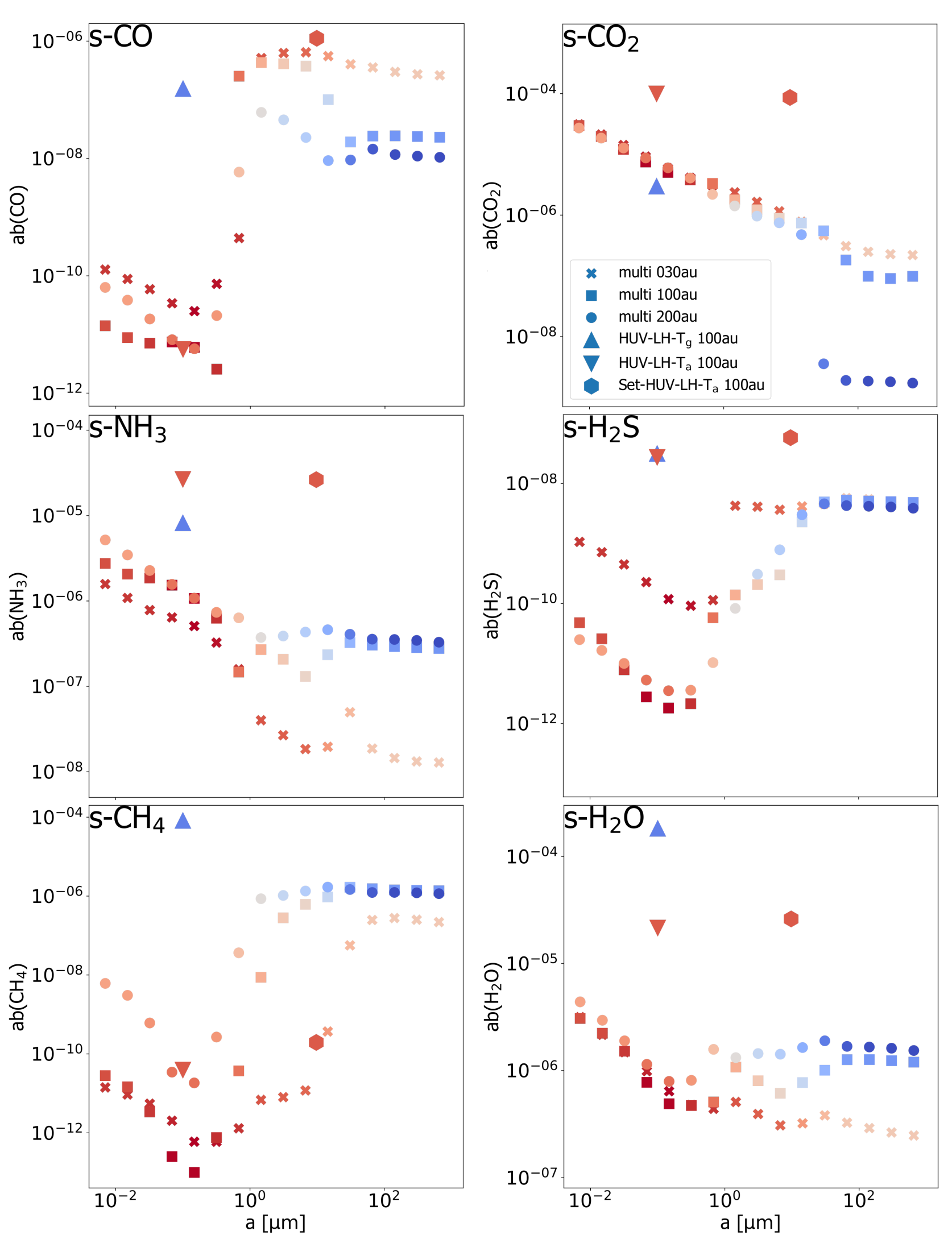

25.0

$-22.5$

20.0

17.5

15.0

12.5

10.0

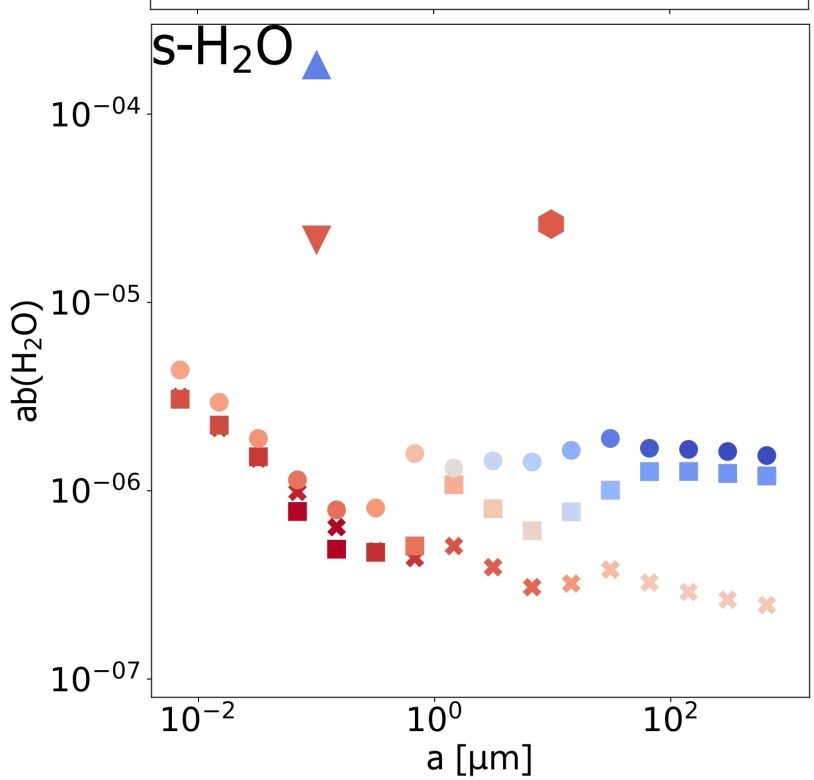

$-25.0$

$-22.5$

$-20.0$

$-17.5$

15.0

$-12.5$

10.0

Fig. 15. Grain surface abundance per total atomic hydrogen and per grain size bin for various molecules at the final stage of integration (i.e., $510^{6} \mathrm{yr}$ ) as a function of the grain radius $a$ in relation to the grain temperatures. The crosses stand for the multi-grain model at 30 au, the squares stand for the multi-grain model at $100 \mathrm{au}$, and the circles stand for $200 \mathrm{au}$. The triangles pointing upward represent the surface abundance in the single-grain model HUV-LH- $T_{\mathrm{g}}$ (grains of size $0.1 \mu \mathrm{m}$ ) and the triangle pointing downward represents the abundance in the single-grain model HUV-LH- $T_{\mathrm{a}}$, both at 100 au. Abundances being per grain size bin, the sum of the abundances in the multi-grain models should be compared to the abundances in the single-grain case. 
stops decreasing. The abundances of s- $\mathrm{NH}_{3}$ are roughly the same at 100 and 200 au but not at $30 \mathrm{au}$.

The main pathway to $\mathrm{s}-\mathrm{NH}_{3}$ originates from the accretion of $\mathrm{N}$ at the surface of the grains and its successive hydrogenations to form s- $\mathrm{NH}_{3}$ (see Aikawa et al. 2015; Eistrup et al. 2018; Ruaud \& Gorti 2019). Such hydrogenation reactions are efficient on cold surfaces $\left(T_{\mathrm{d}} \lesssim 15 \mathrm{~K}\right)$, which explains why the abundance of s- $\mathrm{NH}_{3}$ does not keep dropping when grain temperatures are low. As for the warmer small grain surfaces, their high quantity implies a high collision rate with $\mathrm{NH}_{3}$, which stays on their surface given its binding energy $\left(E_{\mathrm{b}}\left(\mathrm{NH}_{3}\right)=5500 \mathrm{~K}\right)$. As the disk evolves, a large fraction of nitrogen-bearing species are transformed into complex molecules and other routes become effective such as the destructive hydrogenation of s- $\mathrm{NH}_{2} \mathrm{CO}$ :

$\mathrm{s}-\mathrm{NH}_{2} \mathrm{CO} \stackrel{\mathrm{s}-\mathrm{H}}{\longrightarrow} \mathrm{s}-\mathrm{NH}_{3}+\mathrm{s}-\mathrm{CO}$.

\subsection{Water}

The formation of water is fundamental to explaining the evolution of protoplanetary disks and the formation of comets, which are both decisive elements to understand the delivery of water to planetary surfaces. Spectral lines of $\mathrm{H}_{2} \mathrm{O}$ in the gas phase of inner disk regions have now been widely detected in disks around T Tauri stars using near- and mid-infrared (Carr \& Najita 2008; Pontoppidan et al. 2010) or far-infrared observations (RiviereMarichalar et al. 2012). Cold water in the outer disk regions, on the other hand, is harder to detect as a consequence of low excitation lines.

It is assumed (Dominik et al. 2005; Lecar et al. 2006; Podio et al. 2013; Du \& Bergin 2014) that water on grain surfaces cannot exist inside the snowline $\left(T_{\mathrm{d}} \gtrsim 150 \mathrm{~K}\right)$, which represents radii smaller than a few to ten astronomical units around T Tauri stars. The innermost radius computed by our models is $30 \mathrm{au}$, which is beyond the radial ice line of water. Therefore, we do not discuss the radial location of the water ice line.

\subsubsection{Gas phase}

Three main parameters control the abundance of water in the gas phase, the photodesorption by FUV photons and cosmic rays, the dust temperature, and the chemical reactions in the gas phase, in particular,

$\mathrm{H}_{2}+\mathrm{OH}^{+} \rightarrow \mathrm{H}+\mathrm{H}_{2} \mathrm{O}^{+}$

$\mathrm{H}_{2} \mathrm{O}^{+}+\mathrm{H}_{2} \rightarrow \mathrm{H}+\mathrm{H}_{3} \mathrm{O}^{+}$

$\mathrm{H}_{3} \mathrm{O}^{+}+\mathrm{e}^{-} \rightarrow \mathrm{H}+\mathrm{H}_{2} \mathrm{O}$

Thus $\mathrm{H}_{2}$ and $\mathrm{OH}^{+}$are the main precursors for $\mathrm{H}_{2} \mathrm{O}$. Whether $\mathrm{H}_{2}$ or $\mathrm{OH}^{+}$is the limiting factor is determined by another sequence as follows:

$\mathrm{H}_{2}+\mathrm{CR} \rightarrow \mathrm{H}_{2}^{+}+\mathrm{e}^{-}$
$\mathrm{H}_{2}^{+}+\mathrm{H}_{2} \rightarrow \mathrm{H}_{3}^{+}+\mathrm{H}$
$\mathrm{H}_{3}^{+}+\mathrm{O} \rightarrow \mathrm{OH}^{+}+\mathrm{H}_{2}$$$
\mathrm{H}_{3}^{+}+\mathrm{O} \rightarrow \mathrm{OH}^{+}+\mathrm{H}_{2}
$$

These two sequences show that $\mathrm{H}_{2} \mathrm{O}$ formation depends on $\mathrm{H}_{2}$ and $\mathrm{OH}^{+}$but the latter also depends on $\mathrm{H}_{2}$. Therefore, this is the $\mathrm{H}_{2}$ abundance that sets the gas-phase formation of water and accounts for the difference between the models (not cosmic-rays or $\mathrm{OH}^{+}$). However, the gas-phase formation of $\mathrm{H}_{2} \mathrm{O}$ only represents a small fraction of all $\mathrm{H}_{2} \mathrm{O}$ molecules formed as most $\mathrm{H}_{2} \mathrm{O}$ are formed on grain surfaces. The abundance of gas-phase water is thus mainly governed by the formation rate of icy water and by the photodesorption rate. Thermal desorption is ineffective in most parts of the disk because the binding energy of water is high. The formation rate depends on the grain temperature, while the photodesorption rate depends on the FUV flux and cosmic rays. The analysis of the results herein (Figs. 16 and 17) shows that the dust temperature plays a major role in the production of gas-phase water.

Single-grain models. The vertical snowline forms a clear boundary between high- and low gas-phase $\mathrm{H}_{2} \mathrm{O}$ abundance at $z / r \sim 0.15$ (Fig. 16) and we see that HUV-B14- $T_{\text {a }}$ produces the most distinct boundary of all three models. The snowline corresponds roughly to the abrupt drop in the $u v$ flux as shown in Fig. 2 (bottom-right panel). The cold-grain model HUVLH- $T_{\mathrm{g}}$ produces substantially more water in the upper layers than the other single-grain models because low dust temperature increases the hydrogenation rates on the grain surfaces. The photodesorption rates are approximately equal in the three models (same flux) but since HUV-LH- $T_{\mathrm{g}}$ produces a larger water abundance on grain surfaces the final gas-phase abundance is much larger. It results in a column density of water about a factor 10 larger in HUV-LH- $T_{\mathrm{g}}$ than in the other two models. On the other hand, the $\mathrm{B} 14$ prescription increases only marginally the column density of gas-phase water.

Multi-grain models. The vertical snowline is far less apparent (Fig. 17, top row, middle and right column). Both models have approximately the same distribution of abundance because they both have the same dust temperature distribution and $u v$. We note, however, that the B14 prescription in M-HUV-B14 produces marginally more water in the upper layer of the disk than in M-HUV-LH owing to the gas-phase formation of water via the two sequences presented above.

\subsubsection{Water ice}

Single-grain models. The vertical snowline location is clearly defined (see Fig. 16, bottom row), although we note the presence of a notable band around $z=3 H(z=2 H)$ in HUVLH- $T_{\mathrm{g}}$ (resp. HUV-LH- $T_{\mathrm{a}}$ ). This is because of the following activated hydrogenation reaction:

$\mathrm{s}-\mathrm{H}+\mathrm{s}-\mathrm{HOOH} \rightarrow \mathrm{s}-\mathrm{H}_{2} \mathrm{O}+\mathrm{s}-\mathrm{OH}\left(\mathrm{E}_{\mathrm{A}}=1400 \mathrm{~K}\right)$.

The band illustrates the location in the disk where this reaction is activated. Below the band, the reaction is not activated and water ice is mostly photodesorbed, while above the band the photodesorption rates competes with the formation rate and the abundance drops. In HUV-LH- $T_{\mathrm{a}}$ (Fig. 16, middle column), Reaction 22 is activated at smaller altitudes in the disk since the dust temperature is globally higher, resulting in a peak in abundance at around 2 scale heights. In contrast, HUV-B14- $T_{\text {a }}$ does not exhibit such a peak near $z=2 H$ since a large fraction of atomic hydrogen on surfaces is used to create molecular hydrogen. This dramatically decreases the quantity of available $\mathrm{H}$ atoms necessary to form water.

Multi-grain models. The same mechanisms are at work (see middle and right columns of Fig. 17). Yet, the dust temperature distribution involves that we find hot (up to $\sim 95 \mathrm{~K}$ ) and cold (as low as $\sim 7 \mathrm{~K}$ ) grains nearly everywhere in the disk. Consequently, Reaction 22 is activated at lower altitudes and the formation of s$\mathrm{H}_{2} \mathrm{O}$ through hydrogenation can occur at higher altitudes on cold medium- and large-sized grains so that the snowline is located at a higher altitude. 

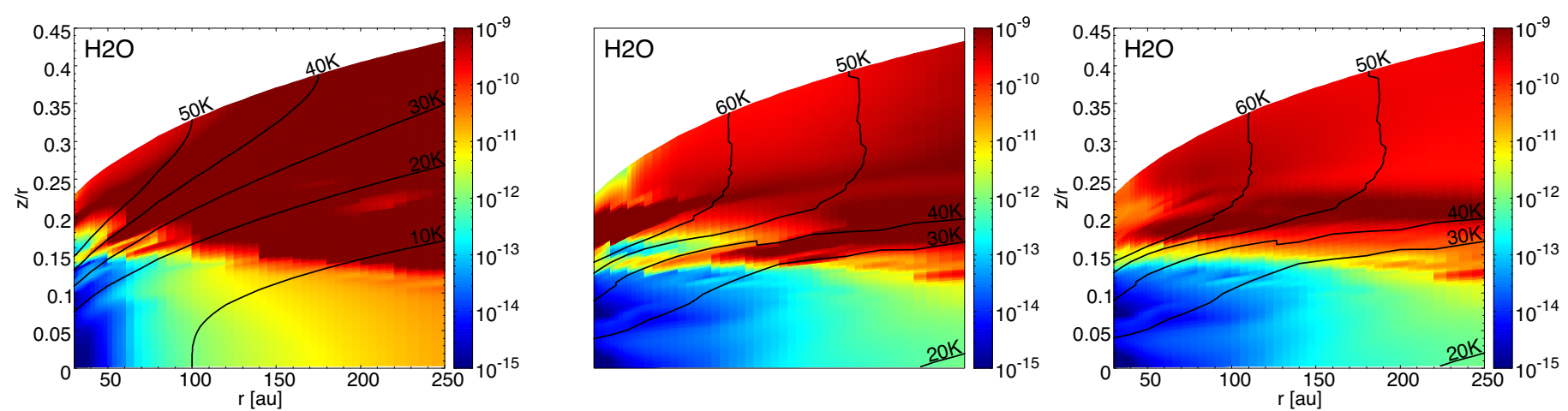

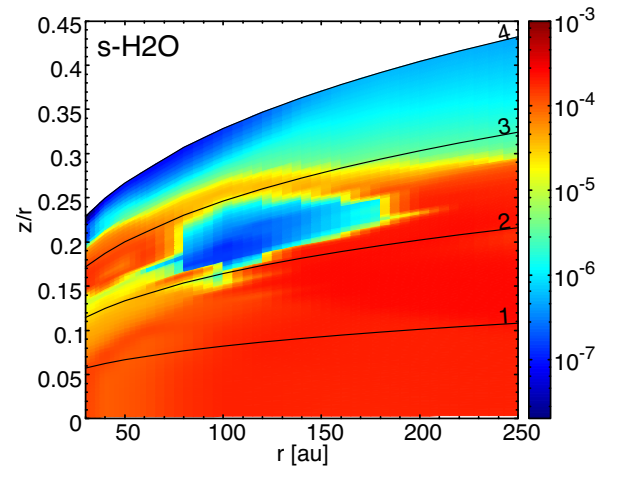

(a) HUV-LH-T ${ }_{g}$

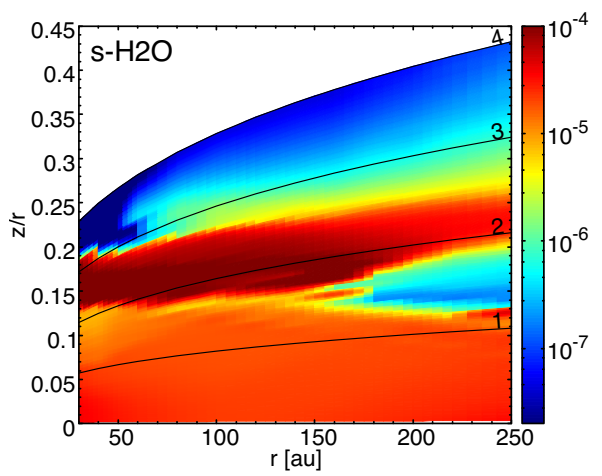

(b) HUV-LH-T

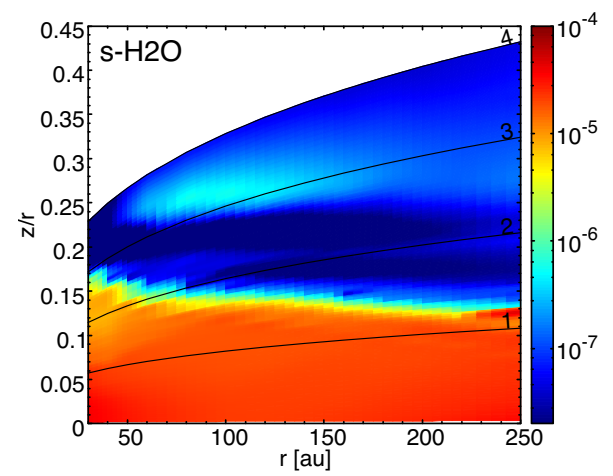

(c) HUV-B14-T

Fig. 16. Water abundance in the gas phase (top) and on the grain surfaces (bottom) of the single-grain models. The left column shows the abundances in HUV-LH- $T_{\mathrm{g}}$, middle in HUV-LH- $T_{\mathrm{a}}$, and right in HUV-B14- $T_{\mathrm{a}}$. In the top row, the solid black lines show the dust temperature isocontours; in the bottom row they denote isocontours of scale heights.
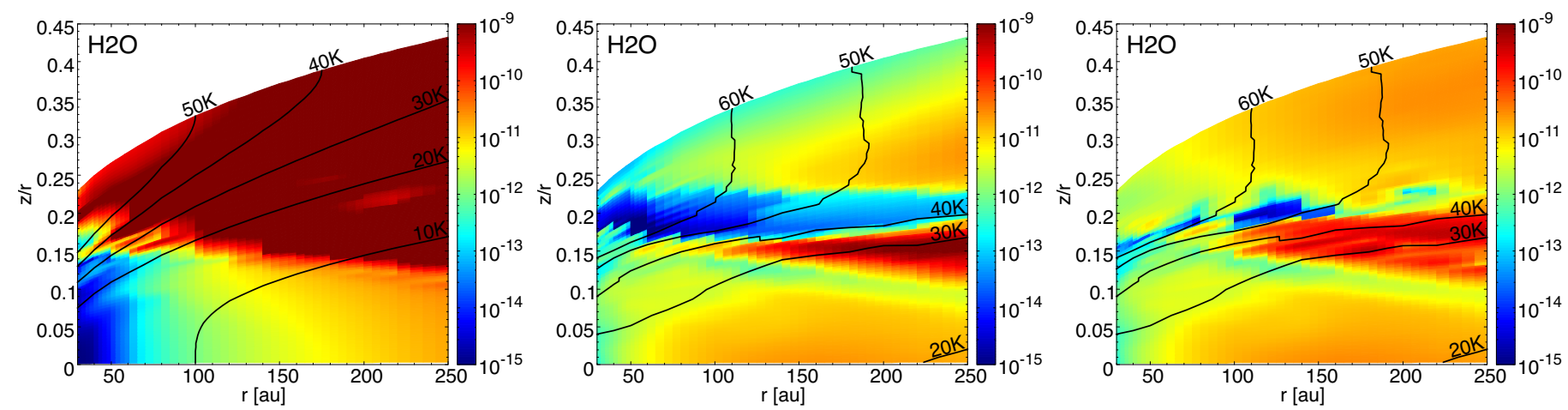

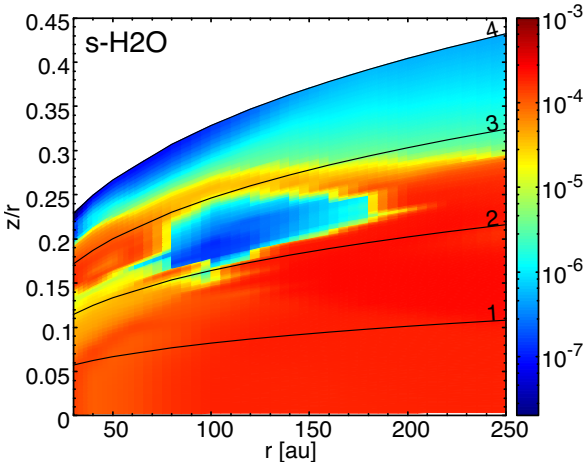

(a) HUV-LH-T $g$

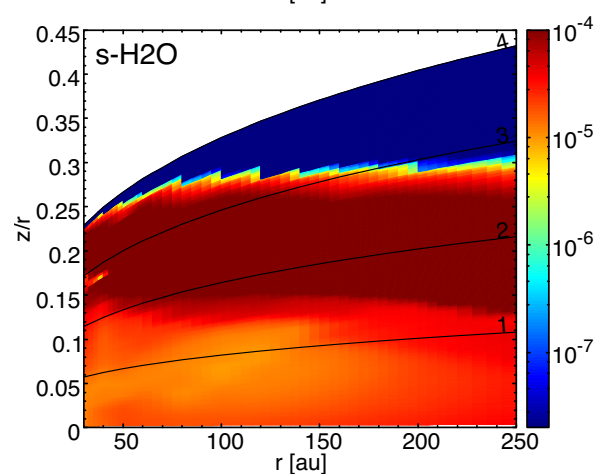

(b) M-HUV-LH

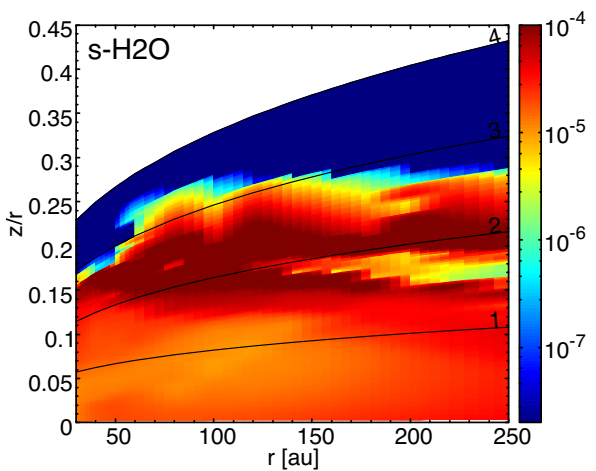

(c) M-HUV-B14

Fig. 17. Water abundance in the gas phase (top) and on the grain surfaces (bottom). The left column shows the abundances in HUV-LH- $T_{\mathrm{g}}$ and the middle and right column in the multi-grain models. In the top row, the solid black lines show the dust temperature isocontours; in the bottom row they denote isocontours of scale heights. 
Around the midplane. In all models, water is found in the form of ice around the midplane, where the desorption by $u v$ flux and cosmic-rays is limited and where hydrogenation is effective. For single-grain models, the width of the region where water is on the icy mantles is larger in HUV-LH- $T_{\mathrm{g}}$ than in the two others because, again, the dust temperature is smaller. As presented in Fig. 15 (bottom right panel), the abundance is roughly inversely proportional to the grain size at $30 \mathrm{au}$. The reason is the combination of the large binding energy of water and the high density of small grains. At 100 and $200 \mathrm{au}$, however, the abundance on large grains (size $>0.1 \mu \mathrm{m}$ ) is larger because the grain temperature is low enough to allow for effective hydrogenation.

\subsection{COMs}

Complex organic molecules (COMs), supposedly constituting the crucial bond between simple ISM molecules and prebiotic chemistry, are hard to detect as a consequence of their low abundance and weak emission lines originating from their molecular complexity. This is especially true for objects with small spatial extensions such as disks. Simple organic molecules have been regularly detected over the last decades such as $\mathrm{H}_{2} \mathrm{CO}$ (Dutrey et al. 1997; Aikawa et al. 2003; Öberg et al. 2011), $\mathrm{HC}_{3} \mathrm{~N}$ (Chapillon et al. 2012), $\mathrm{CH}_{3} \mathrm{CN}$ (Öberg et al. 2015), and $\mathrm{CH}_{3} \mathrm{OH}$ (Walsh et al. 2016). We investigate the effectiveness of our models in synthesizing COMs from surfaces or the gas phase when given sufficient time $\left(5 \times 10^{6} \mathrm{yr}\right)$.

\subsubsection{Methyl cyanide $\left(\mathrm{CH}_{3} \mathrm{CN}\right)$}

$\mathrm{C}-\mathrm{N}$ bonded species are very important for the synthesis of glycine (Goldman et al. 2010). Öberg et al. (2015) have observed methyl cyanide $\left(\mathrm{CH}_{3} \mathrm{CN}\right)$ in the disk around the Herbig Ae star MWC 480, a star exhibiting a stronger $u v$ flux and a warmer disk than a T Tauri star.

$\mathrm{CH}_{3} \mathrm{CN}$ to $\mathrm{HCN}$ abundance ratio. To facilitate comparisons, we discuss the abundance of $\mathrm{CH}_{3} \mathrm{CN}$ relative to $\mathrm{HCN}$, as practiced in cometary studies (Mumma \& Charnley 2011; Öberg et al. 2015). The model that produces the largest $\mathrm{CH}_{3} \mathrm{CN} / \mathrm{HCN}$ ratio is the single-grain model HUV-LH- $T_{\mathrm{g}}$ with $\sim 14 \%$ (vertically integrated) at 100,120 , and $140 \mathrm{au}$, while the other single-grain models produce nearly more than $\sim 1 \%$ at these radii. The outer regions (200-250 au) produce a larger ratio (up to $9 \%$ ) than the inner disk regions in the warm grain singlemodels, whereas the peak is in the region around 100 au in HUV-LH- $T_{\mathrm{g}}$. In multi-grain models, the gas-phase abundance ratio never exceeds $1 \%$. However, it never goes below $0.1 \%$, implying a rather uniform production of $\mathrm{CH}_{3} \mathrm{CN}$ along the radius as compared to the single-grain models. M-HUV-B14 produces slightly more $\mathrm{CH}_{3} \mathrm{CN}$ than M-HUV-LH. The model HUV-LH$T_{\mathrm{g}}$ (with the coldest grains overall) globally produces the largest $\mathrm{CH}_{3} \mathrm{CN} / \mathrm{HCN}$ ratio.

$\mathrm{CH}_{3} \mathrm{CN}$ in gas phase. In the upper regions, the main known pathways to form gas-phase $\mathrm{CH}_{3} \mathrm{CN}$ are the reaction of $\mathrm{HCN}$ with $\mathrm{CH}_{3}^{+}$and the photodesorption of icy $\mathrm{CH}_{3} \mathrm{CN}$; thermal desorption is not effective because of the large binding energy of $\mathrm{CH}_{3} \mathrm{CN}$. Results show that the reaction between $\mathrm{HCN}$ and $\mathrm{CH}_{3}^{+}$ is the most effective route in M-HUV-B14, while only photodesorption is effective in M-HUV-LH. The reason for this is straightforward since the production of $\mathrm{CH}_{3}^{+}$requires the

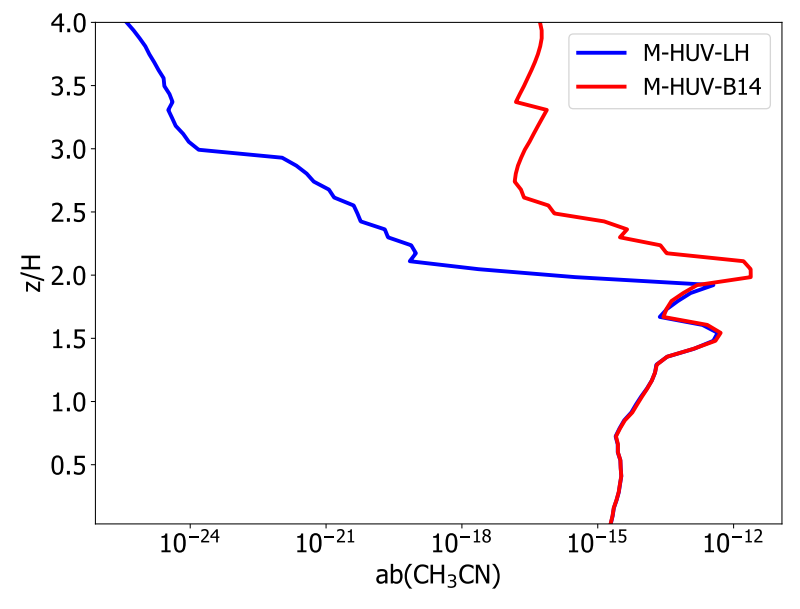

Fig. 18. Altitude above the midplane as a function of the abundance relative to $\mathrm{H}$ of $\mathrm{CH}_{3} \mathrm{CN}$ at $100 \mathrm{au}$. The blue line shows the abundance produced by model M-HUV-LH; the red line shows the abundance produced by model M-HUV-B14. The abundance dramatically drops above $\sim 2$ scale heights in M-HUV-LH, while M-HUV-B14 keeps a rather high abundance at high altitudes. This is due to the available quantity of gas-phase $\mathrm{H}_{2}$ in M-HUV-B14, which enhances the production of $\mathrm{CH}_{3}^{+}$.

presence of molecular hydrogen as follows:

$\mathrm{C}^{+} \stackrel{\mathrm{H}_{2}}{\longrightarrow} \mathrm{CH}_{2}^{+} \stackrel{\mathrm{H}_{2}}{\longrightarrow} \mathrm{H}+\mathrm{CH}_{3}^{+}$

and $\mathrm{H}_{2}$ is much more abundant in M-HUV-B14 in the upper layers. At $100 \mathrm{au}$, the abundance of $\mathrm{CH}_{3}^{+}$in M-HUV-LH is very low $\left(\sim 10^{-20}\right)$ as compared to the abundance in M-HUV-B14 $\left(\sim 10^{-10}\right)$. It follows that, as seen in Fig. 18, M-HUV-B14 produces much more $\mathrm{CH}_{3} \mathrm{CN}$ than in M-HUV-LH. Therefore, the presence of $\mathrm{H}_{2}$ appears to be decisive for the production of gasphase $\mathrm{CH}_{3} \mathrm{CN}$ in the upper regions of the disk. However, this difference between the two models does not significantly impact the total column density of $\mathrm{CH}_{3} \mathrm{CN}$ as most of it is produced near the midplane.

$\mathrm{CH}_{3} \mathrm{CN}$ ices. Figure 19 (bottom) shows the abundance of s- $\mathrm{CH}_{3} \mathrm{CN}$ ices in the midplane relative to the grain size as a function of the radius. $\mathrm{s}-\mathrm{CH}_{3} \mathrm{CN}$ mostly stays on warm small grains while there is a large drop in abundance around grain populations of size $\sim 0.317 \mu \mathrm{m}$. The results show that the two main reactions that lead to s- $\mathrm{CH}_{3} \mathrm{CN}$ are the adsorption of gas-phase $\mathrm{CH}_{3} \mathrm{CN}$ onto the surfaces and the following surface reaction:

$\mathrm{s}-\mathrm{CH}_{3}+\mathrm{s}-\mathrm{CN} \rightarrow \mathrm{s}-\mathrm{CH}_{3} \mathrm{CN}$.

$\mathrm{s}-\mathrm{CH}_{3} \mathrm{CN}$ is more abundant on small grains for three main reasons. The first reason is the large number density of small grains that implies large collision rates with gas-phase produced $\mathrm{CH}_{3} \mathrm{CN}$ and with $\mathrm{CH}_{3}$ that, despite a low binding energy, briefly sticks and quickly reacts with s-CN (see reaction 24). The second reason is the high binding energy of $\mathrm{CH}_{3} \mathrm{CN}$ $\left(\mathrm{E}_{\mathrm{b}}\left(\mathrm{CH}_{3} \mathrm{CN}\right)=4680 \mathrm{~K}\right.$ Wakelam et al. 2017) and of the precursors (s-CN) so that they stay on small grain surfaces. Moreover, the temperature of small grains is sufficiently high to allow precursors to diffuse on the surface and increase reaction rates. The last reason is that even if s- $\mathrm{CH}_{3} \mathrm{CN}$ ices can be formed on large grain surfaces, photodesorption rates are large enough for $\mathrm{CH}_{3} \mathrm{CN}$ to be desorbed from large grains and seed small grains as the disk evolves. 


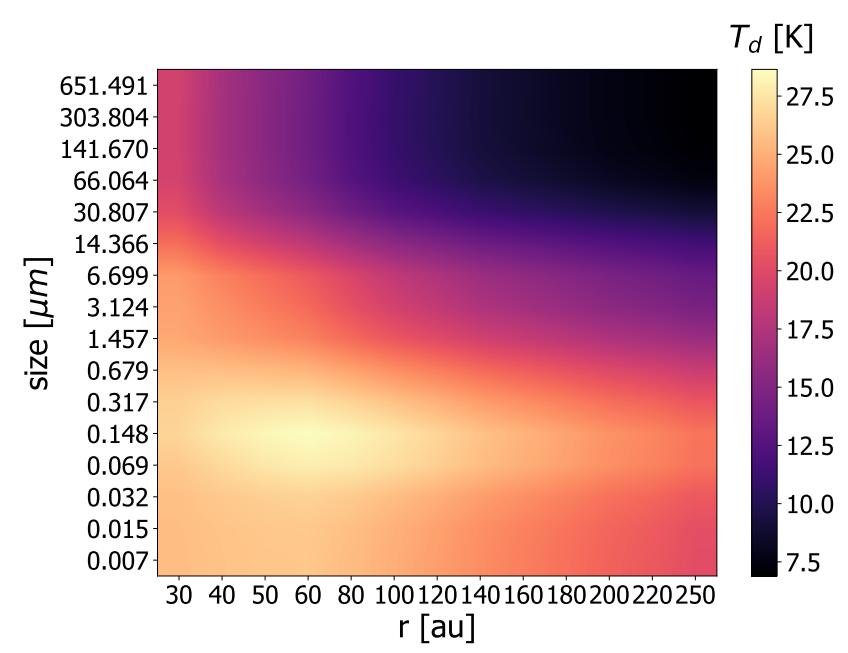

$\mathrm{ab}\left(\mathrm{s}-\mathrm{H}_{2} \mathrm{CO}\right)$

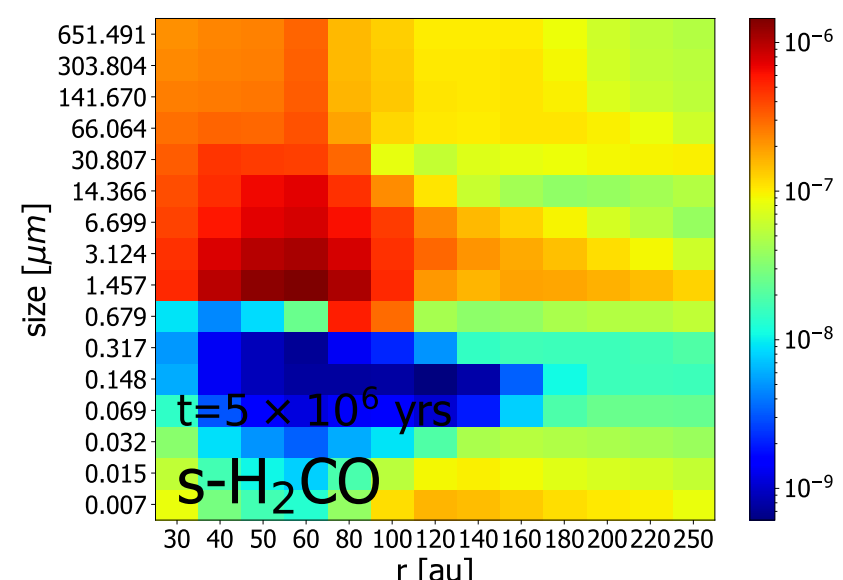

$\mathrm{ab}\left(\mathrm{s}-\mathrm{CH}_{3} \mathrm{CN}\right)$

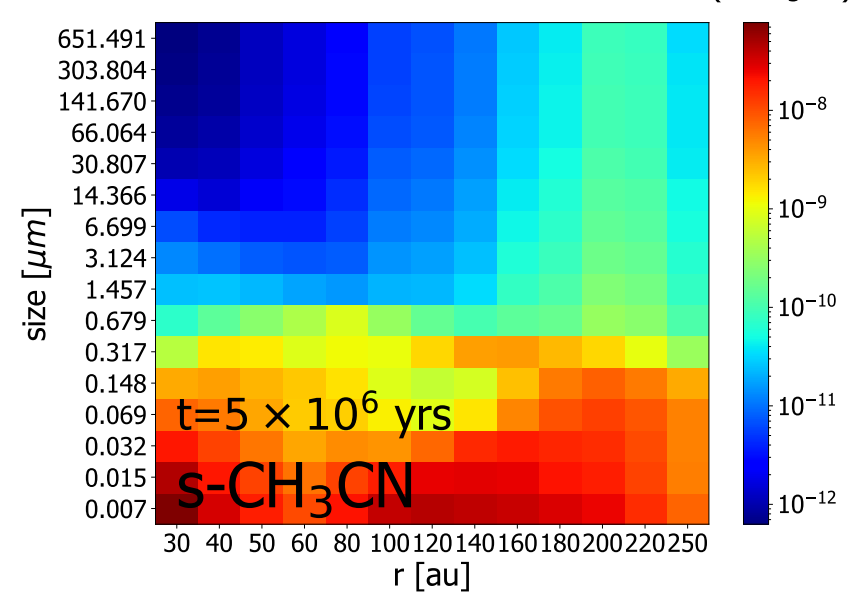

$\mathrm{ab}(\mathrm{s}-\mathrm{CO})$

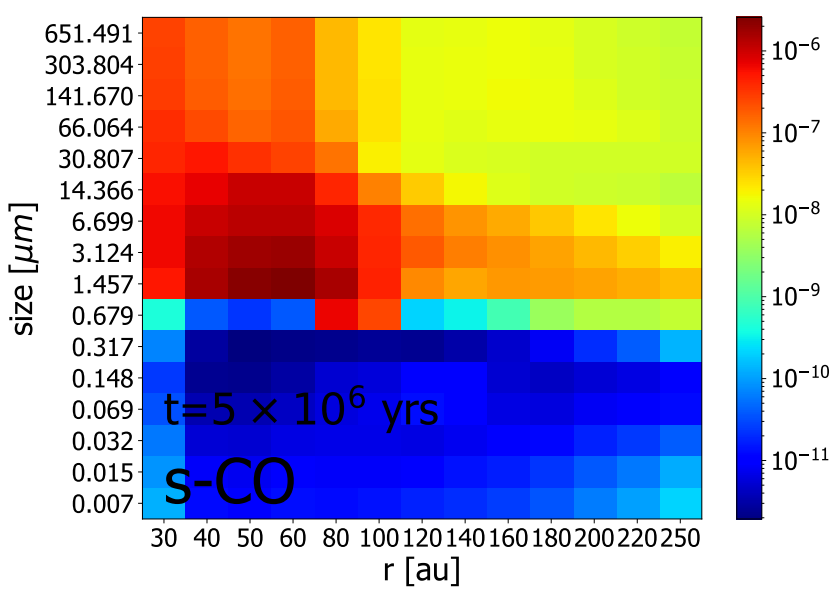

$\mathrm{ab}\left(\mathrm{s}-\mathrm{CH}_{3} \mathrm{OH}\right)$

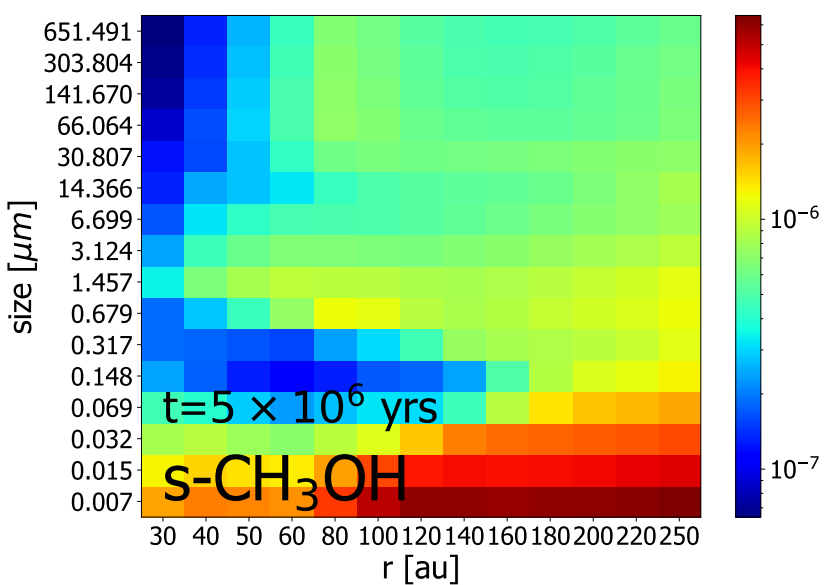

Fig. 19. Multi-grain models: maps of ice abundance relative to $\mathrm{H}$ in the midplane, with grain sizes as a function of the radius. The top left panel is the distribution in grain temperature. The hottest region is located at around $60 \mathrm{au}$ from the star on grains of size $0.148 \mu \mathrm{m}$.

\subsubsection{Methanol and formaldehyde}

$\mathrm{CH}_{3} \mathrm{OH}$ in gas-phase. For multi-grain models, Fig. 20 shows that the vertical profile of gas-phase $\mathrm{CH}_{3} \mathrm{OH}$ is divided into two layers separated by an abrupt drop in abundance at $\sim 2 \mathrm{H}$, which corresponds roughly to the dust temperature transition (Fig. 5). There is a peak in gas-phase $\mathrm{CH}_{3} \mathrm{OH}$ at $\sim 1.8 \mathrm{H}$ in both models. This corresponds to the location at which the dust temperature is low enough for adsorbed $\mathrm{CO}$ to be hydrogenated and at which the $u v$ flux is high enough to efficiently photodissociate a significant fraction of newly formed methanol. The lower layer $(<2 \mathrm{H})$ contains most of $\mathrm{CH}_{3} \mathrm{OH}$ column density. In the upper layer, M-HUV-B14 produces more $\mathrm{CH}_{3} \mathrm{OH}$ than M-HUV-LH.

$\mathrm{CH}_{3} \mathrm{OH}$ to $\mathrm{H}_{2} \mathrm{CO}$ abundance ratio. In multi-grain models, the ratio $\mathrm{CH}_{3} \mathrm{OH} / \mathrm{H}_{2} \mathrm{CO}$ increases by a factor of $\sim 2-3$ in the outermost region ( $\geq 200 \mathrm{au}$ ) between $10^{6}$ and $5 \times 10^{6} \mathrm{yr}$, while 


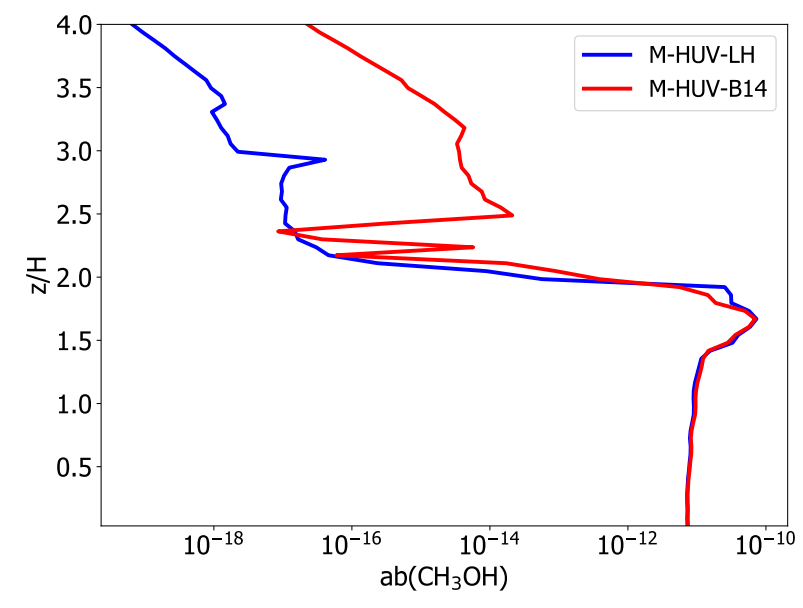

Fig. 20. Altitude above the midplane as a function of the abundance relative to $\mathrm{H}$ of $\mathrm{CH}_{3} \mathrm{OH}$ at $100 \mathrm{au}$. The blue line shows the abundance produced by model M-HUV-LH; the red line shows the abundance produced by model M-HUV-B14. The abundance dramatically drops at $\sim 2$ scale heights in both models, although M-HUV-B14 maintains a larger abundance than M-HUV-LH at high altitudes.

it remains stable in the inner region (<100 au). In M-HUV-B14, after $10^{6} \mathrm{yr}$, the averaged ratio in the region $80-120$ au is equal to 1.3 . This appears to be consistent with the observed ratio in TW Hya (Walsh et al. 2016; Carney et al. 2019). In M-HUV$\mathrm{LH}$, the averaged ratio is equal to 2.4 . The difference is due to a more efficient pathway to form $\mathrm{H}_{2} \mathrm{CO}$ in the upper disk regions of M-HUV-B14. Gas-phase $\mathrm{H}_{2} \mathrm{CO}$ is efficiently formed through the following sequence:

$\mathrm{H}_{2}+\mathrm{CH}_{3}^{+} \rightarrow \mathrm{CH}_{5}+$

$\mathrm{CH}_{5}^{+}+\mathrm{e}^{-} \rightarrow \mathrm{H}+\mathrm{H}+\mathrm{CH}_{3} / \mathrm{H}_{2}+\mathrm{CH}_{3}$

$\mathrm{O}+\mathrm{CH}_{3} \rightarrow \mathrm{H}+\mathrm{H}_{2} \mathrm{CO}$

This shows that molecular hydrogen is a key element in the formation process of gas-phase formaldehyde and M-HUV-B14 provides much more $\mathrm{H}_{2}$ than M-HUV-LH.

Grain size dependence of surface chemistry for $\mathrm{CH}_{3} \mathrm{OH}$. Figure 19 gives a set of maps of ice abundance in the midplane with grain sizes as a function of the computed radii. The top left panel is the grain temperature that allows us to analyze the surface abundance in view of the surface temperature. We have same results for both multi-grain models. On icy mantles, $\mathrm{s}-\mathrm{H}_{2} \mathrm{CO}$ and $\mathrm{s}-\mathrm{CH}_{3} \mathrm{OH}$ are formed via the same hydrogenation sequence as follows:

$\mathrm{s}-\mathrm{CO} \stackrel{\mathrm{H}}{\rightarrow} \mathrm{s}-\mathrm{HCO} \stackrel{\mathrm{H}}{\rightarrow} \mathrm{s}-\mathrm{H}_{2} \mathrm{CO} \stackrel{\mathrm{H}}{\rightarrow} \mathrm{s}-\mathrm{CH}_{3} \mathrm{O} \stackrel{\mathrm{H}}{\rightarrow} \mathrm{s}-\mathrm{CH}_{3} \mathrm{OH}$.

However, we note that $\mathrm{H}_{2} \mathrm{CO}$ is mainly formed in the gas phase. As seen in Fig. 19 (top right), CO tends to be efficiently adsorbed onto cold surfaces $\left(T_{\mathrm{d}} \lesssim 17 \mathrm{~K}\right)$. The map of $\mathrm{H}_{2} \mathrm{CO}$ (bottom left) grossly follows the same distribution although the trend is globally less pronounced. $\mathrm{CH}_{3} \mathrm{OH}$, on the other hand, exhibits a totally different distribution and tends to concentrate on small grain surfaces (bottom right). The main reason comes from the fact that the binding energy increases as the molecules become more complex (e.g., $E_{\mathrm{b}}(\mathrm{CO}) \sim 1200 \mathrm{~K}, E_{\mathrm{b}}\left(\mathrm{H}_{2} \mathrm{CO}\right) \sim 3200 \mathrm{~K}$, $E_{\mathrm{b}}\left(\mathrm{CH}_{3} \mathrm{OH}\right) \sim 5500 \mathrm{~K}$ Minissale et al. 2016; Noble et al. 2012; Collings et al. 2004) and that the hydrogenation of s- $\mathrm{H}_{2} \mathrm{CO}$ (which leads to $\mathrm{s}-\mathrm{CH}_{3} \mathrm{OH}$ ) has an activation barrier and cannot efficiently occur on cold grain surfaces.

\subsection{Impact for planet formation}

Planet formation is strongly linked to grain growth and dust disk evolution. Our study reveals some interesting trends that should influence planetary formation.

For a T Tauri disk of a few Myr, radial changes of the millimeter spectral index (e.g., Birnstiel et al. 2010) reveal that, after settling, large grains have drifted toward the central star. Typically, grains larger than a few $0.1-1 \mathrm{~mm}$ are located inside a radius of 50-80 au. Beyond this, dust disks have a spectral index that is similar to that observed in the ISM, suggesting a similar size distribution, even if settling still occurs with larger grains residing onto the midplane. As a consequence, toward the midplane, we expect to observe different icy layers on grains in the inner (large cold grains) and outer disk (warmer smaller grains). We have seen in the previous section that $\mathrm{s}-\mathrm{CH}_{4}$ and $\mathrm{s}-\mathrm{H}_{2} \mathrm{O}$ are preferentially located on large grains while s- $\mathrm{CO}_{2}$ remains on smaller grains (Fig. 15). This also implies a different chemical coupling between the gas and solid phase. Moreover, we have seen that complex molecules tend to stick and remain on small warmer grains $(\leq \sim 0.1 \mu \mathrm{m})$, which are less likely to drift toward the inner disk. The outer disk ( $r \geq 80 \mathrm{au}$ ) is therefore assumed to support rich chemistry on small grains, which are probable precursors to cometary nuclei. On the other hand, as larger grains drift efficiently toward the inner region, their surface temperature is likely to vary during the journey, which can allow for the growing chemical complexity to occur until the less refractory molecules become vaporized when sufficiently close to the star. Therefore, rich chemistry of the inner disk region can be assumed to come from the gas phase, which is later accreted by planetary embryos, while rich chemistry of the outer disk region occurs on the small grain surfaces and then seeds the inner region through planet-comet collisions in later phases of the disk.

Grain-grain collisions may modify this picture. The characteristic timescale (the time for a large grain to encounter small grains of equivalent area) around $100 \mathrm{au}$ is on the order of $10^{4}-$ $10^{5} \mathrm{yr}$. However, while large grains may accrete the smaller grains, and inherit part of their surface composition while growing, small grains are recreated by disruptive events that may severely affect their surface layer and ice mantles. The outcome of the surface state during such grain-grain collisions remains to be studied.

Nevertheless, in all cases, the grain size distribution, because it results in a spread of dust temperature, allows for a faster depletion of $\mathrm{C}$ and $\mathrm{O}$, which are converted to $\mathrm{s}-\mathrm{CO}_{2}$ on the colder grains in the disk midplane, but also a more active surface chemistry leading to formation of COMs on the warmer grains in the upper layer. Such a behavior cannot be reproduced by a single-grain temperature chemical model because the formation processes are highly nonlinear, since the mobility and evaporation efficiency depend exponentially on dust temperature.

The range in dust temperatures also spatially spreads out the snowlines that become fuzzy. The spatial extent of these "snow bands" depends on the specific dust properties and disk mass. The temperature spread is larger for less massive disks.

As disks evolve, the amount of dust (and gas) decreases until they reach the optically thin, debris disk phase. A less massive dust disk necessarily implies a warmer dust disk, which should affect the composition of the icy layers at the grain surface in evolved disks. Recent surveys of 5-40 Myr old disks suggest the existence of an intermediate phase (at least for Herbig Ae stars) between debris and protoplanetary disks (e.g., Kóspál et al. 2013; 
Péricaud et al. 2017). The so-called hybrid disks share dust properties similar to those observed around debris disks, while the amount of gas is still significant, and a larger than standard gasto-dust-ratio is observed. For instance, in HD141569, Di Folco et al. (2020) observed a CO surface density that is only a factor 10 lower than in a typical protoplanetary HAe disk, whereas the amount of millimeter grains is a factor 50 times smaller than in typical young disks. The reduced amount of grains, and in some cases at least possible changes in the grain size distribution (e.g., Hughes et al. 2018), should affect the grain surface chemistry during the late phases of disk evolution. More simulations of older, gas-rich disks with a small amount of dust and a larger gas-to-dust ratio are needed to quantify the impact on chemistry and planet formation.

\section{Summary}

In this work we presented a new chemical model of protoplanetary disks that consistently accounts for the impact of grain sizes on the dust temperatures and $u v$ penetration. Representative results were obtained for a typical disk around T Tauri stars, using parameters derived from the Flying Saucer observations (Dutrey et al. 2017). We coupled the chemical code NAUTILUS and radiative transfer code POLARIS to use a consistent distribution of grain sizes and temperatures, accounting for sizedependent dust settling. We implemented in NAUTILUS a new method to account for self and mutual shielding of molecules in photo processes, which accounts for the frequency dependence of photorates. The outputs of multi-grain models are compared to those of single-grain size, using two different assumptions for the single-grain temperature: equal to the (assumed) gas temperature or to the area-weighted mean temperature of the multi-grain models.

- We identify the formation of $\mathrm{H}_{2}$ through the LH mechanism as inefficient for a grain size distribution and the amount of atomic $\mathrm{H}$ as critical for the chemistry of the upper layers $(2-4 H)$. The parametric method of Bron et al. (2014), which accounts for temperature fluctuations for small grains, appears more appropriate to produce enough $\mathrm{H}_{2}$ :

- Comparisons between the models reveal differences of more than one to two orders of magnitudes for some abundant gas-phase species. Most of these differences arise from above $1.5 \mathrm{H}$, despite the larger $u v$ penetration in multi-grain models;

- The location of the $\mathrm{H}_{2} \mathrm{O}$ vertical snowline is partly regulated by the $u v$ penetration but also by the amount of $\mathrm{H}_{2}$. In the upper disk layers, the amount of $\mathrm{H}_{2}$ also regulates the formation of $\mathrm{H}_{2} \mathrm{CO}$, although this molecule is mostly formed, like $\mathrm{CH}_{3} \mathrm{OH}$, on grain surfaces;

- $\mathrm{CH}_{3} \mathrm{CN}$, like $\mathrm{CH}_{3} \mathrm{OH}$ to a lesser extent, is preferentially formed on warmer grains that allow for a larger diffusivity of precursors on the grain surface;

- At $100 \mathrm{au}$, in the midplane, disks with colder dust produce more $\mathrm{H}_{2} \mathrm{O}$ and $\mathrm{CH}_{4}$, while those with warmer dust produce more $\mathrm{CO}_{2}$ on grain surfaces;

- As soon as CO is locked onto large (hence colder) grains $(a>1 \mu \mathrm{m})$, it remains trapped until the end of the simulated evolution;

- The spread of temperatures available with grain size distributions simultaneously provides surface chemistry for cold and warm grains that cannot be mimicked by a unique dust temperature. In particular, this allows depletion of $\mathrm{C}$ and $\mathrm{O}$ to proceed efficiently (by conversion to $\mathrm{CO}_{2}$ or $\mathrm{CH}_{4}$ and $\mathrm{H}_{2} \mathrm{O}$ on colder grains), while the COMs production is boosted on the warmer grains;

- Using a single-grain size that locally mimicks grain growth and dust settling fails to reproduce the complex gas-grain chemistry resulting from grain temperature depending on grain size;

- Another direct consequence of having different dust temperatures at the same location is a spatial spread out of the snowlines. High angular observations would be required to test this prediction.

Our results clearly show that the chemistry of protoplanetary disks cannot simply be represented by a single-grain size and temperature. Finally, our disk model assumes a relatively high dust mass (i.e., an optically thick dust disk). An optically thinner dust disk would impact the $u v$ penetration and dust temperature changing the chemistry at play even on the midplane. This remains to be investigated to determine how it can affect the grain surfaces and then the composition of planetesimals and embryos.

Acknowledgements. We thank E. Bron for fruitful discussions about the $\mathrm{H}_{2}$ formation in PDRs and M. Ruaud for a detailed reading of the article. We also thank an anonymous referee who helped to improve the paper. This work was supported by the "Programme National de Physique Chimie du Milieu Interstellaire" (PCMI) from INSU/CNRS. J.K. acknowledges support from the DFG grants WO 857/13-1, WO 857/15-1, and WO 857/17-1.

\section{References}

Acharyya, K., Hassel, G. E., \& Herbst, E. 2011, ApJ, 732, 73 Agúndez, M., Roueff, E., Le Petit, F., \& Le Bourlot, J. 2018, A\&A, 616, A19 Aikawa, Y., \& Nomura, H. 2006, ApJ, 642, 1152

Aikawa, Y., Momose, M., Thi, W.-F., et al. 2003, PASJ, 55, 11

Aikawa, Y., Furuya, K., Nomura, H., \& Qi, C. 2015, ApJ, 807, 120

Akimkin, V., Zhukovska, S., Wiebe, D., et al. 2013, ApJ, 766, 8

Birnstiel, T., Dullemond, C. P., \& Brauer, F. 2010, A\&A, 513, A79

Boehler, Y., Dutrey, A., Guilloteau, S., \& Piétu, V. 2013, MNRAS, 431, 1573

Bron, E., Le Bourlot, J., \& Le Petit, F. 2014, A\&A, 569, A100

Carney, M. T., Hogerheijde, M. R., Guzmán, V. V., et al. 2019, A\&A, 623, A124

Carr, J. S., \& Najita, J. R. 2008, Science, 319, 1504

Cazaux, S., \& Tielens, A. G. G. M. 2004, ApJ, 604, 222

Chapillon, E., Guilloteau, S., Dutrey, A., \& Piétu, V. 2008, A\&A, 488, 565

Chapillon, E., Dutrey, A., Guilloteau, S., et al. 2012, ApJ, 756, 58

Cleeves, L. I., Bergin, E. A., Qi, C., Adams, F. C., \& Öberg, K. I. 2015, ApJ, 799, 204

Collings, M. P., Anderson, M. A., Chen, R., et al. 2004, MNRAS, 354, 1133

Cuppen, H. M., \& Herbst, E. 2005, MNRAS, 361, 565

Dartois, E., Dutrey, A., \& Guilloteau, S. 2003, A\&A, 399, 773

de Gregorio-Monsalvo, I., Ménard, F., Dent, W., et al. 2013, A\&A, 557, A133

Di Folco, E., Péricaud, J., Dutrey, A., et al. 2020, A\&A, 635, A94

Dominik, C., Ceccarelli, C., Hollenbach, D., \& Kaufman, M. 2005, ApJ, 635, L85

Dong, R., Zhu, Z., \& Whitney, B. 2015, ApJ, 809, 93

Draine, B. T. 1978, ApJS, 36, 595

Draine, B. T., \& Lee, H. M. 1984, ApJ, 285, 89

Du, F., \& Bergin, E. A. 2014, ApJ, 792, 2

Duley, W. W. 1996, MNRAS, 279, 591

Dutrey, A., Guilloteau, S., \& Guelin, M. 1997, A\&A, 317, L55

Dutrey, A., Guilloteau, S., Piétu, V., et al. 2017, A\&A, 607, A130

Eistrup, C., Walsh, C., \& van Dishoeck, E. F. 2018, in IAU Symposium, 332, eds M. Cunningham, T. Millar, \& Y. Aikawa, 69

France, K., Schindhelm, E., Bergin, E. A., Roueff, E., \& Abgrall, H. 2014, ApJ, 784,127

Fromang, S., \& Nelson, R. P. 2009, A\&A, 496, 597

Garaud, P., \& Lin, D. N. C. 2004, ApJ, 608, 1050

Ge, J. X., He, J. H., \& Li, A. 2016, MNRAS, 460, L50

Goldman, N., Reed, E. J., Fried, L. E., William Kuo, I. F., \& Maiti, A. 2010, Nat. Chem., 2, 949

Gorti, U., Hollenbach, D., Najita, J., \& Pascucci, I. 2011, ApJ, 735, 90

Habart, E., Boulanger, F., Verstraete, L., Walmsley, C. M., \& Pineau des Forêts, G. 2004, A\&A, 414, 531

Habart, E., Abergel, A., Boulanger, F., et al. 2011, A\&A, 527, A122 
S. Gavino et al.: Impact of size-dependent grain temperature on chemistry in protoplanetary disks

Hasegawa, T. I., \& Herbst, E. 1993, MNRAS, 263, 589

Hasegawa, T. I., Herbst, E., \& Leung, C. M. 1992, ApJS, 82, 167

Heays, A. N., Bosman, A. D., \& van Dishoeck, E. F. 2017, A\&A, 602, A105

Heese, S., Wolf, S., Dutrey, A., \& Guilloteau, S. 2017, A\&A, 604, A5

Hersant, F., Wakelam, V., Dutrey, A., Guilloteau, S., \& Herbst, E. 2009, A\&A, 493, L49

Hollenbach, D., \& Gorti, U. 2009, ApJ, 703, 1203

Hudson, R. L., \& Gerakines, P. A. 2018, ApJ, 867, 138

Hughes, A. M., Duchêne, G., \& Matthews, B. C. 2018, ARA\&A, 56, 541

Iqbal, W., \& Wakelam, V. 2018, A\&A, 615, A20

Iqbal, W., Acharyya, K., \& Herbst, E. 2012, ApJ, 751, 58

Iqbal, W., Acharyya, K., \& Herbst, E. 2014, ApJ, 784, 139

Jura, M. 1975, ApJ, 197, 581

Katz, N., Furman, I., Biham, O., Pirronello, V., \& Vidali, G. 1999, ApJ, 522, 305

Kóspál, Á., Moór, A., Juhász, A., et al. 2013, ApJ, 776, 77

Laor, A., \& Draine, B. T. 1993, ApJ, 402, 441

Le Bourlot, J., Le Petit, F., Pinto, C., Roueff, E., \& Roy, F. 2012, A\&A, 541, A76

Lecar, M., Podolak, M., Sasselov, D., \& Chiang, E. 2006, ApJ, 640, 1115

Lee, H. H., Herbst, E., Pineau des Forets, G., Roueff, E., \& Le Bourlot, J. 1996, A\&A, 311, 690

Mie, G. 1908, Ann. Phys., 330, 377

Minissale, M., Moudens, A., Baouche, S., Chaabouni, H., \& Dulieu, F. 2016, MNRAS, 458, 2953

Mumma, M. J., \& Charnley, S. B. 2011, ARA\&A, 49, 471

Noble, J. A., Theule, P., Mispelaer, F., et al. 2012, A\&A, 543, A5

Ober, F., Wolf, S., Uribe, A. L., \& Klahr, H. H. 2015, A\&A, 579, A105

Öberg, K. I., Qi, C., Fogel, J. K. J., et al. 2011, ApJ, 734, 98

Öberg, K. I., Guzmán, V. V., Furuya, K., et al. 2015, Nature, 520, 198
Pauly, T., \& Garrod, R. T. 2016, ApJ, 817, 146

Péricaud, J., Di Folco, E., Dutrey, A., Guilloteau, S., \& Piétu, V. 2017, A\&A, 600, A62

Phuong, N. T., Chapillon, E., Majumdar, L., et al. 2018, A\&A, 616, L5

Podio, L., Kamp, I., Codella, C., et al. 2013, ApJ, 766, L5

Pontoppidan, K. M., Salyk, C., Blake, G. A., et al. 2010, ApJ, 720, 887 Reissl, S., Wolf, S., \& Brauer, R. 2016, A\&A, 593, A87

Riviere-Marichalar, P., Ménard, F., Thi, W. F., et al. 2012, A\&A, 538, A3

Ruaud, M., \& Gorti, U. 2019, ApJ, 885, 146

Ruaud, M., Wakelam, V., \& Hersant, F. 2016, MNRAS, 459, 3756

Semenov, D., \& Wiebe, D. 2011, ApJS, 196, 25

Sternberg, A., \& Dalgarno, A. 1995, ApJS, 99, 565

Thi, W. F., Hocuk, S., Kamp, I., et al. 2020, A\&A, 634, A42

van Dishoeck, E. F., \& Black, J. H. 1982, ApJ, 258, 533

Vidali, G., Roser, J. E., Manicó, G., \& Pirronello, V. 2004, J. Geophys. Res. (Planets), 109, E07S14

Vidali, G., Roser, J., Manicó, G., et al. 2005, J. Phys. Conf. Ser., 6, 36

Visser, R., van Dishoeck, E. F., \& Black, J. H. 2009, A\&A, 503, 323

Wakelam, V., Ruaud, M., Hersant, F., et al. 2016, A\&A, 594, A35

Wakelam, V., Loison, J. C., Mereau, R., \& Ruaud, M. 2017, Mol. Astrophys., 6, 22

Wakelam, V., Chapillon, E., Dutrey, A., et al. 2019, MNRAS, 484, 1563

Walsh, C., Millar, T. J., Nomura, H., et al. 2014, A\&A, 563, A33

Walsh, C., Loomis, R. A., Öberg, K. I., et al. 2016, ApJ, 823, L10

Williams, J. P., \& Best, W. M. J. 2014, ApJ, 788, 59

Woitke, P., Kamp, I., \& Thi, W. F. 2009, A\&A, 501, 383

Woitke, P., Kamp, I., Antonellini, S., et al. 2019, PASP, 131, 064301

Wolf, S. 2003, ApJ, 582, 859

Wolf, S., \& Voshchinnikov, N. V. 2004, Comput. Phys. Commun., 162, 113 


\section{Appendix A: Formal description of the model}

Unless otherwise specified, we use subscript $g$ for gas quantities and subscript $d$ for dust. Subscript 0 is used for reference values.

\section{Appendix A.1: Gas disk structure}

We describe the gas temperature and disk structure in this section. We neglect gas pressure and disk self-gravity, and thus assume that the disk is in Keplerian rotation around the host star as follows:

$v_{g}(r)=\sqrt{\frac{G M_{*}}{r}} \Longleftrightarrow \Omega_{g}(r)=\sqrt{\frac{G M_{*}}{r^{3}}}$,

where $r$ is the radius.

The gas surface density $\Sigma_{g}(r)$ also follows a power law

$\Sigma_{g}(r)=\Sigma_{g, 0}\left(\frac{r}{R_{0}}\right)^{-p}$

and, by integration, the gas surface density $\Sigma_{g, 0}$ at the reference radius $R_{0}$ for $p \neq 2$ is related to the gas disk mass by

$\Sigma_{g, 0}=\frac{M_{g} R_{0}^{-p}(2-p)}{2 \pi\left(R_{\text {out }}^{2-p}-R_{\text {in }}^{2-p}\right)}$,

where $R_{\text {in }}$ and $R_{\text {out }}$ are the inner and outer radii of the disk.

The gas temperature is not self-consistently derived from the dust temperature distribution but imposed by analytical laws. The kinetic temperature $T_{k}$ in the midplane is given by

$T_{k}(r, z=0)=T_{\text {mid, } 0}\left(\frac{r}{R_{0}}\right)^{-q}$.

Following Dartois et al. (2003), we allow for a warmer disk atmosphere using the following formulation of Williams \& Best (2014):

$T_{\mathrm{g}}(r, z)=T_{\text {mid }}(r)+\left(T_{\text {atm }}(r)-T_{\text {mid }}(r)\right) \sin \left(\frac{\pi z}{2 z_{\text {atm }}}\right)^{2 \sigma}$,

where $\sigma$ is the stiffness of the vertical temperature profile and $z_{\text {atm }}$ is the altitude at the upper boundary of our disk model (4 scale heights $H$ ), meaning that the temperature above $4 \mathrm{H}$ is constant. The atmosphere temperature is also given by a power law with the same exponent as that of the midplane temperature as follows:

$T_{\text {atm }}(r)=T_{\text {atm }, 0}\left(\frac{r}{R_{0}}\right)^{-q}$.

Isothermal case: If the gas temperature is vertically isothermal, we can derive the vertical density structure by assuming hydrostatic equilibrium at the midplane temperature. Consequently the gas vertical mass distribution follows a Gaussian profile

$\rho_{g}(r, z)=\rho_{g, \text { mid }}(r) \exp \left(-\frac{z^{2}}{2 H_{\mathrm{g}}(r)^{2}}\right)$,

where $\rho_{\mathrm{g}, \text { mid }}(\mathrm{r})$ is the density in the midplane, that is related to the surface density by

$\rho_{g, \text { mid }}(r)=\frac{\Sigma_{g}(r)}{\sqrt{2 \pi} H_{g}(r)}$.

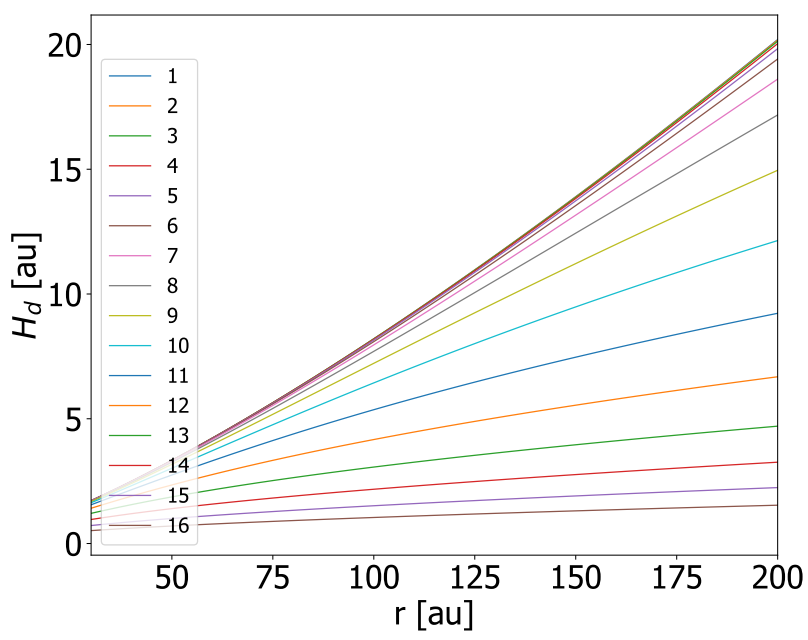

Fig. A.1: Scale heights of the grain distribution for the 16 different grain sizes as a function of the radius. The grain averaged size interval ranges from 0.007 to $651 \mu \mathrm{m}$. Small grains scale heights (size $<0.317 \mu \mathrm{m}$ ) practically follow the gas scale height. For larger grains the settling factor (Eq. A.21) becomes significant and the dust scale height decreases.

The scale height $H_{g}(r)$ is given by

$H_{g}(r)=\frac{C_{s}(r)}{\Omega_{g}(r)}$,

and using the definition of the temperature law from Eq.A.4

$H_{g, 0}=\frac{C_{s}\left(R_{0}\right)}{\Omega\left(R_{0}\right)}=\sqrt{\frac{k_{\mathrm{b}} T_{\mathrm{mid}, 0} R_{0}^{3}}{\mu_{\mathrm{m}} m_{H} G M_{*}}}$,

where $\mu_{\mathrm{m}}$ is the mean molecular weight, and $m_{\mathrm{H}}$ the hydrogen mass. The temperature and velocity being power laws, the scale height $H(r)$ also follows a power law

$H_{g}(r)=H_{g, 0}\left(\frac{r}{R_{0}}\right)^{\frac{3}{2}-\frac{q}{2}}$.

Vertical temperature gradient: With a vertical temperature gradient, the vertical gas profile deviates from a Gaussian profile. In Nautilus, we compute the gas density for each vertical cell $z_{i}$ at a given $r$ by solving out locally the equation as in Hersant et al. (2009),

$\ln \left(\rho_{g}\left(z_{i}\right)=\ln \left(\rho_{g}\left(z_{i}-1\right)\right)-\left(\Omega^{2} \frac{\mu m_{H}}{k_{B} T_{g}\left(z_{i}\right)}+\left(\ln \left(T_{g}\left(z_{i}\right)\right)-\ln \left(T_{g}\left(z_{i-i}\right)\right)\right)\right.\right.$.

\section{Appendix A.2: Dust distribution and properties}

\section{Appendix A.2.1: Dust material}

We assume that the grains are made of an homogeneous mixture of a population of silicate grains $(62.5 \%)$ and graphite grains $(37.5 \%)$. This composition is used for radiative transfer and chemical modeling. This leads to a material specific mass density for dust grains in all this study equal to $\rho_{\mathrm{m}}=2.5 \mathrm{~g} \cdot \mathrm{cm}^{-3}$. The grain temperatures are defined for each grain sizes using radiative transfer simulations as described in Section 2.2.2. 
S. Gavino et al.: Impact of size-dependent grain temperature on chemistry in protoplanetary disks

\section{Appendix A.2.2: Size distribution}

We consider a size dependent grain population. Overall, this grain population is derived assuming no radial drift, but accounting for dust settling as a function of height. The surface density of a grain population of size $a$ is thus given by

$\sigma_{d}(r, a)=\sigma_{d, 0}(a)\left(\frac{r}{R_{0}}\right)^{-p}$,

where $\sigma_{d, 0}(a)$ is the surface density of grains of size a at reference radius. Eq. A.13 implies that the total dust surface density is written as

$\Sigma_{d}(r)=\int_{a} \sigma_{d}(r, a) d a$,

which we associate with the overall (vertically integrated, equivalently disk averaged) dust-to-gas mass ratio $\zeta$ through $\Sigma_{d}(r)=$ $\zeta \Sigma_{g}(r)$. The mass fraction of grains of size $[a, a+d a]$ is given by

$x_{a} d a=\frac{m(a) d n(a)}{\int_{a_{\min }}^{a_{\max }} m(a) d n(a)}=\frac{m(a) d n(a)}{m_{d u s t}}$,

where the size distribution in number $d n(a)$ is assumed to follow a power law of exponent $d$,

$d n(a)=C a^{-d} d a$

where $C$ is a normalization constant. The quantity $C$ is related to the dust mass by simply integrating over the size as follows:

$m_{\text {dust }}=\int_{a_{\min }}^{a_{\max }} m(a) d n(a)=\int_{a_{\min }}^{a_{\max }} \frac{4 \pi}{3} \rho_{m} C a^{3-d} d a$,

where $\rho_{\mathrm{m}}$ is the specific mass density defined in the previous section. Using Eq.A.15 and the definition of $\zeta$ yields

$\sigma_{d}(r, a)=x_{a} \zeta \Sigma_{g}(r)=\frac{\zeta \Sigma_{g}(r) a^{3-d}}{\int_{a_{\min }}^{a_{\max }} a^{3-d} d a}$.

For radiative transfer and chemistry simulations the grain size distribution is discretized into several logarithmically distributed subranges with relative dust masses of the $i^{\text {th }}$ grain size interval as follows:

$\frac{m_{d, i}}{m_{d}}=\frac{\int_{a_{\min , i}}^{a_{\max , i}} \frac{4}{3} \pi \rho_{\mathrm{grain}} a^{3} n(a) \mathrm{d} a}{\int_{a_{\min }}^{a_{\max }} \frac{4}{3} \pi \rho_{\mathrm{grain}} a^{3} n(a) \mathrm{d} a}=\frac{a_{\mathrm{max}, i}^{4-d}-a_{\mathrm{min}, i}^{4-d}}{a_{\max }^{4-d}-a_{\min }^{4-d}}$.

The relative dust mass per bin and the relative area per bin (which also quantifies the number of chemical sites) are given in Table A.1.

\section{Appendix A.2.3: Dust settling}

To account for size-dependent dust settling, we assume that the vertical profile for a given grain size has a Gaussian shape

$\rho_{d}(r, z, a)=\frac{\sigma_{d}(r, a)}{\sqrt{2 \pi} H_{d}(r, a)} \exp \left(-\frac{z^{2}}{2 H_{d}(r, a)^{2}}\right)$.

Because dust grains do not react to pressure forces in the same way as gas, the dust scale height differs from that of the gas. Following Boehler et al. (2013), we define the settling factor, that
Table A.1: Overview of the grain size intervals

\begin{tabular}{lclc}
\hline Bin & Averaged size $(\mu \mathrm{m})$ & $\begin{array}{l}\text { Relative } \\
\text { dust mass }\end{array}$ & Relative area \\
\hline 1 & 0.007 & $0.10 \%$ & $30.5 \%$ \\
2 & 0.015 & $0.15 \%$ & $20.8 \%$ \\
3 & 0.032 & $0.22 \%$ & $14.2 \%$ \\
4 & 0.069 & $0.33 \%$ & $9.73 \%$ \\
5 & 0.15 & $0.48 \%$ & $6.67 \%$ \\
6 & 0.32 & $0.70 \%$ & $4.59 \%$ \\
7 & 0.68 & $1.03 \%$ & $3.19 \%$ \\
8 & 1.46 & $1.50 \%$ & $2.25 \%$ \\
9 & 3.13 & $2.20 \%$ & $1.64 \%$ \\
10 & 6.69 & $3.22 \%$ & $1.25 \%$ \\
11 & 14.4 & $4.72 \%$ & $1.03 \%$ \\
12 & 31.0 & $6.91 \%$ & $0.90 \%$ \\
13 & 66.0 & $10.1 \%$ & $0.84 \%$ \\
14 & 142 & $14.8 \%$ & $0.80 \%$ \\
15 & 304 & $21.7 \%$ & $0.78 \%$ \\
16 & 651 & $31.8 \%$ & $0.76 \%$
\end{tabular}

is the ratio of $H_{d}(r, a)$ to the gas scale height near the midplane $H_{g}(r)$, as

$s(a, r)=\frac{H_{d}(a, r)}{H_{g}(r)}$,

which can be related to the dust properties through the stopping time. The stopping time of a dust particle $\tau_{s}$ is the characteristic time for a dust particle initially at rest to reach the local gas velocity. We use the dimensionless stopping time $T_{s}(r, z)$ defined as the product of $\tau_{s}$ and the Keplerian angular momentum $\Omega(r)$, which is a way to compare the stopping time to the dynamical time in the disk. Garaud \& Lin (2004) showed that dust friction with gas in protoplanetary disks is well described by the Epstein regime, in which the dimensionless stopping time is given by

$T_{s}(r, z)=\tau_{s} \Omega_{g}(r)=\frac{a \rho_{m}}{\rho_{g}(r, z) C_{s}} \Omega(r)$.

The stopping time depends linearly on the grain size. Using Eq. A.9 we can write

$T_{s}(r, z)=\frac{a \rho_{m}}{\rho_{g}(r, z) H_{g}(r)}=\frac{\sqrt{2 \pi} a \rho_{m}}{\Sigma_{g}(r)} \exp \left(-\frac{z^{2}}{2 H_{g}(r)^{2}}\right)$.

The settling factor is given by the approximation of Dong et al. (2015), that is,

$s(a, r)=\frac{H_{d}(a, r)}{H_{g}(r)}=\frac{1}{\sqrt{1+T_{s, \text { mid }} \frac{S_{c}}{\alpha}}}$,

where $\alpha$ is the $\alpha$ viscosity coefficient,

$T_{s, \text { mid }}=\frac{\sqrt{2 \pi} a \rho_{m}}{\Sigma_{g}(r)}$

is the dimensionless stopping time in the midplane, and $S_{c}$ is the Schmidt number. The Schmidt number is a dimensionless number defined by the ratio of the turbulent viscosity $v_{t}$ to the turbulent diffusion $D_{t}$ as follows:

$S_{c}=\frac{v_{t}}{D_{t}}$ 


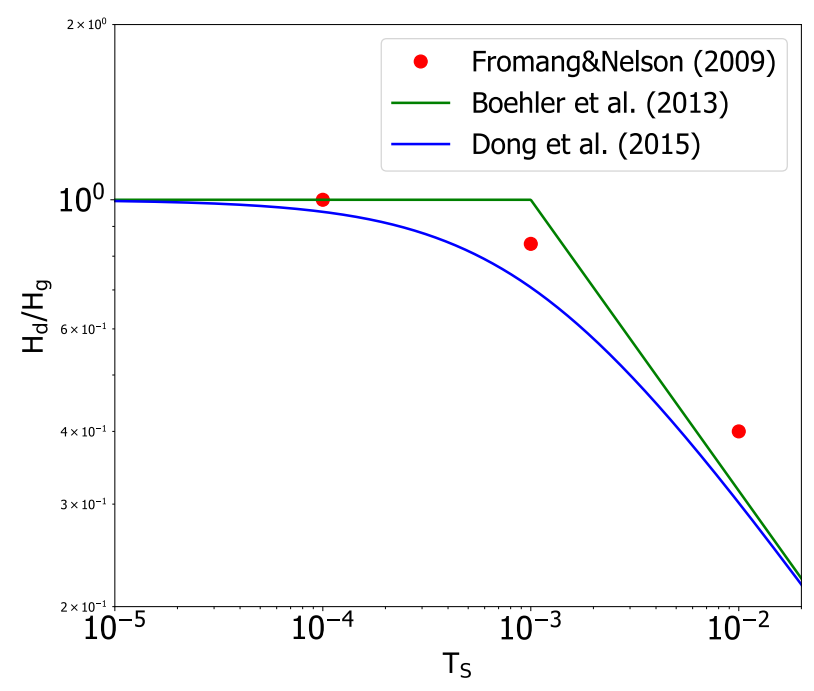

Fig. A.2: Settling factor as a function of the dimensionless stopping time at $R_{0}$. In green the model given by Boehler et al. (2013), the red dots show the simulation values of Fromang \& Nelson (2009), and the blue curve indicates the model of Dong et al. (2015) used in this work.

Table A.2: Total dust cross sections for each size interval in the midplane for three radii of the multi-grain model.

\begin{tabular}{lccc}
\hline & \multicolumn{3}{c}{ Total cross-section $\left(\mathrm{cm}^{-1}\right)$} \\
\cline { 2 - 4 } Size $(\mu \mathrm{m})$ & $50 \mathrm{au}$ & $100 \mathrm{au}$ & $200 \mathrm{au}$ \\
\hline 0.007 & $3.39(-14)$ & $4.86(-15)$ & $6.99(-16)$ \\
0.015 & $2.31(-14)$ & $3.32(-15)$ & $4.78(-16)$ \\
0.032 & $1.58(-14)$ & $2.27(-15)$ & $3.27(-16)$ \\
0.069 & $1.08(-14)$ & $1.55(-15)$ & $2.24(-16)$ \\
0.15 & $7.38(-15)$ & $1.06(-15)$ & $1.55(-16)$ \\
0.32 & $5.05(-15)$ & $7.32(-16)$ & $1.08(-16)$ \\
0.68 & $3.47(-15)$ & $5.08(-16)$ & $7.69(-17)$ \\
1.46 & $2.40(-15)$ & $3.59(-16)$ & $5.69(-17)$ \\
3.13 & $1.68(-15)$ & $2.61(-16)$ & $4.46(-17)$ \\
6.69 & $1.21(-15)$ & $2.00(-16)$ & $3.75(-17)$ \\
14.4 & $9.06(-16)$ & $1.64(-16)$ & $3.37(-17)$ \\
31.0 & $7.24(-16)$ & $1.44(-16)$ & $3.17(-17)$ \\
66.0 & $6.21(-16)$ & $1.34(-16)$ & $3.06(-17)$ \\
142 & $5.65(-16)$ & $1.28(-16)$ & $2.98(-17)$ \\
304 & $5.37(-16)$ & $1.25(-16)$ & $2.90(-17)$ \\
651 & $5.21(-16)$ & $1.22(-16)$ & $2.76(-17)$ \\
\hline
\end{tabular}

Figure A.2 shows the settling factor at the reference radius as defined in Dong et al. (2015) compared to the asymptotic behavior used by Boehler et al. (2013) and the values from the numerical simulations of Fromang \& Nelson (2009).

Table A. 2 provides the total dust cross section at 50, 100, and $200 \mathrm{au}$ from the star, defined by the term $n_{d}(r, z=0, a) \pi a^{2}$.

\section{Appendix B: Self and mutual shielding}

The local UV flux in the disk is not a simple sum of the attenuated stellar and interstellar fields because of scattering by dust grains. Scattering can be significant near the disk midplane, where the optical depths are large. However, molecular shielding
Table B.1: Adopted elemental initial abundances relative to $\mathrm{H}$.

\begin{tabular}{ccc}
\hline Element & Abundance (relative to H) & Mass (amu) \\
\hline $\mathrm{He}$ & $9.0(-2)$ & 4.00 \\
$\mathrm{C}$ & $1.7(-4)$ & 12.00 \\
$\mathrm{~N}$ & $6.2(-5)$ & 14.00 \\
$\mathrm{O}$ & $2.4(-4)$ & 16.00 \\
$\mathrm{Si}$ & $8.0(-9)$ & 28.00 \\
$\mathrm{~S}$ & $8.0(-8)$ & 32.00 \\
$\mathrm{Fe}$ & $3.0(-9)$ & 56.00 \\
$\mathrm{Na}$ & $2.0(-9)$ & 23.00 \\
$\mathrm{Mg}$ & $7.0(-9)$ & 24.00 \\
$\mathrm{Cl}$ & $1.0(-9)$ & 35.00 \\
$\mathrm{P}$ & $2.0(-10)$ & 31.00 \\
$\mathrm{~F}$ & $6.7(-9)$ & 19.00
\end{tabular}

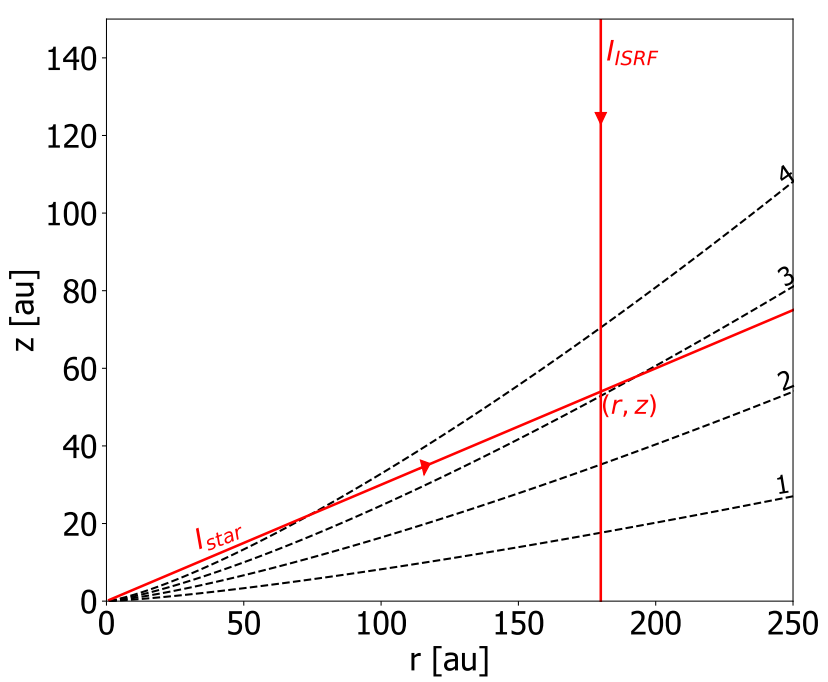

Fig. B.1: Elevation above the midplane as a function of the radius. The dotted black lines represent the scale heights of the gas $(1 \mathrm{H}, 2 \mathrm{H}, 3 \mathrm{H}$, and $4 \mathrm{H})$. The red lines indicate radiation emitted from the central star and from the ISRF. The coordinates $(r, z)$ represent a point at which both fields cross.

is most important only in the upper layers, when dust optical depth remains low while molecular optical depth can be significant.

Thus, at first order, to evaluate the impact of self-shielding, we neglect scattering in our analysis by writing in a simple form the local flux $I$ in the cell of coordinates $(r, z)$ as follows:

$I(\lambda, r, z) \approx I_{I S R F} e^{-\tau^{V}}+I_{*}(r, z) e^{-\tau^{R}}$,

where $I_{I S R F}$ is the incident flux from the $\operatorname{ISRF}_{I_{*}}(r, z)$ is the spatially diluted, unattenuated, incident flux from the central star, $\tau^{V}$ is the vertical opacity generated by the disk matter above the local point $(r, z)$, and $\tau^{R}$ is the radial opacity generated by the matter between the central star and the local point. As the opacities are a contribution of both dust and gas, we write

$$
\begin{aligned}
& \tau^{V}=\tau_{m}^{V}+\tau_{d}^{V} \\
& \tau^{R}=\tau_{m}^{R}+\tau_{d}^{R}
\end{aligned}
$$


where $m$ stands for molecules and $d$ for dust. So the local expression becomes

$I(\lambda, r, z) \approx I_{I S R F} e^{-\left(\tau_{d}^{V}+\tau_{m}^{V}\right)}+I_{*} e^{-\left(\tau_{d}^{R}+\tau_{m}^{R}\right)}$

The local flux weighted by the dust only $I_{d}$ can also be approximated by

$I_{d}(\lambda, r, z) \approx I_{I S R F} e^{-\tau_{d}^{V}}+I_{*} e^{-\tau_{d}^{R}}$.

The POLARIS code treats cscattering by dust consistently and this simple expression was checked to be a reasonable approximation in the upper layers where molecular shielding is important.

At a given wavelength, the molecular opacity is defined as follows:

$\tau_{m}(\lambda, r, z)=\sum_{X} \int_{l=f(r, z)} n(X, r, z) \sigma(X, \lambda) d l$,

where $X$ includes all molecules for which we have cross sections (including $\left.\mathrm{H}_{2} \mathrm{O}\right), n(X, r, z)$ is the number density $\left[\mathrm{cm}^{-3}\right]$ of species $X$ at coordinates $(r, z)$, and $\sigma(X, \lambda)$ is the sum of the absorption, dissociation, and ionization cross sections of species $X$ at wavelength $\lambda$. Depending on whether the radiation is from the interstellar medium or from the central star, the line $l$ over which we integrate is either the altitude $z$ above the cell of coordinates $(r, z)$ or the radial distance $\sqrt{r^{2}+z^{2}}$ between the central star and the considered cell, respectively.

Figure B.2 shows the vertical opacities generated by the dust (dotted lines) and the molecules (solid lines). The opacities are given for different altitudes at $100 \mathrm{au} . \mathrm{H}_{2}, \mathrm{CO}$ and $\mathrm{N}_{2}$ are the main contributors to the line opacity.

As shown in Figs. B.2, at all wavelengths where $\mathrm{H}_{2}$ contributes to the attenuation $(\lambda \lesssim 115 \mathrm{~nm})$, its opacity is in all parts of the disk always substantially larger than the dust opacity.

While calculating the vertical molecular opacity $\tau_{m}^{V}$ is trivial, characterizing the radial molecular opacity $\tau_{m}^{R}$ is more difficult as it couples different radii and thus their chemical evolution together. Given the significant computing time involved in the multi-grain model, we show below that we can reasonably simplify the problem through a simple approximation.

Self or mutual shielding is only relevant for high molecular opacities, because frequencies at which molecular shielding occurs cover a limited fraction of the UV domain. In regard to Figure B.1, we introduce the ratio of the radial to vertical molecular optical depths as follows:

$R V=\frac{\tau_{m}^{R}}{\tau_{m}^{V}}$

and Eq. B.3 can be rewritten as

$I(\lambda, r, z) \approx e^{-\tau_{m}^{V}}\left(I_{I S R F} e^{-\tau_{d}^{V}}+I_{*} e^{-\tau_{d}^{R}} e^{-\tau_{m}^{V}(R V-1)}\right)$.

Strictly speaking, $R V$ is position and frequency dependent, since the radial and vertical distribution of molecules differ.

The geometry shown in Figure B.1 indicates that $R V$ should be $\geq 1$ and we approximate Eq. B.7 by

$$
I(\lambda, r, z) \approx e^{-\tau_{m}^{V}}\left(I_{I S R F} e^{-\tau_{d}^{V}}+I_{*} e^{-\tau_{d}^{R}}\right) \approx e^{-\tau_{m}^{V}} I_{d}(\lambda, r, z) .
$$

This approximation may underestimate the molecular shielding when $\tau_{m}^{V}$ is $\lesssim 1$, while $\tau_{m}^{R}$ is substantially larger than 1 ,

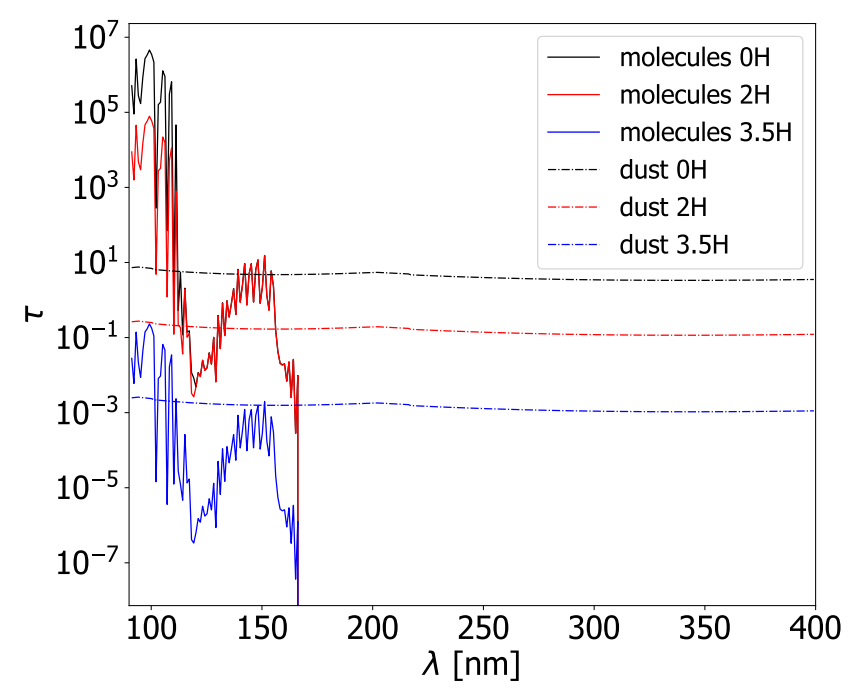

Fig. B.2: Vertical optical depth as a function of wavelengths given at different scale heights $H_{\mathrm{g}}(0 \mathrm{H}, 2 \mathrm{H}, 4 \mathrm{H})$ of a disk at $100 \mathrm{au}$ away from the star. The solid lines represent molecular opacities; the dotted lines indicate dust opacities.

thus impacting the details of the $\mathrm{H}_{2}$ formation layer and more generally the physico-chemistry in the upper disk atmosphere. Studying the disk upper layer is out of the scope of our paper (and would require the use of a PDR code). In fact, our approximation becomes reasonable as soon as dust attenuation is significant; this situation happens at $z / H<3.5-3.8$ in our dust disk model, which has a relatively high dust mass, and does not affect our results on the molecular layer and below.

In summary, self and mutual shielding are considered throughout the disk, by applying the attenuation by the vertical opacity due to molecules to the radiation field computed with dust only by the POLARIS code as follows:

$I(\lambda, r, z)=e^{-\tau_{m}^{V}} I_{d}(\lambda, r, z)$

where $I_{d}(\lambda, r, z)$ explicitly includes the impact of dust scattering that is handled by POLARIS.

It is important to mention that compared to empirical, precomputed factors as a function of visual extinction and $\mathrm{H}_{2}$ column densities (or also $\mathrm{CO}$ and $\mathrm{N}_{2}$ for self-shielding), this approach has the advantage of consistently taking the dust extinction into account for the specific dust properties and distribution.

Consequently, we use Eq. B.9 to compute the photorates and the vertical molecular opacities are computed using Eq. B.5 in all our simulations. Figure B. 3 gives the flux as a function of the wavelength in the midplane and in the upper atmosphere $(4 \mathrm{H})$ at 100 au for the disk whose parameters are given in Table 1. It shows a comparison between the flux as given by the radiative transfer simulation (dotted lines) and the flux as given by Eq. 2, which takes into account the molecular contribution to the opacity (solid line). The attenuation by spectral lines, especially those of $\mathrm{H}_{2}$, is clearly visible. The contribution of the molecules to the opacity occurs in a limited range of the UV domain. Although our chemical code (Section 2.3) computes other molecular species that contribute to the UV opacity, their abundance relative to $\mathrm{H}_{2}$ remains small and limit their contribution. 


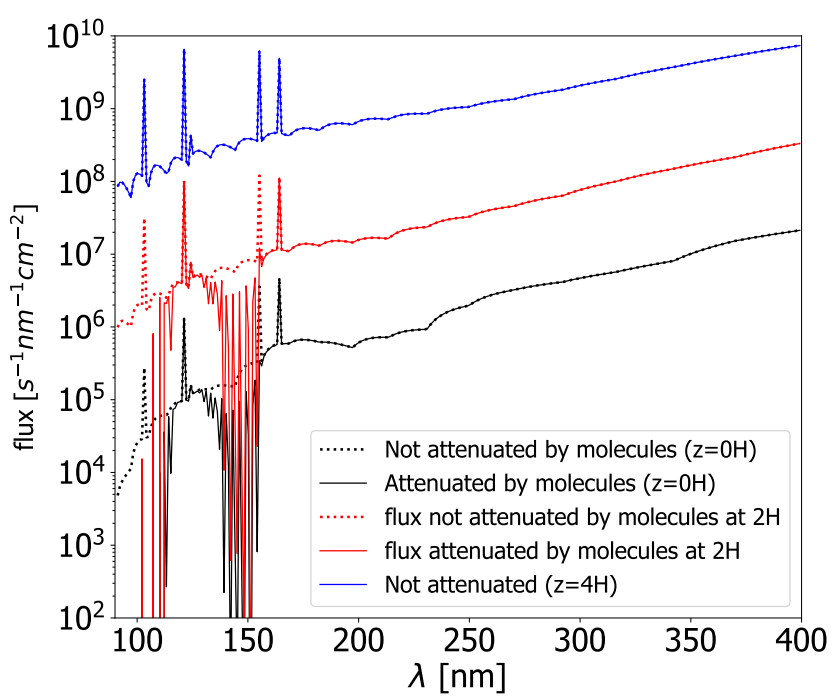

Fig. B.3: Flux as a function of the wavelength in the midplane (black lines), at $2 H$ (red lines), and at $4 H$ (blue lines) at 100 au. The unattenuated UV spectrum is derived from France et al. (2014) with spectral resampling preserving the equivalent widths of the lines.

\section{Appendix C: HUV cases}

In Figs.C.1 and C. 2 we present the vertical profiles at $100 \mathrm{au}$ of the fractional abundances and number densities of gas-phase $\mathrm{H}$, $\mathrm{H}_{2}, \mathrm{CO}, \mathrm{CS}$, and $\mathrm{CN}$ in HUV single-grain models and multigrain models, respectively.

\section{Appendix C.1: Intermediate models}

The intermediate models have physical conditions identical to that of the multi-grain models, but the dust grain distribution is represented using (locally) a single-grain size, determined so that its effective area and mass are identical to those of dust grains at the same location in multi-grain models. The temperature of this equivalent grain is the area-weighted temperature $T_{a}$. These models thus exhibit dust settling as multi-grain models. They also have a larger UV penetration than in single-models, similar, although this value is not identical to the UV penetration in multi-grain models.

For simplicity, we only compute the chemistry for the HUV flux at 50, 100, and 200 au. The results are presented in Figures. C.3, C.4, and have been added in Figures 10, 12, and 15 for discussion. 


\section{Appendix D: LUV cases}

In Figure D.1 we present the vertical cumulative column density of $\mathrm{H}_{2}, \mathrm{CO}, \mathrm{CS}$, and $\mathrm{CN}$ at 100 au from the LUV models. The profiles are very similar to that of the HUV models below $z \sim 2 H$ (see Fig. 11). We note a few noticeable differences. The model M-LUV-LH produces a larger column density of CS than M-HUV-LH. As for CN, it is clear that M-HUV-B14 produces a larger column density than M-HUV-LH. In the case of LUV multi-grain models, the difference is far less pronounced and M-LUV-LH produces a slightly larger column density than MLUV-B14. These different results are mostly due to the lower UV penetration in the case of the LUV models than in the HUV models.

In Figure D.2 we present maps of number density for $\mathrm{H}_{2}$, $\mathrm{CO}, \mathrm{CS}$, and $\mathrm{CN}$ in the case of LUV single-grain models. Again, the similarity with HUV models is clear. The profiles around the midplane are identical between LUV and HUV. The impact of the different flux on the upper layers is visible. The peak of $\mathrm{CO}$, $\mathrm{CS}$, and $\mathrm{CN}$ are located at higher altitudes and are globally wider in LUV models. We note one major difference for $\mathrm{CN}$ between LUV and HUV models. HUV-LH- $T_{\mathrm{a}}$ exhibits a clear spike of $\mathrm{CN}$ density around $2 H$ while, conversely, LUV-LH- $T_{\text {a }}$ exhibits a drop at the same location. Figs. D. 3 and D.4 show the vertical profiles at 100 au of the fractional abundances and number densities of gas-phase $\mathrm{H}, \mathrm{H}_{2}, \mathrm{CO}, \mathrm{CS}$, and $\mathrm{CN}$ in LUV single-grain models and multi-grain models, respectively. 

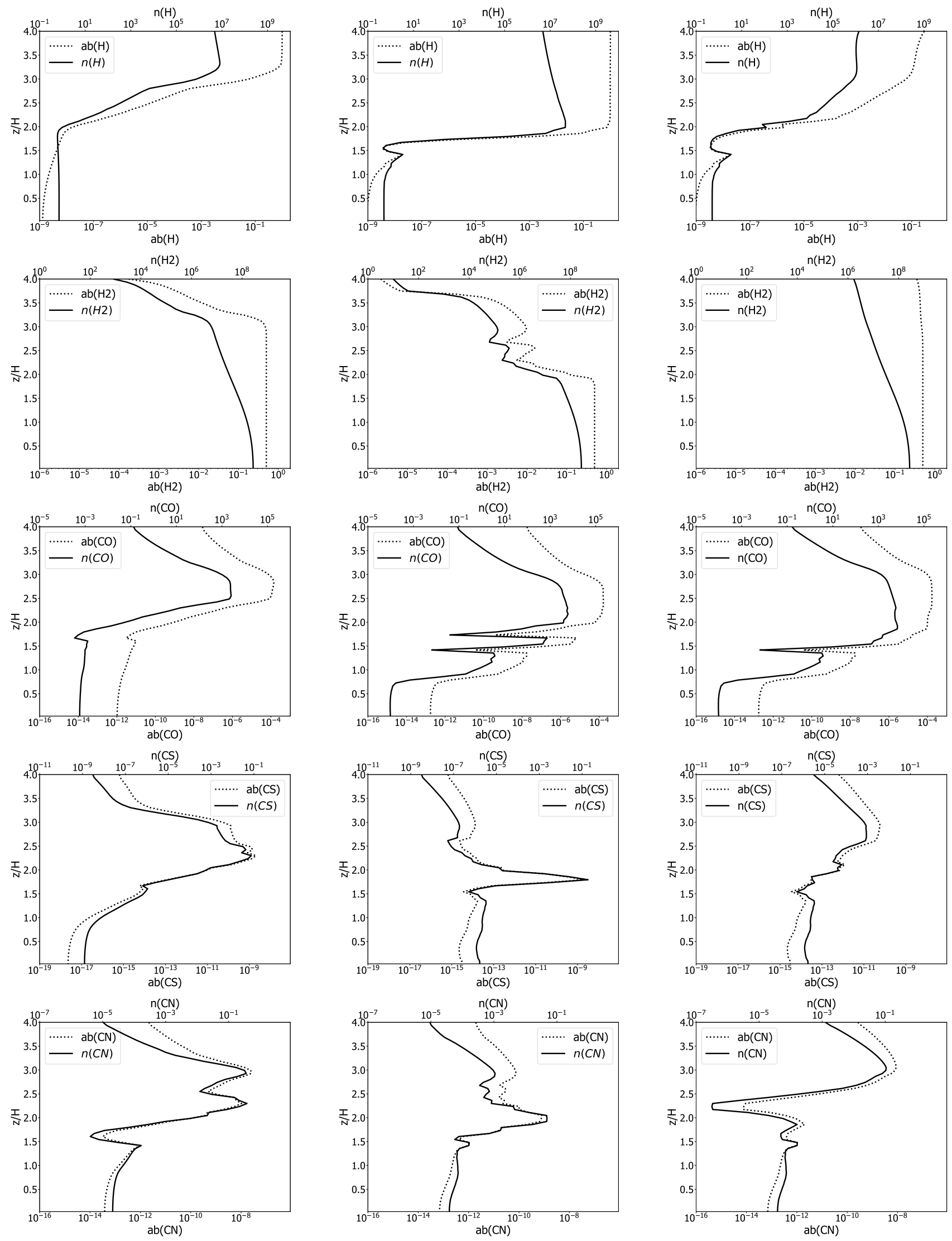

(a) HUV-LH-T $\mathrm{g}$

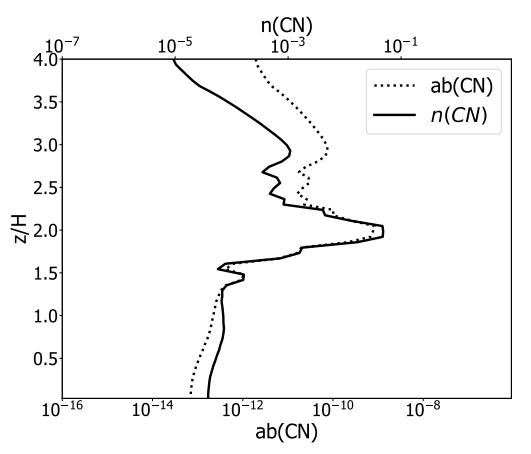

(b) HUV-LH-T

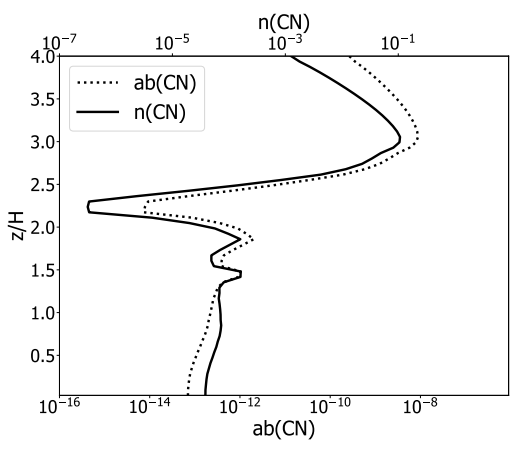

(c) HUV-B14-T

Fig. C.1: Vertical profiles of $\mathrm{H}, \mathrm{H}_{2}, \mathrm{CO}, \mathrm{CS}$, and $\mathrm{CN}$ at 100 au from the star of the HUV single-grain models. The dotted line is the abundance relative to $\mathrm{H}$ and the solid line is the density $\left[\mathrm{cm}^{-3}\right]$. 
S. Gavino et al.: Impact of size-dependent grain temperature on chemistry in protoplanetary disks
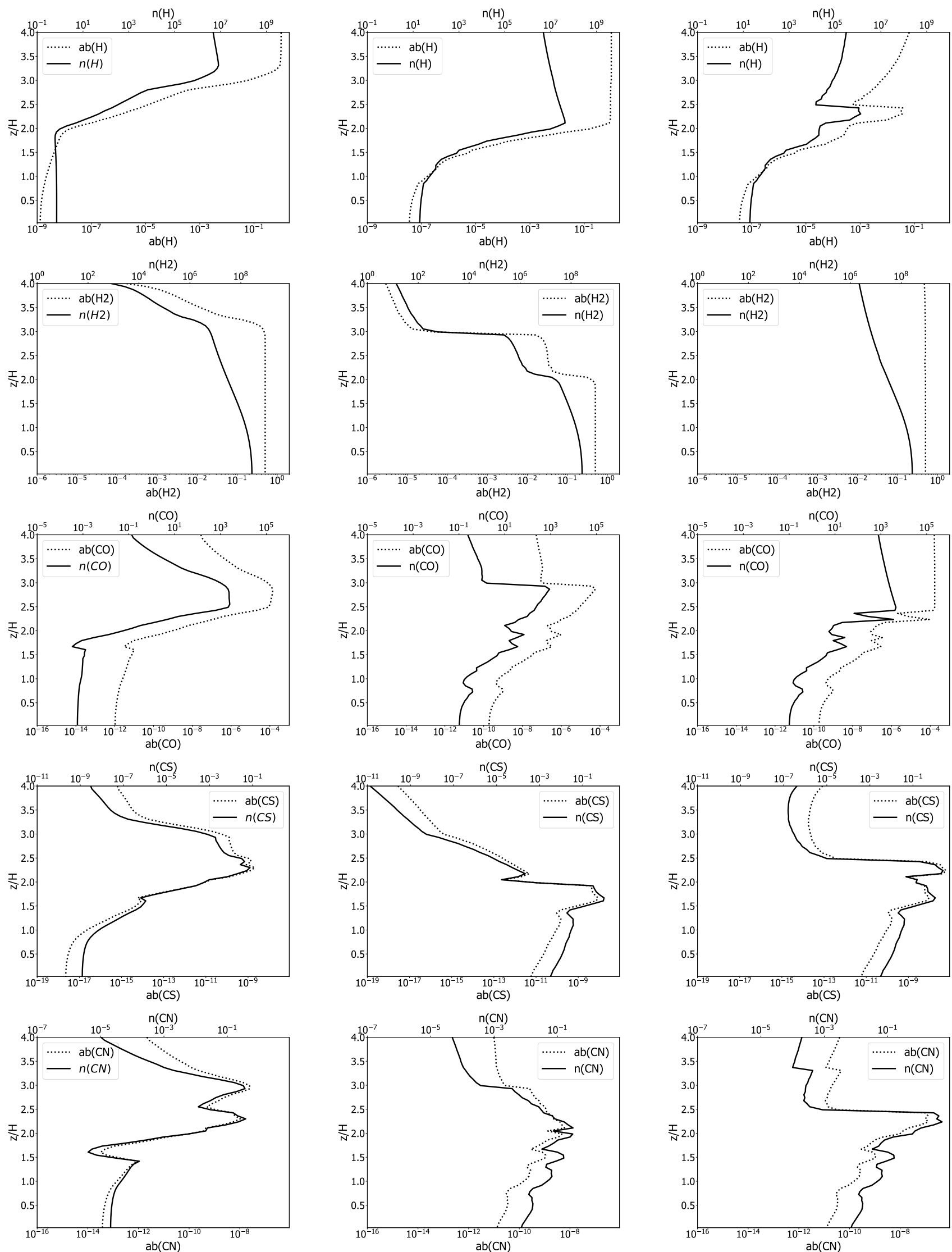

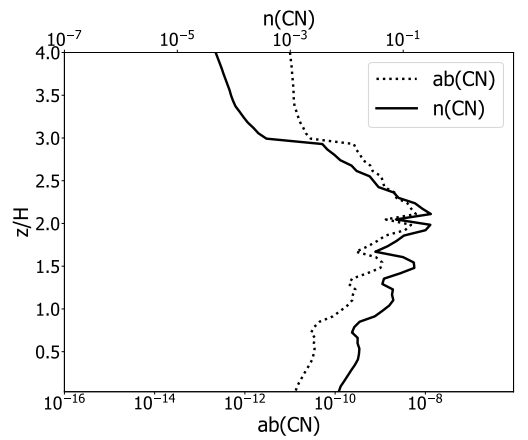

(b) M-HUV-LH

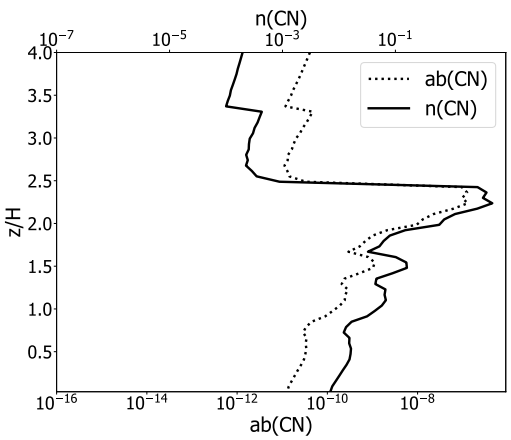

(c) M-HUV-B14

Fig. C.2: Vertical profiles of $\mathrm{H}, \mathrm{H}_{2}, \mathrm{CO}, \mathrm{CS}$, and $\mathrm{CN}$ at 100 au from the star of the $\mathrm{HUV}$ single-grain model HUV-LH- $T_{\mathrm{g}}$ on the left column, and multi-grain models on middle and right columns. The dotted line indicates the abundance relative to $\mathrm{H}$ and the solid line indicates the density $\left[\mathrm{cm}^{-3}\right]$. 

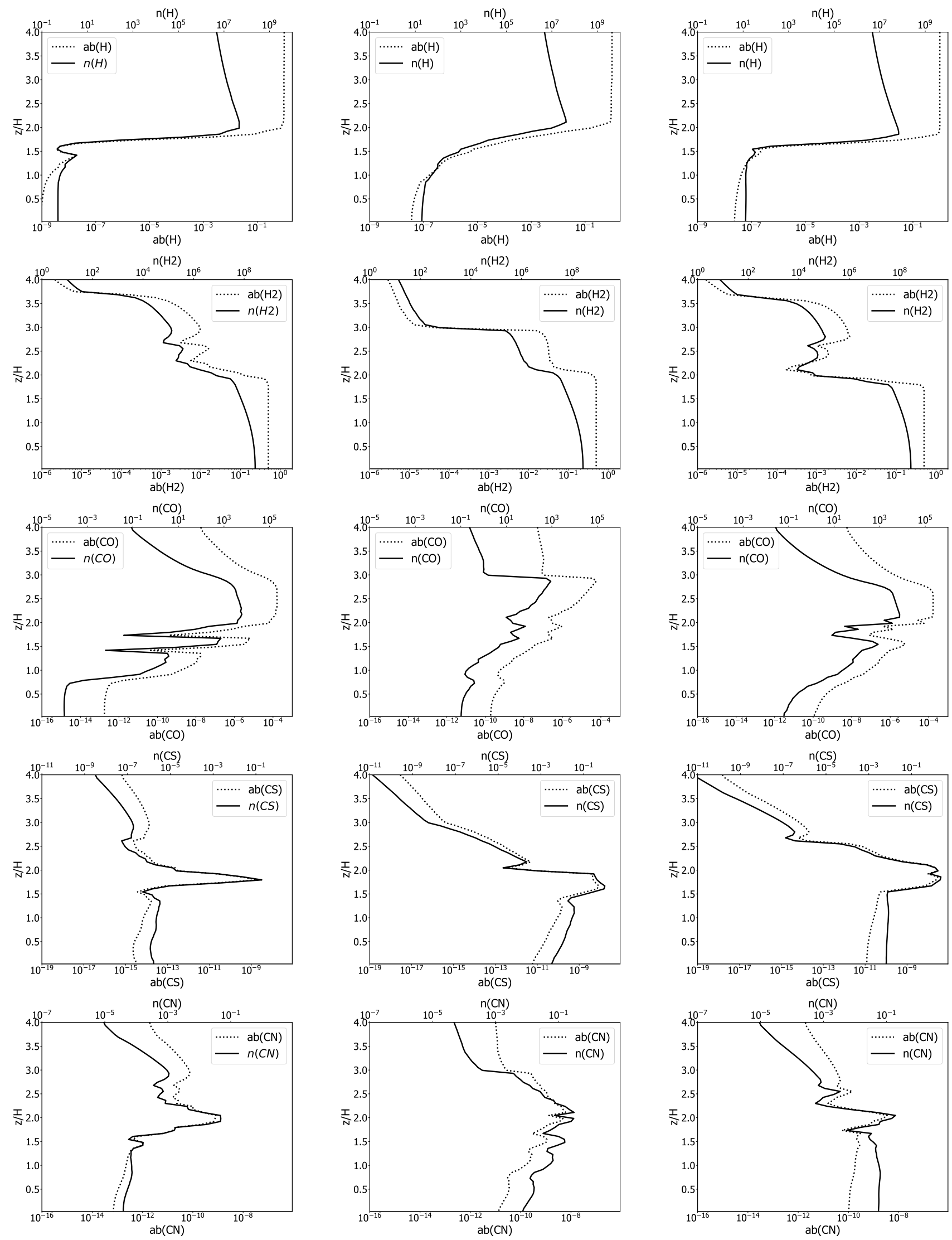

(a) HUV-LH-T

(b) M-HUV-LH

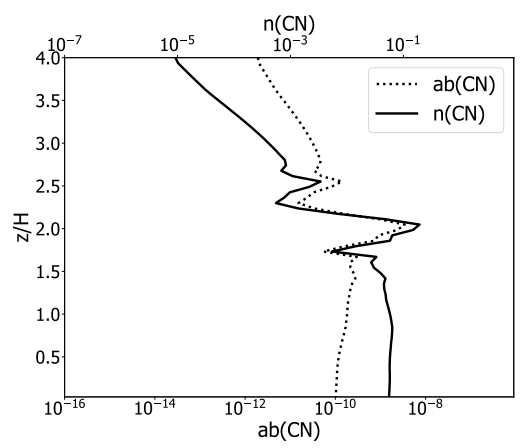

(c) Set-HUV-LH-Ta

Fig. C.3: Vertical profiles of $\mathrm{H}, \mathrm{H}_{2}, \mathrm{CO}, \mathrm{CS}$, and $\mathrm{CN}$ at 100 au from the star of the single-grain model HUV-LH- $\mathrm{T}_{\mathrm{a}}$ (left), the multigrain model M-HUV-LH (middle), and the intermediate model Set-HUV-LH- $\mathrm{T}_{\mathrm{a}}$ (right). The dotted line represents the abundance relative to $\mathrm{H}$ and the solid line indicates the density $\left[\mathrm{cm}^{-3}\right]$. 
S. Gavino et al.: Impact of size-dependent grain temperature on chemistry in protoplanetary disks
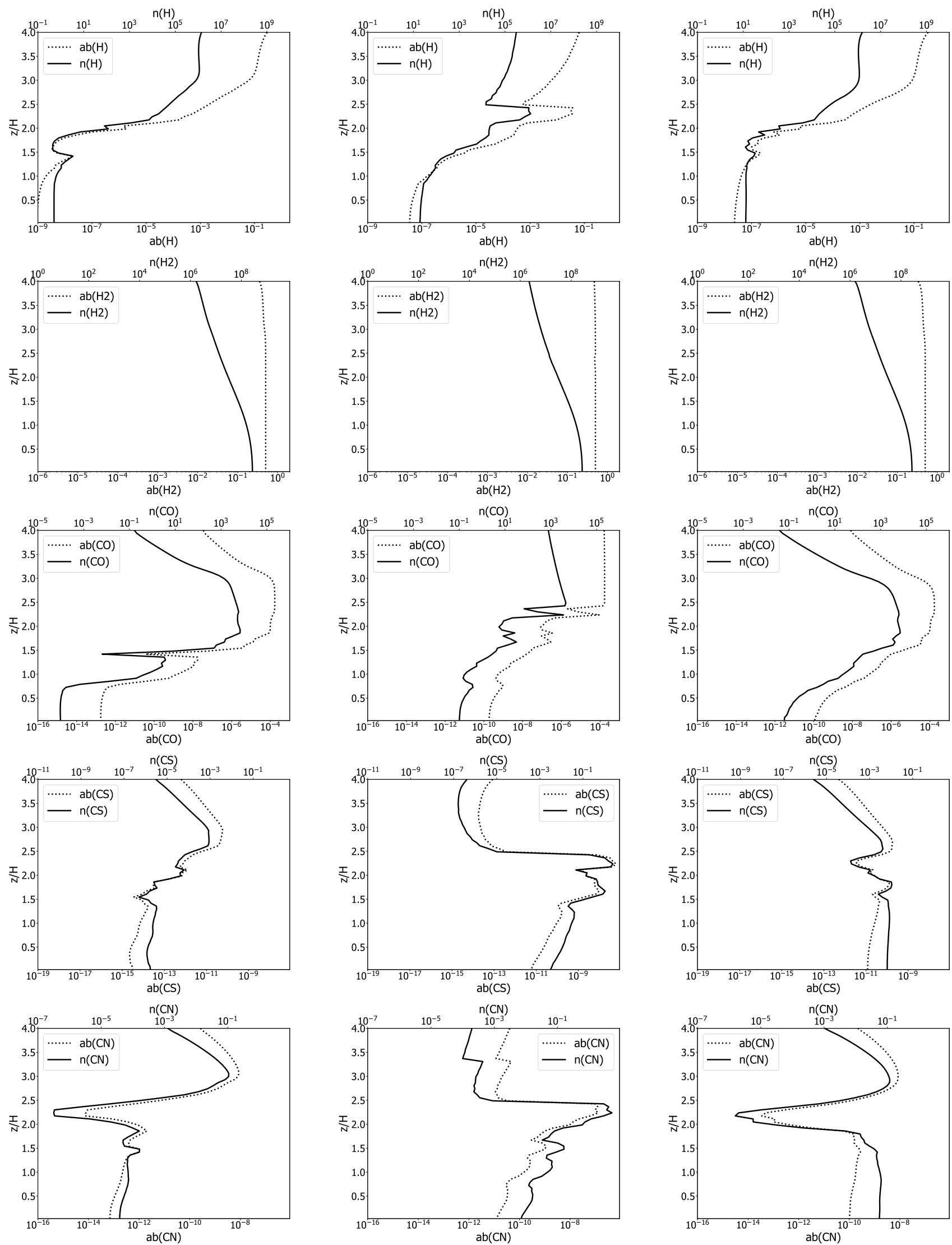

(a) HUV-B14-T

(b) M-HUV-B14

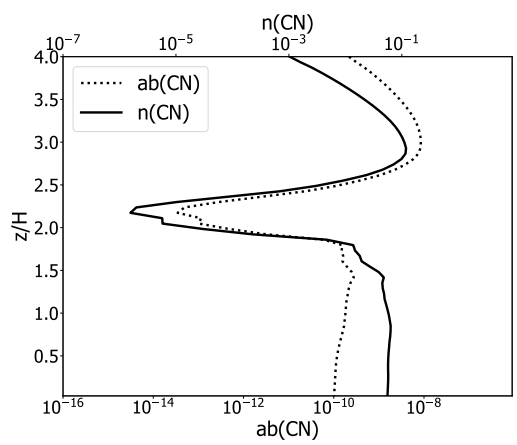

(c) Set-HUV-B14-Ta

Fig. C.4: Vertical profiles of $\mathrm{H}, \mathrm{H}_{2}, \mathrm{CO}, \mathrm{CS}$, and $\mathrm{CN}$ at 100 au from the star of the single-grain model HUV-B14- $\mathrm{T}_{\mathrm{a}}$ (left), the multigrain model M-HUV-B14 (middle), and the intermediate model Set-HUV-B $14-\mathrm{T}_{\mathrm{a}}$ (right). The dotted line represents the abundance relative to $\mathrm{H}$ and the solid line represents the density $\left[\mathrm{cm}^{-3}\right]$. 
Table D.1: LUV cases: Column densities of different species at 100 au for all models.

\begin{tabular}{cccccc}
\hline & $\mathrm{H}$ & $\mathrm{H}_{2}$ & $\mathrm{CO}$ & $\mathrm{CS}$ & $\mathrm{CN}$ \\
\hline \hline LUV-LH-Tg & $9.70 .10^{20}$ & $8.22 .10^{22}$ & $4.11 .10^{17}$ & $2.34 .10^{12}$ & $1.01 .10^{13}$ \\
\hline LUV-HL-Ta & $7.89 .10^{21}$ & $7.87 .10^{22}$ & $1.21 .10^{18}$ & $3.75 .10^{12}$ & $1.87 .10^{12}$ \\
\hline LUV-B14-Ta & $9.68 .10^{19}$ & $8.26 .10^{22}$ & $1.47 .10^{18}$ & $2.96 .10^{11}$ & $2.25 .10^{13}$ \\
\hline M-LUV-LH & $6.70 .10^{21}$ & $7.93 .10^{22}$ & $1.79 .10^{17}$ & $3.55 .10^{13}$ & $5.87 .10^{13}$ \\
\hline M-LUV-B14 & $1.34 .10^{19}$ & $8.27 .10^{22}$ & $1.15 .10^{18}$ & $1.33 .10^{13}$ & $4.67 .10^{13}$
\end{tabular}

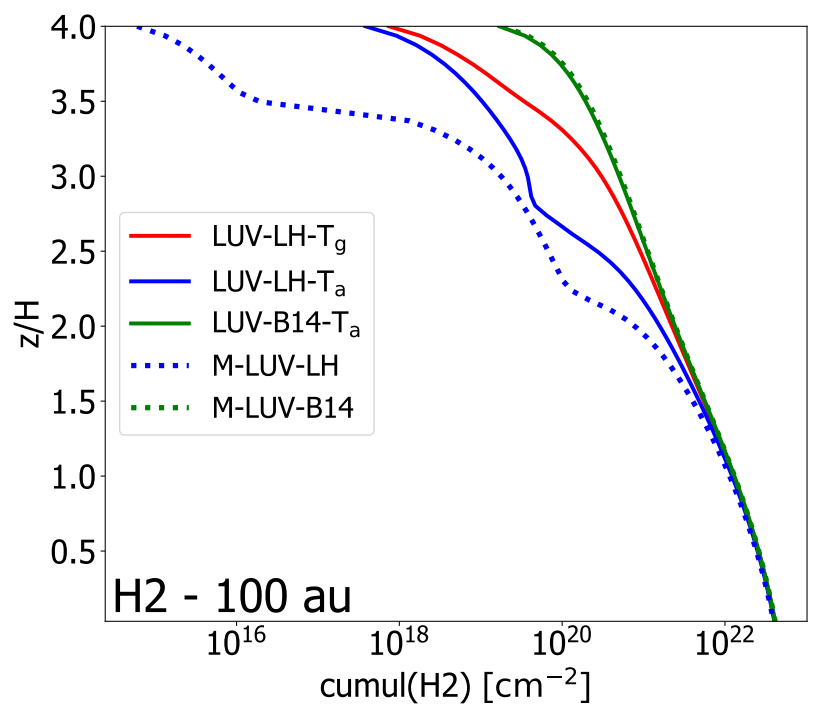

(a) $\mathrm{H} 2$

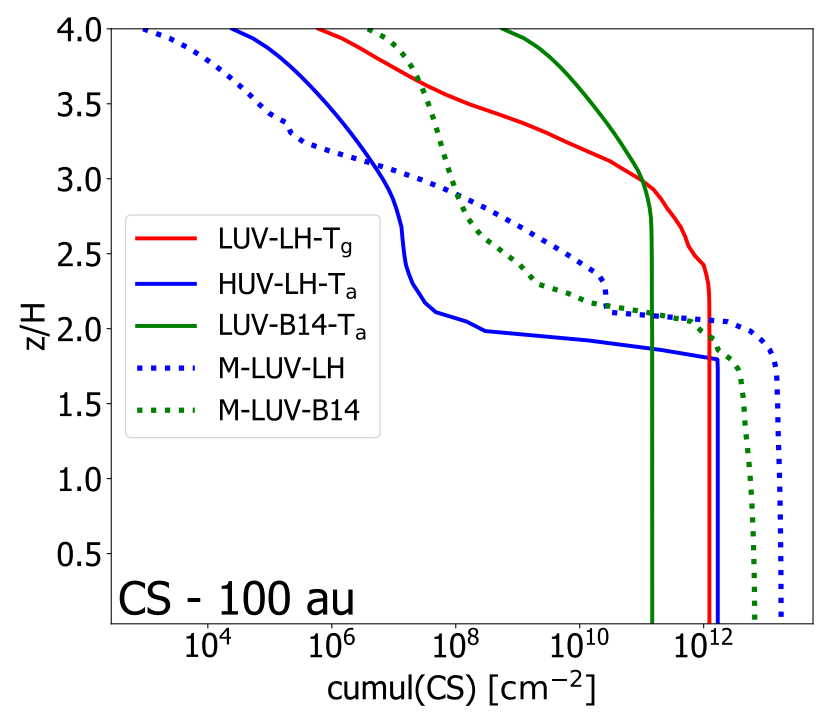

(c) CS

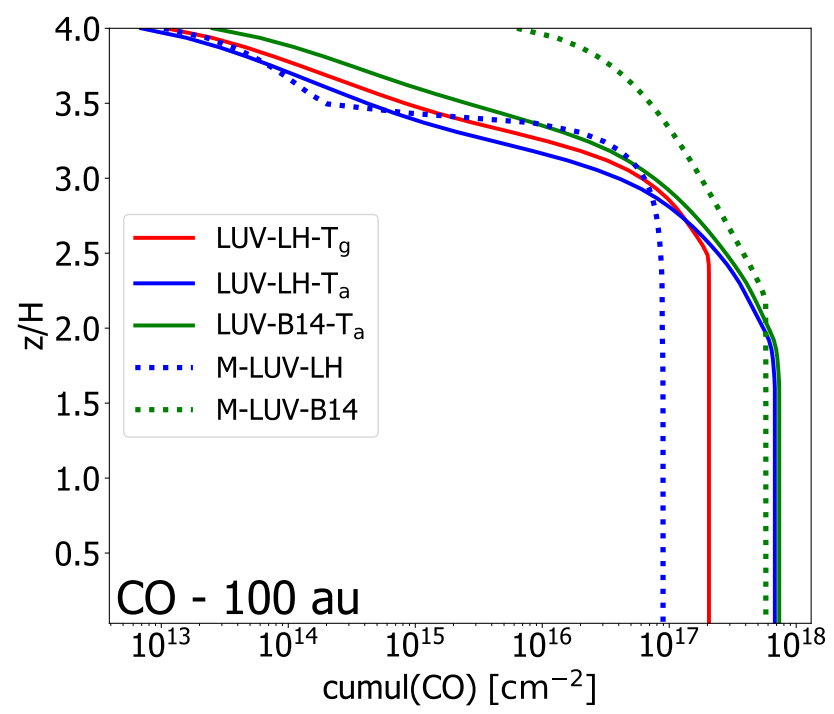

(b) $\mathrm{CO}$

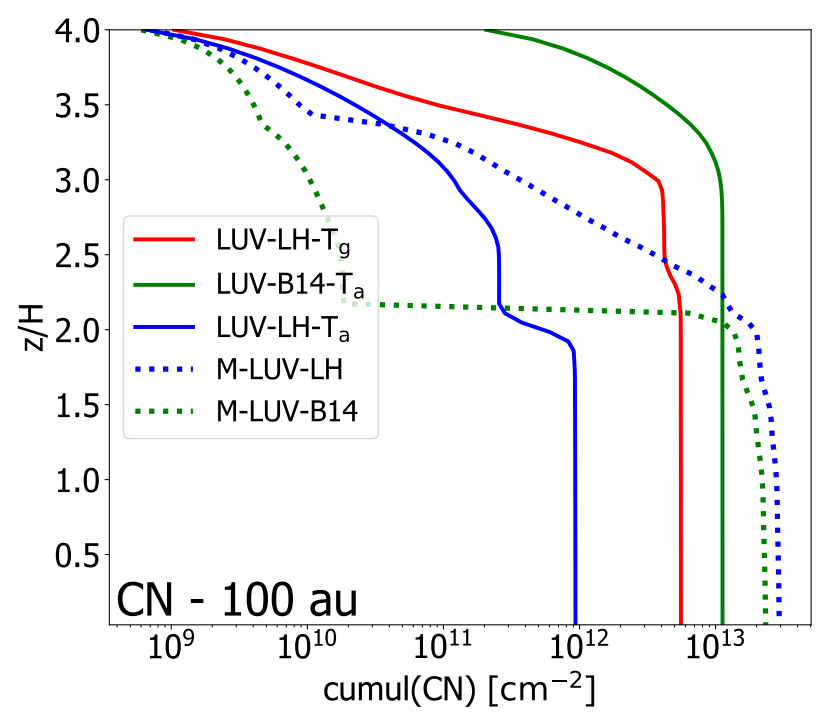

(d) $\mathrm{CN}$

Fig. D.1. Vertical cumulative surface density $\left[\mathrm{cm}^{-2}\right]$ of $\mathrm{H}_{2}, \mathrm{CO}, \mathrm{CS}$, and $\mathrm{CN}$ at 100 au from the star of the LUV models. 
S. Gavino et al.: Impact of size-dependent grain temperature on chemistry in protoplanetary disks
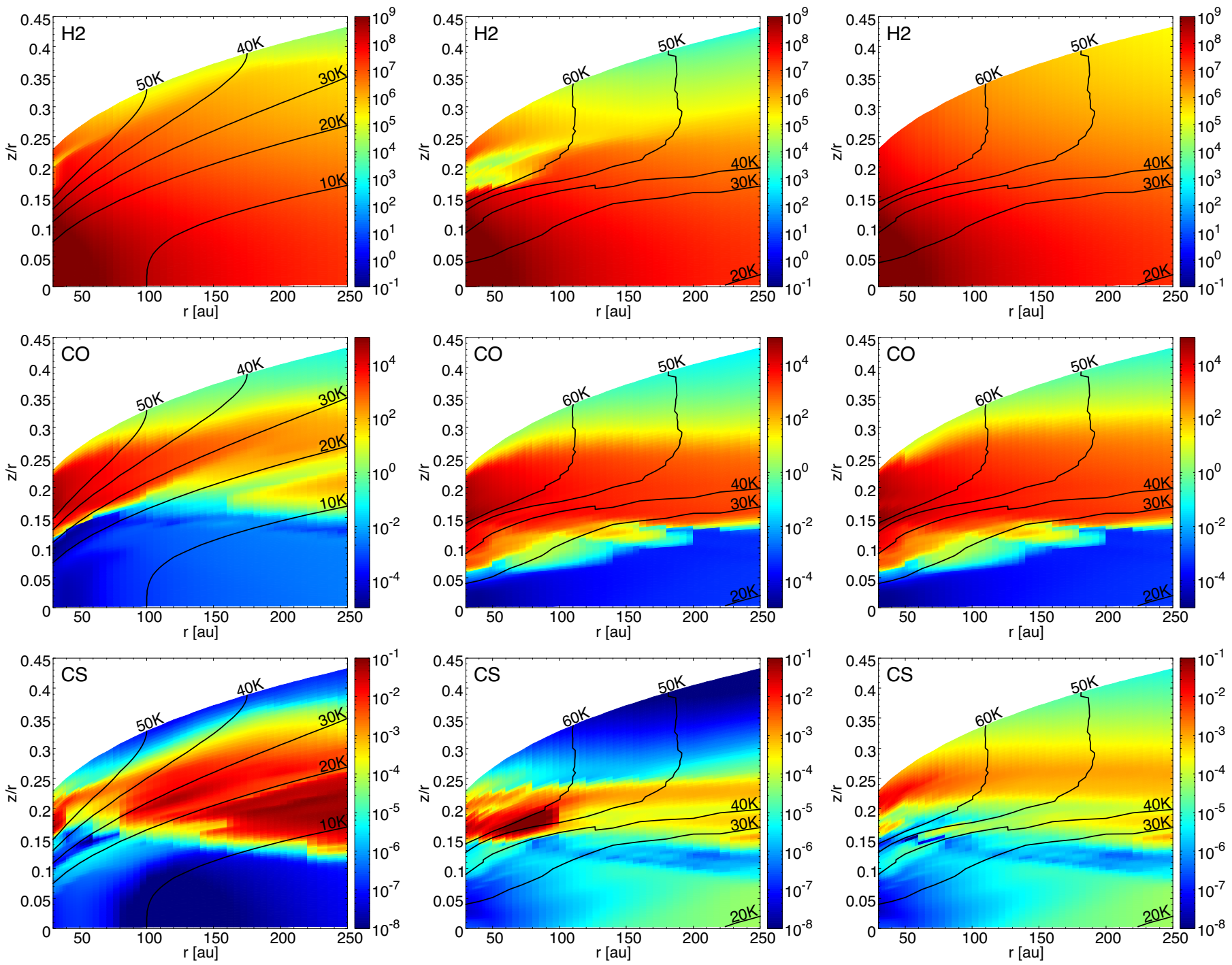

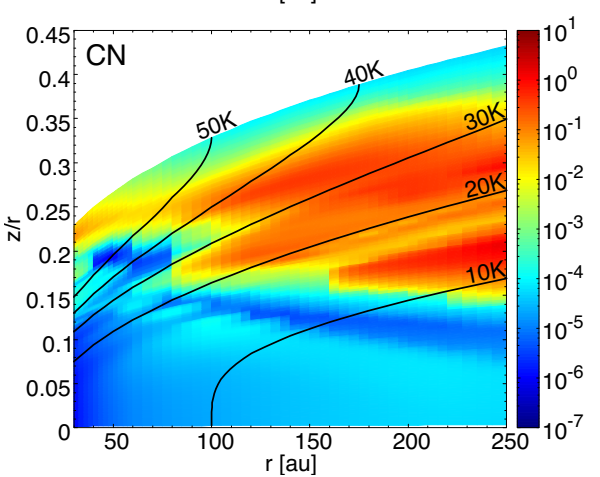

(a) LUV-LH-T

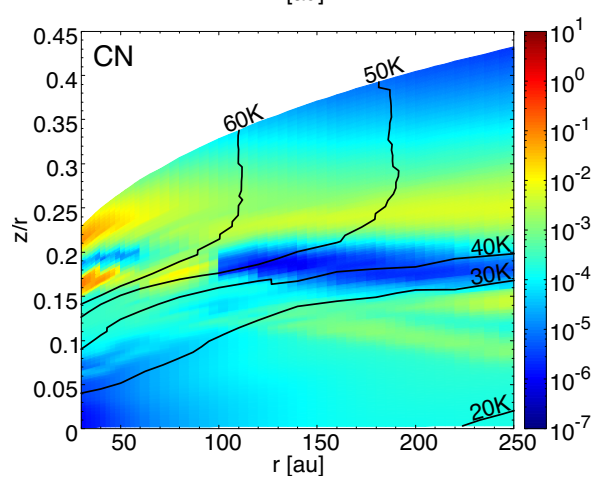

(b) LUV-LH-Ta

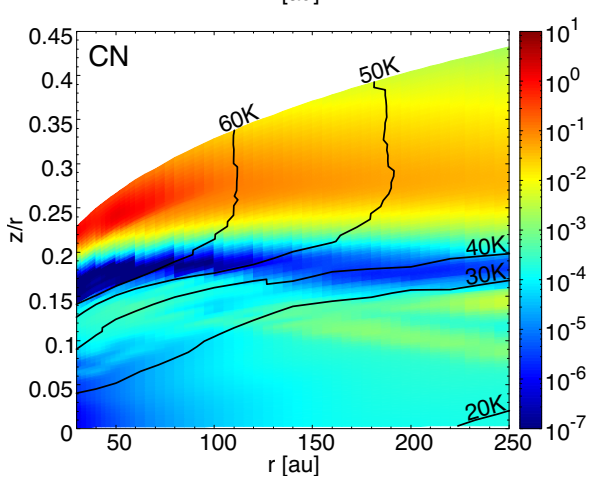

(c) LUV-B14-T

Fig. D.2: Density $\left[\mathrm{cm}^{-3}\right]$ of $\mathrm{H} 2, \mathrm{CO}, \mathrm{CS}$, and $\mathrm{CN}$ in the gas phase of the single-grain models in LUV regime. The left column shows the results of LUV-LH- $T_{\mathrm{g}}$, the middle column indicates the results of LUV-LH- $T_{\mathrm{a}}$, and right column indicates the results of LUV-B14- $T_{\mathrm{a}}$. The black contours represent the dust temperature $\left(\mathrm{T}_{\text {dust }}=\mathrm{T}_{\mathrm{g}}\right.$ in the left column and $\mathrm{T}_{\text {dust }}=\mathrm{T}_{\mathrm{a}}$ in the middle and right columns). 

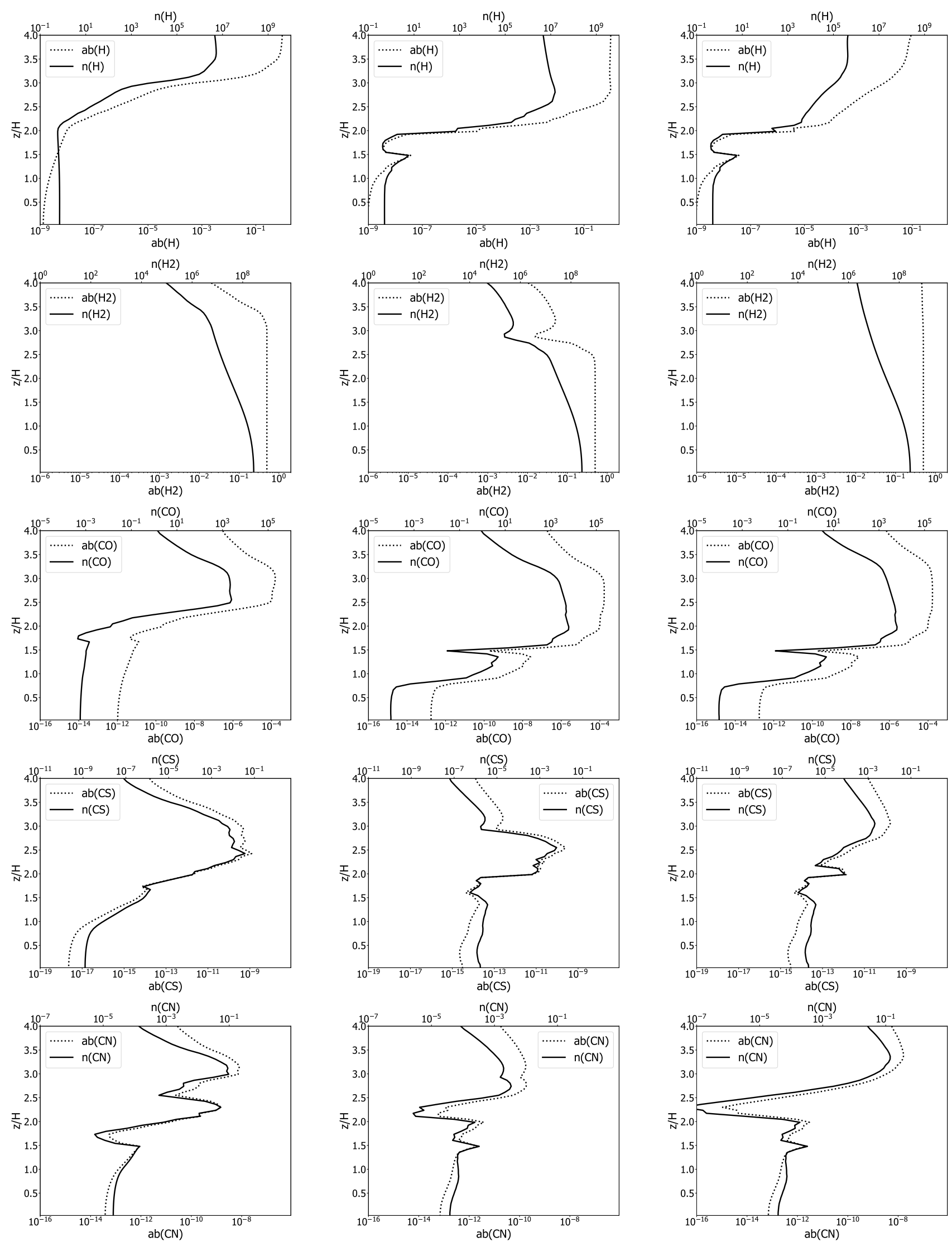

(a) LUV-LH-T $\mathrm{g}_{\mathrm{g}}$

(b) LUV-LH-T

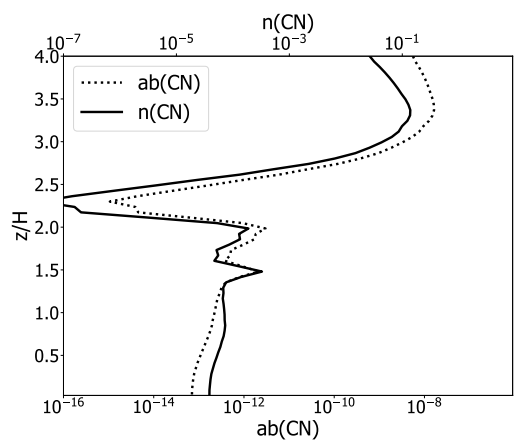

(c) LUV-B14-T

Fig. D.3: Vertical profiles of $\mathrm{H}, \mathrm{H}_{2}, \mathrm{CO}, \mathrm{CS}$, and $\mathrm{CN}$ at 100 au from the star of the LUV single-grain models. The dotted line represents the abundance relative to $\mathrm{H}$ and the solid line shows the density $\left[\mathrm{cm}^{-3}\right]$. 
S. Gavino et al.: Impact of size-dependent grain temperature on chemistry in protoplanetary disks
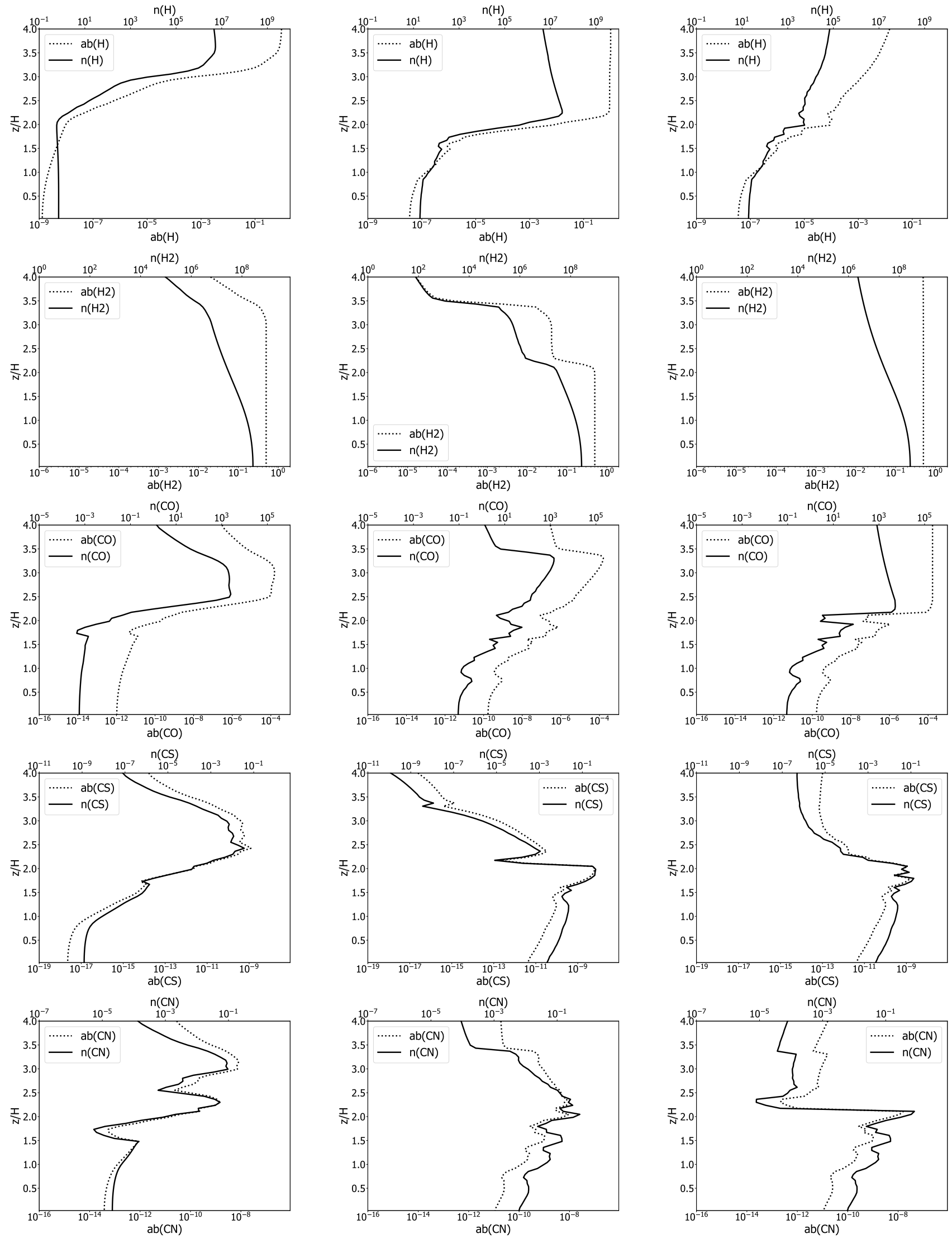

(a) LUV-LH-T

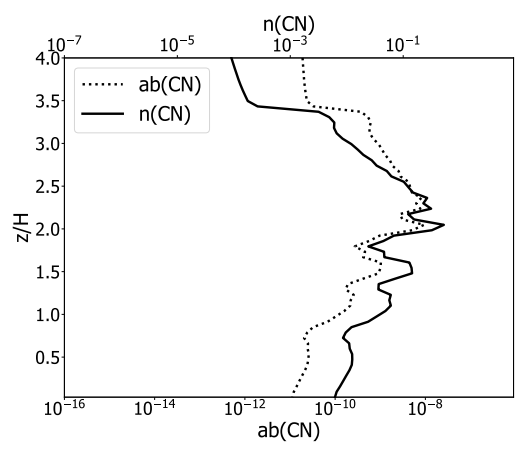

(b) M-LUV-LH

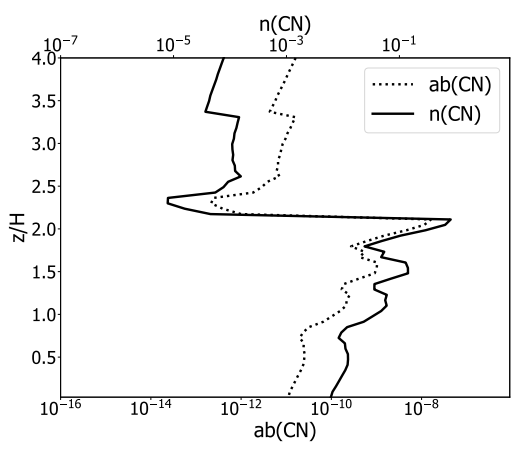

(c) M-LUV-B14

Fig. D.4: Vertical profiles of $\mathrm{H}, \mathrm{H}_{2}, \mathrm{CO}, \mathrm{CS}$, and $\mathrm{CN}$ at 100 au from the star of the LUV single-grain model HUV-LH- $T_{\mathrm{g}}$ in the left column, and multi-grain models in middle and right columns. The dotted line indicates the abundance relative to $\mathrm{H}$ and the solid line indicates the density $\left[\mathrm{cm}^{-3}\right]$. 